\title{
SCORE TESTS FOR TESTING HOMOGENEITY OF RECURRENT EVENT TIMES USING FRAILTY MODELS
}

by

\begin{abstract}
Alia Alkhathami
A thesis submitted to the Faculty of Graduate and Postdoctoral Affairs in partial

fulfilment of the requirements for the degree of

MASTER OF SCIENCE
\end{abstract}

IN

Probability and Statistics

Carleton University

OtTaWA, Ontario

(C) 2015

Alia Alkhathami 


\section{Abstract}

This thesis presents an overview of frailty models in survival analysis for modelling unobserved heterogeneity in survival times. The frailty model is a generalization of Cox's proportional hazard model, where a shared unobserved quantity called frailty describes a positive correlation among the survival times. The frailty term describes the common risks, acting as a factor on the hazard function.

In this thesis, we investigate a score test based on the mixture of chi-square distributions for testing homogeneity of individuals in recurrent event data using a shared frailty model, which is equivalent to testing whether the variance component in a frailty model is zero.

Simulation studies are conducted to assess the empirical level and power of the score test under correctly specified and misspecified random effects, and to study the finite-sample properties in terms of biases and mean squared errors of the estimators under both correctly specified and misspecified frailty models.

The simulation results indicate that when the sample size is small, the empirical levels of the score tests are generally lower than the nominal $5 \%$ level. But they tend to get closer to the nominal level when the sample size is large.

Also, for estimating the model parameters, the ML method appears to provide roughly unbiased estimates of the regression parameters and variance components under correctly specified frailty models. However, under misspecified models, the ML method appears to provide estimators with large biases and mean squared errors.

An application of the score test for frailty variance component is illustrated by using a 
data set of recurrent events of tumours referred to as the bladder cancer data. 


\section{Acknowledgements}

I am greatly indebted to my supervisor, Dr. Sanjoy Sinha, for kindly providing guidance throughout the development of this thesis. His directions and comments have been of the greatest help at all times. This work could not have been done without his guidance.

I extend my sincere thanks to many people in the School of Mathematics and Statistics at Carleton University for their help, continual support, and constructive advice during my graduate education, especially, Abdulaziz Dahir, Harun Kalayci, and Masahiro Oishi.

In addition, I would like to thank my country for supporting me to gain further knowledge in one of the most required field in the development of several sectors. As a return, I intend to dedicate myself in the statistical research and apply them to address real-life issues in my home country.

Finally, my deepest thanks to my father and my whole family, especially, my brothers Abdullah and Mesfer for their love, encouragement, patience and support. 


\section{List of Tables}

2.1 Estimate of survival probability of prognosis for two groups of women with breast cancer trial where censored survival times are labelled with asterisk $(*) .13$

5.1 Empirical level and power of the score test for shared frailty model under correctly specified random effects: $u_{i} \sim N\left(0, \sigma_{u}^{2}\right)$. . . . . . . . . . . 48

5.2 Empirical level and power of the score test for shared frailty model under misspecified random effects: $u_{i}=\sigma_{u}\left(\frac{a_{i}-\lambda}{\sqrt{\lambda}}\right)$, where $a_{i} \sim G a(\lambda, 1)$ and $\lambda=0.5,1.49$

5.3 Empirical level and power of the score test for shared frailty model under misspecified random effects: $u_{i}=\sigma_{u}\left(\frac{a_{i}-\lambda}{\sqrt{\lambda}}\right)$, where $a_{i} \sim G a(\lambda, 1)$ and $\lambda=2,4 . \quad 50$

5.4 Empirical biases and mean squared errors (MSEs) of the estimators of the regression parameters $\left(\beta_{0}, \beta_{1}, \beta_{2}\right)$ under misspecified model with no frailty, when the true model is frailty with $u_{i} \sim N\left(0, \sigma_{u}^{2}\right)$ and $\sigma_{u}^{2}=0,0.5$. Regression parameters are fixed at $\left(\beta_{0}, \beta_{1}, \beta_{2}\right)=(-2.53,0.5,-0.25) \ldots \ldots \ldots$

5.5 Empirical biases and mean squared errors (MSEs) of the estimators of the regression parameters $\left(\beta_{0}, \beta_{1}, \beta_{2}\right)$ under misspecified model with no frailty, when the true model is frailty with $u_{i} \sim N\left(0, \sigma_{u}^{2}\right)$ and $\sigma_{u}^{2}=1,4$. Regression parameters are fixed at $\left(\beta_{0}, \beta_{1}, \beta_{2}\right)=(-2.53,0.5,-0.25) \ldots \ldots \ldots$ 
5.6 Empirical biases and mean squared errors (MSEs) of the estimators of both the regression parameters $\left(\beta_{0}, \beta_{1}, \beta_{2}\right)$ and the frailty variance component $\left(\sigma_{u}^{2}\right)$ under the correct model with frailty, when the random effects are correctly specified: $u_{i} \sim N\left(0, \sigma_{u}^{2}\right)$ where $\sigma_{u}^{2}=0.5$. Regression parameters and variance component are fixed at $\left(\beta_{0}, \beta_{1}, \beta_{2}, \sigma_{u}^{2}\right)=(-2.53,0.5,-0.25,0.5) . \ldots . . \quad$. 56

5.7 Empirical biases and mean squared errors (MSEs) of the estimators of both the regression parameters $\left(\beta_{0}, \beta_{1}, \beta_{2}\right)$ and the frailty variance component $\left(\sigma_{u}^{2}\right)$ under the correct model with frailty, when the random effects are correctly specified: $u_{i} \sim N\left(0, \sigma_{u}^{2}\right)$ where $\sigma_{u}^{2}=1$. Regression parameters and variance component are fixed at $\left(\beta_{0}, \beta_{1}, \beta_{2}, \sigma_{u}^{2}\right)=(-2.53,0.5,-0.25,0.5) \ldots \ldots$

5.8 Empirical biases and mean squared errors (MSEs) of the estimators of both the regression parameters $\left(\beta_{0}, \beta_{1}, \beta_{2}\right)$ and the frailty variance component $\left(\sigma_{u}^{2}\right)$ under the correct model with frailty, when the random effects are correctly specified: $u_{i} \sim N\left(0, \sigma_{u}^{2}\right)$ where $\sigma_{u}^{2}=4$. Regression parameters and variance component are fixed at $\left(\beta_{0}, \beta_{1}, \beta_{2}, \sigma_{u}^{2}\right)=(-2.53,0.5,-0.25,0.5) \ldots . .$.

5.9 Empirical biases and mean squared errors (MSEs) of the estimators of the regression parameters $\left(\beta_{0}, \beta_{1}, \beta_{2}\right)$ under misspecified model with no frailty, when the true model is frailty with $u_{i}=\sigma_{u}\left(\frac{a_{i}-\lambda}{\sqrt{\lambda}}\right)$ and $a_{i} \sim G a(\lambda, 1)$ for $\lambda=0.5, \quad \sigma_{u}^{2}=0,0.05$. Regression parameters are fixed at $\left(\beta_{0}, \beta_{1}, \beta_{2}\right)=$ $(-2.53,0.5,-0.25) \ldots \ldots \ldots \ldots \ldots \ldots$

5.10 Empirical biases and mean squared errors (MSEs) of the estimators of the regression parameters $\left(\beta_{0}, \beta_{1}, \beta_{2}\right)$ under misspecified model with no frailty, when the true model is frailty with $u_{i}=\sigma_{u}\left(\frac{a_{i}-\lambda}{\sqrt{\lambda}}\right)$ and $a_{i} \sim G a(\lambda, 1)$ for $\lambda=0.5, \quad \sigma_{u}^{2}=0.1,0.2$. Regression parameters are fixed $\mathrm{a}\left(\beta_{0}, \beta_{1}, \beta_{2}\right)=$ $(-2.53,0.5,-0.25) \ldots \ldots \ldots \ldots \ldots \ldots$ 
5.11 Empirical biases and mean squared errors (MSEs) of the estimators of the regression parameters $\left(\beta_{0}, \beta_{1}, \beta_{2}\right)$ under misspecified model with no frailty, when the true model is frailty with $u_{i}=\sigma_{u}\left(\frac{a_{i}-\lambda}{\sqrt{\lambda}}\right)$ and $a_{i} \sim G a(\lambda, 1)$ for $\lambda=1, \quad \sigma_{u}^{2}=0,0.05$. Regression parameters are fixed at $\left(\beta_{0}, \beta_{1}, \beta_{2}\right)=$ $(-2.53,0.5,-0.25) \ldots \ldots \ldots \ldots \ldots \ldots$

5.12 Empirical biases and mean squared errors (MSEs) of the estimators of the regression parameters $\left(\beta_{0}, \beta_{1}, \beta_{2}\right)$ under misspecified model with no frailty, when the true model is frailty with $u_{i}=\sigma_{u}\left(\frac{a_{i}-\lambda}{\sqrt{\lambda}}\right)$ and $a_{i} \sim G a(\lambda, 1)$ for $\lambda=1, \quad \sigma_{u}^{2}=0.1,0.2$. Regression parameters are fixed at $\left(\beta_{0}, \beta_{1}, \beta_{2}\right)=$ $(-2.53,0.5,-0.25) \ldots \ldots \ldots \ldots \ldots \ldots$

5.13 Empirical biases and mean squared errors (MSEs) of the estimators of the regression parameters $\left(\beta_{0}, \beta_{1}, \beta_{2}\right)$ under misspecified model with no frailty, when the true model is frailty with $u_{i}=\sigma_{u}\left(\frac{a_{i}-\lambda}{\sqrt{\lambda}}\right)$ and $a_{i} \sim G a(\lambda, 1)$ for $\lambda=2, \quad \sigma_{u}^{2}=0,0.05$. Regression parameters are fixed at $\left(\beta_{0}, \beta_{1}, \beta_{2}\right)=$ $(-2.53,0.5,-0.25) \ldots \ldots \ldots \ldots \ldots \ldots$

5.14 Empirical biases and mean squared errors (MSEs) of the estimators of the regression parameters $\left(\beta_{0}, \beta_{1}, \beta_{2}\right)$ under misspecified model with no frailty, when the true model is frailty with $u_{i}=\sigma_{u}\left(\frac{a_{i}-\lambda}{\sqrt{\lambda}}\right)$ and $a_{i} \sim G a(\lambda, 1)$ for $\lambda=2, \quad \sigma_{u}^{2}=0.1,0.2$. Regression parameters are fixed at $\left(\beta_{0}, \beta_{1}, \beta_{2}\right)=$ $(-2.53,0.5,-0.25) \ldots \ldots \ldots \ldots \ldots \ldots$

5.15 Empirical biases and mean squared errors (MSEs) of the estimators of the regression parameters $\left(\beta_{0}, \beta_{1}, \beta_{2}\right)$ under misspecified model with no frailty, when the true model is frailty with $u_{i}=\sigma_{u}\left(\frac{a_{i}-\lambda}{\sqrt{\lambda}}\right)$ and $a_{i} \sim G a(\lambda, 1)$ for $\lambda=4, \quad \sigma_{u}^{2}=0,0.05$. Regression parameters are fixed at $\left(\beta_{0}, \beta_{1}, \beta_{2}\right)=$ $(-2.53,0.5,-0.25) \ldots \ldots \ldots \ldots \ldots$ 
5.16 Empirical biases and mean squared errors (MSEs) of the estimators of the regression parameters $\left(\beta_{0}, \beta_{1}, \beta_{2}\right)$ under misspecified model with no frailty, when the true model is frailty with $u_{i}=\sigma_{u}\left(\frac{a_{i}-\lambda}{\sqrt{\lambda}}\right)$ and $a_{i} \sim G a(\lambda, 1)$ for $\lambda=4, \quad \sigma_{u}^{2}=0.1,0.2$. Regression parameters are fixed at $\left(\beta_{0}, \beta_{1}, \beta_{2}\right)=$ $(-2.53,0.5,-0.25) \ldots \ldots \ldots \ldots \ldots \ldots$

5.17 Empirical biases and mean squared errors (MSEs) of the estimators of both the regression parameters $\left(\beta_{0}, \beta_{1}, \beta_{2}\right)$ and the frailty variance component $\left(\sigma_{u}^{2}\right)$ under normal frailty model, when true $u_{i}=\sigma_{u}\left(\frac{a_{i}-\lambda}{\sqrt{\lambda}}\right)$, with $a_{i} \sim G a(\lambda, 1)$ and $\left(\lambda, \sigma_{u}^{2}\right)=(0.5,0.5)$. Regression parameters and variance component are fixed at $\left(\beta_{0}, \beta_{1}, \beta_{2}, \sigma_{u}^{2}\right)=(-2.53,0.5,-0.25,0.5) \ldots \ldots \ldots$

5.18 Empirical biases and mean squared errors (MSEs) of the estimators of both the regression parameters $\left(\beta_{0}, \beta_{1}, \beta_{2}\right)$ and the frailty variance component $\left(\sigma_{u}^{2}\right)$ under normal frailty model, when true $u_{i}=\sigma_{u}\left(\frac{a_{i}-\lambda}{\sqrt{\lambda}}\right)$, with $a_{i} \sim G a(\lambda, 1)$ and $\left(\lambda, \sigma_{u}^{2}\right)=(0.5,2)$. Regression parameters and variance component are fixed at $\left(\beta_{0}, \beta_{1}, \beta_{2}, \sigma_{u}^{2}\right)=(-2.53,0.5,-0.25,0.5) \ldots \ldots \ldots$

5.19 Empirical biases and mean squared errors (MSEs) of the estimators of both the regression parameters $\left(\beta_{0}, \beta_{1}, \beta_{2}\right)$ and the frailty variance component $\left(\sigma_{u}^{2}\right)$ under normal frailty model, when true $u_{i}=\sigma_{u}\left(\frac{a_{i}-\lambda}{\sqrt{\lambda}}\right)$, with $a_{i} \sim G a(\lambda, 1) \&$ $\left(\lambda, \sigma_{u}^{2}\right)=(2,2)$. Regression parameters and variance component are fixed at $\left(\beta_{0}, \beta_{1}, \beta_{2}, \sigma_{u}^{2}\right)=(-2.53,0.5,-0.25,0.5) \ldots \ldots \ldots$

6.1 Analysis of bladder cancer data using a standard proportional hazards model without frailty $\left(\sigma_{u}^{2}=0\right) \ldots \ldots \ldots \ldots \ldots$

6.2 Analysis of bladder cancer data using a proportional hazards model with a shared frailty $\left(\sigma_{u}^{2} \neq 0\right) \ldots \ldots \ldots \ldots \ldots$

8.1 Multiple Tumour Recurrence Data for Placebo \& Thiotepa Groups (Wei [30]) 101

8.2 Multiple Tumour Recurrence Data for Placebo \& Thiotepa Groups (Wei [30]) 102 


\section{List of Figures}

2.1 Event and censoring times of subjects in survival analysis. . . . . . . . . . 7

2.2 Graph of survival function $S(t)$ theoretically and in practice. . . . . . . . 9

2.3 Relationships between parametric hazard and survival curves: (a) constant hazard exponential,(b) increasing Weibull, (c) decreasing Weibull, (d) increasing and then decreasing log-normal. . . . . . . . . . . . . . . . 10

2.4 Kaplan-Meier survival curves for two groups of women with breast cancer: $\mathrm{HPA}-\mathrm{vs} . \mathrm{HPA}+\ldots \ldots \ldots \ldots$. . . . . . . . . . . . . . . . 14

2.5 Hazard function for exponential distribution with $\lambda=0.5,1,3 \ldots \ldots$

2.6 Probability density function for exponential distribution with $\lambda=0.5,1,3$. . 18

2.7 Hazard and density functions for Weibull survival times with different values of shape parameter $\gamma=(0.5,1,2,3)$ and scale parameter $\lambda=(1,2) \ldots \ldots$

5.1 Empirical biases and mean squared errors (MSEs) of the estimators of both the regression parameters $\left(\beta_{0}, \beta_{1}, \beta_{2}\right)$ and the frailty variance component $\left(\sigma_{u}^{2}\right)$ under the correct model with frailty, when the random effects are correctly specified: $u_{i} \sim N\left(0, \sigma_{u}^{2}\right)$ where $\sigma_{u}^{2}=0.5$ and the cluster size $(n)=5$. Regression parameters and variance component are fixed at $\left(\beta_{0}, \beta_{1}, \beta_{2}, \sigma_{u}^{2}\right)=$ $(-2.53,0.5,-0.25,0.5) . \ldots \ldots \ldots \ldots$ 
5.2 Empirical biases and mean squared errors (MSEs) of the estimators of both the regression parameters $\left(\beta_{0}, \beta_{1}, \beta_{2}\right)$ and the frailty variance component $\left(\sigma_{u}^{2}\right)$ under the correct model with frailty, when the random effects are correctly specified: $u_{i} \sim N\left(0, \sigma_{u}^{2}\right)$ where $\sigma_{u}^{2}=0.5$ and the cluster size $(n)=10$. Regression parameters and variance component are fixed at $\left(\beta_{0}, \beta_{1}, \beta_{2}, \sigma_{u}^{2}\right)=$ $(-2.53,0.5,-0.25,0.5) . \ldots \ldots \ldots \ldots$

5.3 Empirical biases and mean squared errors (MSEs) of the estimators of both the regression parameters $\left(\beta_{0}, \beta_{1}, \beta_{2}\right)$ and the frailty variance component $\left(\sigma_{u}^{2}\right)$ under the correct model with frailty, when the random effects are correctly specified: $u_{i} \sim N\left(0, \sigma_{u}^{2}\right)$ where $\sigma_{u}^{2}=1$ and the cluster size $(n)=5$. Regression parameters and variance component are fixed at $\left(\beta_{0}, \beta_{1}, \beta_{2}, \sigma_{u}^{2}\right)=$ $(-2.53,0.5,-0.25,0.5) \ldots \ldots \ldots \ldots \ldots$

5.4 Empirical biases and mean squared errors (MSEs) of the estimators of both the regression parameters $\left(\beta_{0}, \beta_{1}, \beta_{2}\right)$ and the frailty variance component $\left(\sigma_{u}^{2}\right)$ under the correct model with frailty, when the random effects are correctly specified: $u_{i} \sim N\left(0, \sigma_{u}^{2}\right)$ where $\sigma_{u}^{2}=1$ and the cluster size $(n)=10$. Regression parameters and variance component are fixed at $\left(\beta_{0}, \beta_{1}, \beta_{2}, \sigma_{u}^{2}\right)=$ $(-2.53,0.5,-0.25,0.5) \ldots \ldots \ldots \ldots \ldots$

5.5 Empirical biases and mean squared errors (MSEs) of the estimators of both the regression parameters $\left(\beta_{0}, \beta_{1}, \beta_{2}\right)$ and the frailty variance component $\left(\sigma_{u}^{2}\right)$ under the correct model with frailty, when the random effects are correctly specified: $u_{i} \sim N\left(0, \sigma_{u}^{2}\right)$ where $\sigma_{u}^{2}=4$ and the cluster size $(n)=5$. Regression parameters and variance component are fixed at $\left(\beta_{0}, \beta_{1}, \beta_{2}, \sigma_{u}^{2}\right)=$ $(-2.53,0.5,-0.25,0.5) \ldots \ldots \ldots \ldots \ldots$ 
5.6 Empirical biases and mean squared errors (MSEs) of the estimators of both the regression parameters $\left(\beta_{0}, \beta_{1}, \beta_{2}\right)$ and the frailty variance component $\left(\sigma_{u}^{2}\right)$ under the correct model with frailty, when the random effects are correctly specified: $u_{i} \sim N\left(0, \sigma_{u}^{2}\right)$ where $\sigma_{u}^{2}=4$ and the cluster size $(n)=10$. Regression parameters and variance component are fixed at $\left(\beta_{0}, \beta_{1}, \beta_{2}, \sigma_{u}^{2}\right)=$ $(-2.53,0.5,-0.25,0.5) \ldots \ldots \ldots \ldots \ldots$

5.7 Empirical biases and mean squared Errors (MSEs) of the estimators of both the regression parameters $\left(\beta_{0}, \beta_{1}, \beta_{2}\right)$ and the frailty variance component $\left(\sigma_{u}^{2}\right)$ under normal frailty model, when true $u_{i}=\sigma_{u}\left(\frac{a_{i}-\lambda}{\sqrt{\lambda}}\right)$, with $a_{i} \sim G a(\lambda, 1)$ and $\left(\lambda, \sigma_{u}^{2}\right)=(0.5,0.5)$ and the cluster size $(n)=5$. Regression parameters and variance component are fixed at $\left(\beta_{0}, \beta_{1}, \beta_{2}, \sigma_{u}^{2}\right)=(-2.53,0.5,-0.25,0.5)$. .

5.8 Empirical biases and mean squared errors (MSEs) of the estimators of both the regression parameters $\left(\beta_{0}, \beta_{1}, \beta_{2}\right)$ and the frailty variance component $\left(\sigma_{u}^{2}\right)$ under normal frailty model, when true $u_{i}=\sigma_{u}\left(\frac{a_{i}-\lambda}{\sqrt{\lambda}}\right)$, with $a_{i} \sim G a(\lambda, 1)$ and $\left(\lambda, \sigma_{u}^{2}\right)=(0.5,0.5)$ and the cluster size $(n)=10$. Regression parameters and variance component are fixed at $\left(\beta_{0}, \beta_{1}, \beta_{2}, \sigma_{u}^{2}\right)=(-2.53,0.5,-0.25,0.5)$.

5.9 Empirical biases and mean squared errors (MSEs) of the estimators of both the regression parameters $\left(\beta_{0}, \beta_{1}, \beta_{2}\right)$ and the frailty variance component $\left(\sigma_{u}^{2}\right)$ under normal frailty model, when true $u_{i}=\sigma_{u}\left(\frac{a_{i}-\lambda}{\sqrt{\lambda}}\right)$, with $a_{i} \sim G a(\lambda, 1)$ and $\left(\lambda, \sigma_{u}^{2}\right)=(0.5,2)$ and the cluster size $(n)=5$. Regression parameters and variance component are fixed at $\left(\beta_{0}, \beta_{1}, \beta_{2}, \sigma_{u}^{2}\right)=(-2.53,0.5,-0.25,0.5)$. . .

5.10 Empirical biases and mean squared errors (MSEs) of the estimators of both the regression parameters $\left(\beta_{0}, \beta_{1}, \beta_{2}\right)$ and the frailty variance component $\left(\sigma_{u}^{2}\right)$ under normal frailty model, when true $u_{i}=\sigma_{u}\left(\frac{a_{i}-\lambda}{\sqrt{\lambda}}\right)$, with $a_{i} \sim G a(\lambda, 1)$ and $\left(\lambda, \sigma_{u}^{2}\right)=(0.5,2)$ and the cluster size $(n)=10$. Regression parameters and variance component are fixed at $\left(\beta_{0}, \beta_{1}, \beta_{2}, \sigma_{u}^{2}\right)=(-2.53,0.5,-0.25,0.5)$. . 
5.11 Empirical biases and mean squared Errors (MSEs) of the estimators of both the regression parameters $\left(\beta_{0}, \beta_{1}, \beta_{2}\right)$ and the frailty variance component $\left(\sigma_{u}^{2}\right)$ under normal frailty model, when true $u_{i}=\sigma_{u}\left(\frac{a_{i}-\lambda}{\sqrt{\lambda}}\right)$, with $a_{i} \sim G a(\lambda, 1)$ and $\left(\lambda, \sigma_{u}^{2}\right)=(2,2)$ and the cluster size $(n)=5$. Regression parameters and variance component are fixed at $\left(\beta_{0}, \beta_{1}, \beta_{2}, \sigma_{u}^{2}\right)=(-2.53,0.5,-0.25,0.5)$. .

5.12 Empirical biases and mean squared errors (MSEs) of the estimators of both the regression parameters $\left(\beta_{0}, \beta_{1}, \beta_{2}\right)$ and the frailty variance component $\left(\sigma_{u}^{2}\right)$ under normal frailty model, when true $u_{i}=\sigma_{u}\left(\frac{a_{i}-\lambda}{\sqrt{\lambda}}\right)$, with $a_{i} \sim G a(\lambda, 1)$ and $\left(\lambda, \sigma_{u}^{2}\right)=(2,2)$ and the cluster size $(n)=10$. Regression parameters and variance component are fixed at $\left(\beta_{0}, \beta_{1}, \beta_{2}, \sigma_{u}^{2}\right)=(-2.53,0.5,-0.25,0.5)$. . 


\section{Contents}

$\begin{array}{ll}\text { Abstract } & \text { i }\end{array}$

$\begin{array}{ll}\text { Acknowledgements } & \text { iii }\end{array}$

List of Abbreviation $\quad$ iv

1 Introduction $\quad 1$

2 Introduction to Survival Analysis $\quad 3$

2.1 Censoring and Truncation $\ldots \ldots \ldots \ldots \ldots \ldots$

2.2 Basic Concepts in Survival Analysis . . . . . . . . . . . . . . . . 8

2.3 Non-parametric and Semi-parametric Models . . . . . . . . . . . . . . . . . 11

2.3.1 Kaplan-Meier Estimator . . . . . . . . . . . . . . . . . . . 11

2.3.2 Cox Proportional Hazards Model . . . . . . . . . . . . . . . . . . . 14

2.4 Parametric Survival Models . . . . . . . . . . . . . . . . . . 16

2.4 .1 Exponential distribution . . . . . . . . . . . . . . 16

2.4 .2 Weibull distribution . . . . . . . . . . . . . . . . . . 18

2.5 Fitting Parametric Survival Models . . . . . . . . . . . . . . . . . . . 20

$2.5 .1 \quad$ Fitting the exponential distribution . . . . . . . . . . . . 22

2.5 .2 Fitting the Weibull distribution . . . . . . . . . . . . . 24

3 Frailty Models for Recurrent Event Data $\quad 27$ 
3.1 Overview of Recurrent Event Data . . . . . . . . . . . . . 27

3.2 Frailty Models . . . . . . . . . . . . . . . . . . . . . . . . . . 28

3.2 .1 Univariate Frailty Models . . . . . . . . . . . . . . . . . . . . 29

$3.2 .2 \quad$ Multivariate Frailty Models . . . . . . . . . . . . . . . . . . . 31

3.2.3 Shared Frailty Models for Recurrent Events . . . . . . . . . . . . . 32

3.2.3.1 The Parametric Proportional Hazards Models (PH) with Shared Frailty . . . . . . . . . . . . . . . 33

3.2.4 Correlated Frailty Models for Recurrent Events . . . . . . . . . . . . 34

4 Score Test 36

4.1 Introduction . . . . . . . . . . . . . . . . 36

4.2 The Score Test . . . . . . . . . . . . . . . . . . . . . 37

4.2 .1 The Model Structure . . . . . . . . . . . . . . . . . . . . 37

$4.2 .2 \quad$ Score Statistics . . . . . . . . . . . . . . . . . . . . 39

4.2.3 Likelihood Function and Parameter Estimation _ . . . . . . . . . 42

$4.2 .3 .1 \quad$ Under No Frailty $\left(\sigma_{u}^{2}=0\right) \ldots \ldots \ldots \ldots \ldots$

$4.2 .3 .2 \quad$ Under Frailty $\left(\sigma_{u}^{2} \neq 0\right) \ldots \ldots \ldots \ldots$

5 Simulation Study 45

5.1 Generated Clustered Data . . . . . . . . . . . . . . . . . 45

5.2 Empirical Level and Power of the Score Test for Shared Frialty . . . . . . . 47

5.2.1 Correctly Specified Random Effects Distribution . . . . . . . . . . 47

5.2 .2 Misspecified Random Effects Distribution _. . . . . . . . . . 48

$5.3 \quad$ Empirical Biases and Mean Squared Errors . . . . . . . . . . . . . . 51

5.3 .1 True Model: Frailty with $u_{i} \sim N\left(0, \sigma_{u}^{2}\right) \ldots \ldots \ldots \ldots 2$

$5.3 .1 .1 \quad$ Fitted Model: No Frailty . . . . . . . . . . . . . . 52

5.3.1.2 Fitted Model: Frailty with $u_{i} \sim N\left(0, \sigma_{u}^{2}\right) \ldots \ldots \ldots 5$

5.3.2 True Model: Frailty with $u_{i}=\sigma_{u}\left(\frac{a_{i}-\lambda}{\sqrt{\lambda}}\right)$ and $a_{i} \sim G a(\lambda, 1) \ldots 63$ 
5.3.2.1 Fitted Model: No Frailty . . . . . . . . . . . . . . 63

5.3.2.2 Fitted Model: Frailty with $u_{i} \sim N\left(0, \sigma_{u}^{2}\right) \ldots \ldots \ldots 7$

6 Application: Bladder Cancer Data $\quad 82$

6.1 Bladder Cancer Study . . . . . . . . . . . . . . . . . . . . . . . 82

6.1 .1 The Model . . . . . . . . . . . . . . . . . . . . . . 83

6.1 .2 Analysis of the Recurrent Events . . . . . . . . . . . . . . 84

7 Conclusion $\quad 86$

$7.1 \quad$ Future Research . . . . . . . . . . . . . . . . . . . . . . . . . . . . 87

$\begin{array}{llr}8 & \text { Appendix } & 88\end{array}$

8.1 Derivation of the Score Test Statistic . . . . . . . . . . . . . 88

8.2 Blader Cancer Data . . . . . . . . . . . . . . . . . . . . . 100

$\begin{array}{ll}\text { Bibliography } & 103\end{array}$ 


\section{Chapter 1}

\section{Introduction}

In the analysis of survival data, it is not frequently the case that survival times can be assumed to be independent. In many applications, multiple outcomes or recurrent times of the same event for a given individual are often considered, especially in clinical trials. In some cases, different groups of individuals share a common feature such as relative or twins. In such data, frailty models can be used for modelling the dependence of survival times or the association between repeated event times. The frailty term is considered to describe the common risks, acting as a factor on the hazard function. Frailties can be used to address the problem of unobserved heterogeneity in event times resulting from different resources. The frailty model is a generalization of Cox's proportional hazard model, where a shared unobserved quantity called frailty describes a positive correlation among the survival times. Frailties are generally assumed to be random variables and follow some distribution with mean zero and unknown variance component which needs to be estimated. In this thesis, we will assume that the frailties $u_{i}$ 's are independent and follow a normal distribution with mean $E\left(u_{i}\right)=0$ and variance $\operatorname{var}\left(u_{i}\right)=\sigma_{u}^{2}$.

In this thesis, we explore a score test based on the mixture of chi-square distributions for testing homogeneity of individuals in clustered survival data, which is equivalent to testing whether the variance component in a frailty model is zero. The use of score test for testing 
frailty variance components is more valid to apply as compared to the likelihood ratio test, because it only requires an estimation of the model parameters under the null hypothesis of no frailty. Also, we investigate the empirical level and power of the score test for a shared frailty model under both correctly specified random effects $\left(i . e ., u_{i} \sim N\left(0, \sigma_{u}^{2}\right)\right)$ and misspecified random effects (i.e., $u_{i}=\sigma_{u}\left(\frac{a_{i}-\lambda}{\sqrt{\lambda}}\right)$, with $a_{i} \sim G a(\lambda, 1)$ ). In addition, we study the finite-sample properties of empirical biases and mean squared errors of the parameter estimates under correctly specified and misspecified random effect distributions. We are interested in studying the impact of misspecification of the random effects assumptions in terms of biases and mean squared errors of the estimators. We also study the impact of misspecified random effects distributions on the empirical level and power of the score test for frailty model.

This thesis is organized as follows. In chapter 2, we briefly review the basic concepts of survival analysis and present definitions of common terminology. In chapter 3 , we introduce the concept of frailty models that are used to describe unobserved heterogeneity for univariate (independent) failure times and multivariate (dependent) failure times. We describe the frailty model as an extension of the proportional hazard $(\mathrm{PH})$ model for analyzing clustered data or repeated measurements. In chapter 4, we describe the use of asymptotic score test based on the mixture of chi-square distributions for testing homogeneity of individuals in recurrent event data using a shared frailty model. Here, we illustrate the calculation of the score statistics from a Taylor series expansion of the likelihood function about $E_{u_{i}}\left(u_{i}\right)=0$. In chapter 5, we ran a series of simulations to investigate the biases and mean squared errors of parameter estimates under both correctly specified and misspecified random effects distributions. An illustrative example of the score test is discussed using recurrent event time data obtained from a clinical trial in chapter 6. Finally, concluding remarks with some directions for future research are made in Chapter 7. In the appendix section, we provide details about the data used in this thesis and mathematical derivations of the score test. 


\section{Chapter 2}

\section{Introduction to Survival Analysis}

Survival analysis is one of the most important statistical techniques that have been used in many research areas such as medicine, economics, engineering, epidemiology, demography, and other fields. Methods for survival analysis were developed for the purpose of followingup individuals or subjects through time from the onset of risk or exposure of an event until the occurrence of that event. In other words, we follow up the changes in cases over time and examine any experience of a particular event of interest. Survival analysis is not only used in randomized clinical trials, for example, to compare the occurrence of an outcome event after patients are given different treatments, but survival models are also used to test the presence of any epidemiological association in observational research. However, the use of survival analysis is not limited to medical and epidemiological studies but it is also being used to analyze non-medical data as we will see in the following discussion. To begin with, it is reasonable to discuss certain questions such as: What distinguishes survival analysis from other fields in statistics? Why do we use survival analysis to deal with special data in certain research areas? What is the structure of survival data and why does this kind of data need a special statistical theory? Why are different survival techniques being used? In the following lines we will explain clearly those considerations of using survival analysis for statistical purposes. 
In clinical research, simple forms of analysis relying on fixed time periods can be used to study the likelihood of an event in a group of patients. Investigators might calculate the risk of experiencing an event by measuring the proportion of patients who suffer this particular event during a fixed period of time. However, this simple approach has several problems that need to be considered. For instance, some patients will be known to be alive at the end of trial, others will be known to be dead, but certain patients will be lost to follow up for particular reasons. For example, some might have moved to another place, others did not experience the event during this period, while some may decide to leave prior to the end of study, or they might suffer another event which causes them to be deceased, which are referred to as censored observations, as will be discussed later. In these cases, this simple approach of analysis is not valid because patients whose status regarding the event is unknown will be excluded even though they have been followed for a couple of weeks or months. Another problem is that some patients tend to be followed-up for a longer period than others in medical research, so those patients who are enrolled in the early stages of a trial and followed up for a short period would have to be excluded from the analysis due to insufficient follow-up time. In addition, patients who experience the event of interest (e.g., death or another event) after two weeks will have an equal weighting in the analysis as those who experience the same event after 30 weeks, whereas if the event occurs for others 60 weeks after the end of the study, their event status will not be included in the analysis. Therefore, survival analysis is used to take into account these different follow-up times.

The main difference between survival analysis and other types of analysis is that it deals with "censored data". In survival analysis, censored observations for some individuals or subjects in the study which provide only partial information about the random variable (time) will be taken into account in such analysis. As a consequence of censoring, survival times for individuals or subjects are a mixture of both discrete (censoring indicator) and continuous (event/censoring time) data that need a different type of analysis than that used in the traditional discrete and continuous case (see details in Winenke [31]). 
Another piece of information in survival analysis is that the outcome variable of interest is referred to as the time variable and is often called a survival time, follow-up time, lifetime, event time, time to event, failure time, event-history, and duration time as synonyms. This time (response variable) is an interval that could be in years, months, weeks, or days from the beginning of follow-up of an individual until an event of interest or censoring occurs. We consider a random variable $T$ as the event time, which is usually assumed to be nonnegative, and assumed to follow a continuous distribution so that all functions of time-toevent distribution are over the interval $[0, \infty)$, and we will mainly focus on this case of analysis in this thesis. The term "survival time" is not only used with death as an event, but is also used with other events in several fields such as disease, incidence, complications from surgery, relapse or recovery (e.g., return to work) in medical fields. In engineering, the event could be the failure of a technical component. In demography, the event could be death, leaving the house, pregnancy, divorce, or marriage. In economics, the event can be employment, retirement, or repaying bank loans. In political science, events such as international conflicts, elections, or cabinet transitions are considered. In crimes, arrest or criminal recidivism are great events of interest in preventing crimes or making decisions on the efficiency of punishments. By event, we mean any designated experience of interest that might happens to an individual and need to be investigated for making efficient conclusions.

\subsection{Censoring and Truncation}

Censoring is the particular feature that distinguishes survival data from other data because the survival times for individuals or subjects are not always observed. In essence, censoring occurs when we have partial information about an individual survival time, and we do not know the exact survival time. That means that individuals or subjects are followed-up for some time, but the event of interest does not occur during this period. In this case, the only thing that is known is that the true survival time exceeds the observed censoring time. 
In general, there are four reasons that might cause censoring to occur:

(i) Lost to follow-up: when an individual is lost to follow-up because the person might move out or emigrate elsewhere so that she or he is never seen again.

(ii) Drop out: when an individual withdraws and decides not to continue the treatment prior to the end of the study or another reason (e.g., leaving the study because of a side effect of a treatment).

(iii) Termination of study: this type of censoring is called administrative censoring. It occurs when the study ends at predefined period of time and the individual does not experience the event before this period.

(iv) Competing risks: when the event of interest can not be observed because of the occurrence of a competing event (e.g., death by adverse drug reaction or by accident).

Survival data (or observations) can be right-censored, left-censored, or interval-censored. Right censoring occurs when the true survival time is equal to or greater than the observed survival time. That means the interval survival time will be incomplete at the right side of the follow-up period. For instance, the individual is lost to follow-up, he or she withdraws from the study, or the study ends without the individual experiencing the event of interest. All these kinds of data are referred to as right-censored since the complete survival time interval, which is not known, will be cut off at the right side of the observed survival time interval. Most survival data are right censored and in this thesis we will consider this kind of censoring. Left-censoring occurs when an individual's survival time is less than the observed survival time. In this case, the event of interest has already occurred before entering the study, but it is not known exactly when. Thus the survival time is censored on the left side since the true survival time is shorter than the follow-up time. For example, the time at which a teenager begins to drink alcohol or the time of infection with a sexually transmitted disease such as HIV/AIDS. Survival data can also be interval-censored, which occurs when a person's 
true (but unobserved) survival time is within a certain known specified time interval. For instance, considering regular testing for infection with HIV/AIDS, a person is found HIV negative at the time $\left(t_{1}\right)$ of the first test and HIV positive at the time $\left(t_{2}\right)$ of the second test. In such a case, this person's true event time occurred after time $\left(t_{1}\right)$ and before time $\left(t_{2}\right)$, so the subject is interval-censored in the time interval $\left(t_{1}, t_{2}\right)$.

In Figure 2.1, the event times of subjects 1, 2, and 7 are completely observed, while the event times of subjects 5 and 3 are censored because of loss to follow-up, drop out, or competing risks. For each of subjects 4 and 6 , the event times are censored because of termination of the study. All of these censored observations are right-censored because the true survival times are not complete at the right side of the follow-up period.

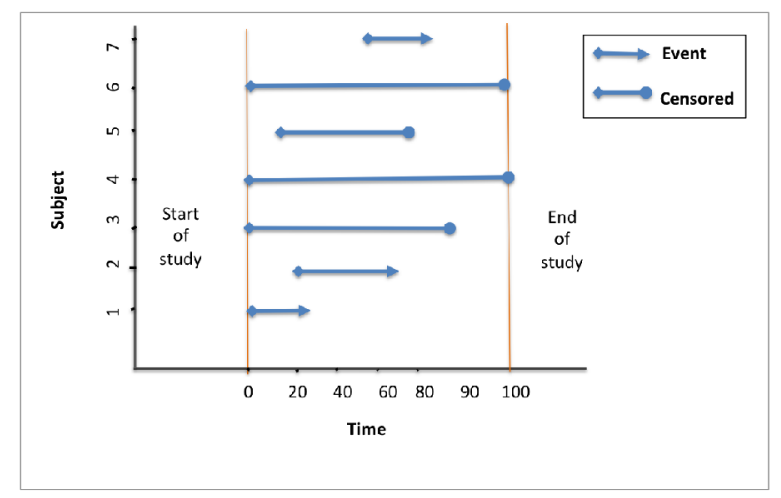

Figure 2.1: Event and censoring times of subjects in survival analysis.

There are three assumptions about censoring often considered for survival data: independent censoring, random censoring, and non-informative censoring (see more details in Kleinbaum and Klein [20]). In this thesis, we consider the assumption of noninformative censoring which depends on the distribution of the time-to-event random variable and the distribution of the time-to-censoring random variable; that is, the censoring is caused by something that is independent of the impending failure, for example, when a subject plans for termination of follow-up or moves out of the city for reasons unrelated to the risk of the event. 
In addition, some survival studies may contain truncation, namely left-truncation, righttruncation, or interval-truncation, which is a variant of censoring which occurs when the incomplete nature of the observation is due to a systematic selection process inherent to the study design. Left-truncated data occur when an unknown subset of individuals or subjects failed before a certain time so that they cannot get in the study due to the structure of the study design. For example, one might study how long individuals that have been hospitalized for a heart attack survive by taking some drug at home, so only those who survive in the hospital will be included in the study. Right-truncated data occur when only the individuals who have experienced the event of interest are observable. More examples and details of truncated data in survival analysis are given in Harrell [16], Kleinbaum and Klein [20], and Wienke [31].

\subsection{Basic Concepts in Survival Analysis}

This section describes basic concepts of univariate survival data and contains mathematical terminology and notation that build the basis for specific points in later chapters. In survival analysis, we consider $T$ as a single random variable for an individual 's survival time, called the response variable, where its values are positive numbers (i.e., $T \geq 0$ ). Also, let $t$ denote

any specific positive value of interest for the random variable $T$. Finally, let $\delta$ the censoring inductor, where $\delta=1$ if the event occurs during the study period and $\delta=0$ if the survival time is censored by the end of the study period.

In survival analysis, the distribution of a random variable $T$ (response variable) is completely and uniquely described by several functions used in specific situations. One of those functions is the survival function, $S(t)$, which gives the probability that a person survives longer than some specified time $t$ given by

$$
S(t)=1-F(t)=\mathrm{P}(T \geq t)=\int_{t}^{\infty} f(s) d s
$$


where $F(t)$ is the cumulative distribution function of the survival time.

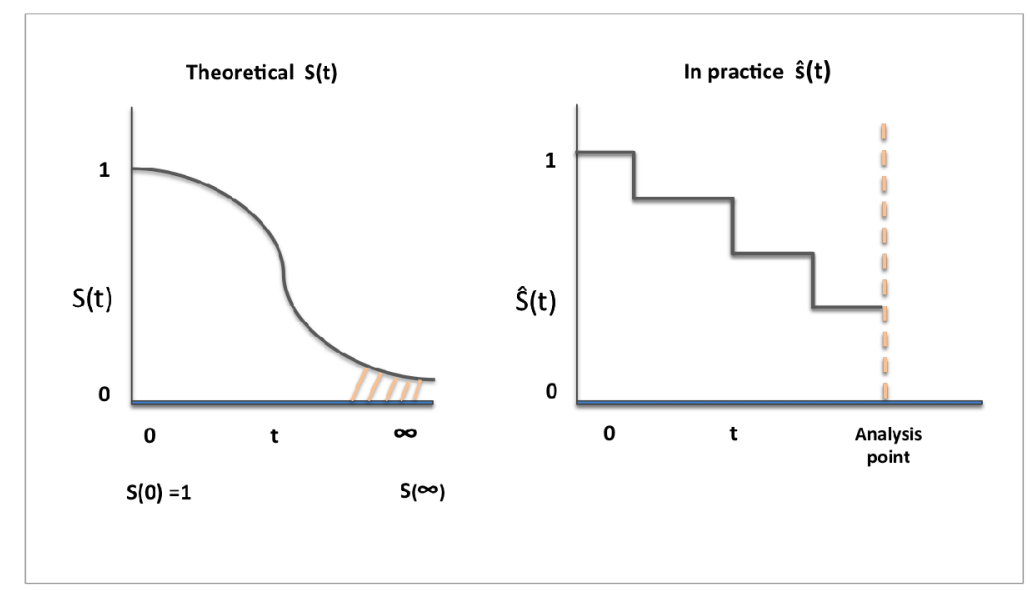

Figure 2.2: Graph of survival function $S(t)$ theoretically and in practice.

Theoretically, as $t$ ranges from 0 to $\infty$, the survival function is graphed as a smooth curve, while in practice we usually obtain graphs that are step functions when using actual data as illustrated in Figure 2.2. Additionally, all survival functions must be non-increasing as $t$ increases; $\mathrm{S}(\mathrm{t})$ is always 1 at $t=0$ and 0 at $t=\infty$.

Another important function in survival analysis is the hazard function, $h(t)$, also called the force of mortality, hazard or failure rate, mortality rate, or incidence rate, which is defined by

$$
h(t)=\lim _{\Delta t \rightarrow 0} \frac{P(t<T \leq t+\Delta t \mid T>t)}{\Delta t}=\frac{f(t)}{S(t)},
$$

where $f(t)$ is the probability density function of $T$ evaluated at t. As illustrated by the definition, the hazard at time $t$ is related to the probability that the event will occur in a small interval around $t$, given that the event has not occurred before time $t$. The hazard function can also be expressed as the negative of the slope of the log of the survival function, that is,

$$
h(t)=-\frac{\partial \log S(t)}{\partial t}
$$


So the integral of $h(t)$ is

$$
\int_{0}^{t} h(v) d v=-\log S(t),
$$

where the integral or area under $h(t)$ is defined to be the cumulative hazard function $H(t)$, given by

$$
H(t)=-\log S(t),
$$

or

$$
S(t)=\exp [-H(t)] .
$$

Therefore, if we know any one of the functions $S(t), H(t), h(t)$, we can derive the other two functions, and these three functions are different ways of describing the same distribution of the response variable $T$.
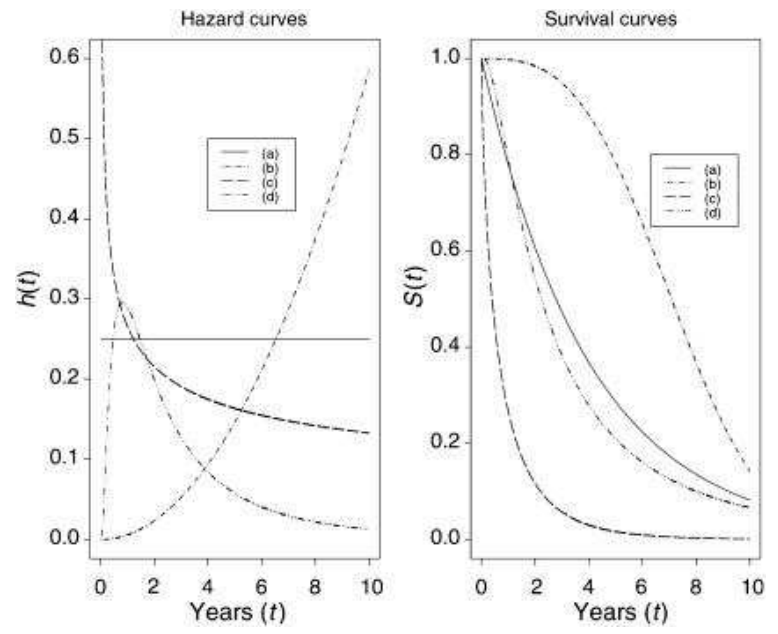

Figure 2.3: Relationships between parametric hazard and survival curves: (a) constant hazard exponential,(b) increasing Weibull, (c) decreasing Weibull, (d) increasing and then decreasing log-normal.

Figure 2.3 shows examples of different shapes for hazard rate and the relationship between those parametrically specified hazards and the corresponding survival probabilities. It illustrates a constant hazard function over time when survival times form an exponential distribution, an increasing or decreasing hazard rates based on a Weibull model, and a 
combination of decreasing and increasing hazard rates using a log-Normal model.

\subsection{Non-parametric and Semi-parametric Models}

The techniques used for dealing with censored data can be broadly classified into nonparametric (Kaplan-Meier product limit method), parametric (Weibull and exponential methods) and semi-parametric method (Cox-proportional hazards method).

\subsubsection{Kaplan-Meier Estimator}

The Kaplan-Meier product limit method (KM) is used when no assumption is made about the functional distribution of the hazard rate with time. For many analyses, this is the main step to select a statistical model for more in-depth analysis, while in others this step might be the last step. In case no event times are censored, a nonparametric estimator of the survival function $S(t)$, defined in equation (2.1), is $1-\hat{F}(t)$ where $\hat{F}(t)$ is the empirical cumulative distribution function that depends on the observed failure times $t_{1}, t_{2}, \ldots, t_{n}$.

Thus the empirical survival function, $\hat{S}(t)$, is given by the fraction of the observed failure times that are equal to or greater than time $t$

$$
\hat{S}(t)=-\frac{\text { (number of } \left.T_{i} \geq t\right)}{n}
$$

When there are censored event times, the survival function, $\mathrm{S}(\mathrm{t})$, can be estimated by the Kaplan-Meier product limit estimator (KM), which is based on conditional probabilities, given by

$$
\hat{S}(t)=\prod_{t_{i}<t}\left(1-\frac{d_{i}}{n_{i}}\right),
$$

where $t_{1}, \ldots, t_{n}$ denote the ordered event times, $d_{i}$ is the number of failure at $t_{i}$, and $n_{i}$ denotes the number of subjects at risk at time $t_{i}$. The Kaplan-Meier estimator of $H(t)$ is $\hat{H}(t)=-\log \hat{S}(t)$ (see Kaplan and Meier [19]). 
A closely related estimator of a survival function is given by $\hat{S}(t)=\exp \{-\hat{H}(t)\}$, where $\hat{H}(t)$ is the Nelson-Aalen estimator of the cumulative hazard function of the form

$$
\hat{H}(t)=\sum_{t_{i} \leq t} \frac{d_{i}}{n_{i}},
$$

(Nelson[23]; Aalen [1]).

To demonstrate the computation of $\hat{S}(t)$, Table 2.1 illustrates an example of calculating Kaplan-Meier survival probability of a sample of survival times in months for two groups of women who had received surgical treatment for breast cancer. The tumours of these women were treated with HPA (Helix Pomatia Agglutinin) and each tumour was classified according to whether it was positively or negatively stained. The objective of an analysis of this retrospective study was to investigate the difference in the survival experience of the two groups of women positively staining and negative staining with HPA. Figure 2.4 shows KM survival curves for this clinical trial, which indicates that the group with negative HPA staining are more likely to live longer after surgery than the other group with positive staining (see Harrell [16] for more details of the survival times data for this breast cancer study). 
Table 2.1: Estimate of survival probability of prognosis for two groups of women with breast cancer trial where censored survival times are labelled with asterisk $(*)$.

\begin{tabular}{|c|c|c|c|c|c|}
\hline \multicolumn{2}{|c|}{ Negative staining $(\mathrm{n}=13)$} & \multicolumn{4}{|c|}{ Positive staining $(\mathrm{n}=32)$} \\
\hline $\begin{array}{l}\text { Survival } \\
\text { time }\end{array}$ & $\begin{array}{c}\text { Kaplan - Meier } \\
\hat{S}(t)\end{array}$ & $\begin{array}{c}\text { Survival } \\
\text { time }\end{array}$ & $\begin{array}{c}\text { Kaplan - Meier } \\
\hat{S}(t)\end{array}$ & $\begin{array}{r}\text { Survival } \\
\text { time }\end{array}$ & $\begin{array}{c}\text { Kaplan - Meier } \\
\hat{S}(t)\end{array}$ \\
\hline 23 & $1 \times(1-1 / 13)=0.923$ & 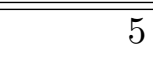 & $1 \times(1-1 / 32)=0.969$ & 68 & $\mathrm{~S}(61) \times(1-1 / 16)=0.469$ \\
\hline 47 & $\mathrm{~S}(23) \times(1-1 / 12)=0.846$ & 8 & $\mathrm{~S}(5) \times(1-1 / 31)=0.938$ & 71 & $\mathrm{~S}(68) \times(1-1 / 15)=0.438$ \\
\hline 69 & $\mathrm{~S}(47) \times(1-1 / 11)=0.769$ & 10 & $\mathrm{~S}(8) \times(1-1 / 30)=0.906$ & $76^{*}$ & $\mathrm{~S}(71) \times(1-0 / 14)=0.438$ \\
\hline $70^{*}$ & $\mathrm{~S}(69) \times(1-0 / 10)=0.769$ & 13 & $\mathrm{~S}(10) \times(1-1 / 29)=0.875$ & $105^{*}$ & $\mathrm{~S}(76) \times(1-0 / 13)=0.438$ \\
\hline $71^{*}$ & $\mathrm{~S}(70) \times(1-0 / 9)=0.769$ & 18 & $\mathrm{~S}(13) \times(1-1 / 28)=0.844$ & $107^{*}$ & $\mathrm{~S}(105) \times(1-0 / 12)=0.438$ \\
\hline $100^{*}$ & $\mathrm{~S}(71) \times(1-0 / 8)=0.769$ & 24 & $\mathrm{~S}(18) \times(1-1 / 27)=0.813$ & $109^{*}$ & $\mathrm{~S}(107) \times(1-0 / 11)=0.438$ \\
\hline $101^{*}$ & $S(100) \times(1-0 / 7)=0.769$ & 26 & $\mathrm{~S}(24) \times(1-1 / 26)=0.781$ & 113 & $\mathrm{~S}(109) \times(1-1 / 10)=0.394$ \\
\hline 148 & $\mathrm{~S}(101) \times(1-1 / 6)=0.640$ & 26 & $\mathrm{~S}(26) \times(1-1 / 25)=0.75$ & $116^{*}$ & $\mathrm{~S}(113) \times(1-0 / 9)=0.394$ \\
\hline 181 & $\mathrm{~S}(148) \times(1-1 / 5)=0.512$ & 31 & $\mathrm{~S}(26) \times(1-1 / 24)=0.719$ & 118 & $\mathrm{~S}(116) \times(1-1 / 8)=0.345$ \\
\hline $198^{*}$ & $\mathrm{~S}(181) \times 1-0 / 4)=0.512$ & 35 & $\mathrm{~S}(31) \times(1-1 / 23)=0.688$ & 143 & $\mathrm{~S}(118) \times(1-1 / 7)=0.295$ \\
\hline $208^{*}$ & $\mathrm{~S}(198) \times(1-0 / 3)=0.512$ & 40 & $\mathrm{~S}(35) \times(1-1 / 22)=0.656$ & $154^{*}$ & $\mathrm{~S}(143) \times(1-0 / 6)=0.295$ \\
\hline $212^{*}$ & $\mathrm{~S}(208) \times(1-0 / 2)=0.512$ & 41 & $\mathrm{~S}(40) \times(1-1 / 21)=0.625$ & $162^{*}$ & $\mathrm{~S}(154) \times(1-0 / 5)=0.295$ \\
\hline $224^{*}$ & $\mathrm{~S}(212) \times(1-0 / 1)=0.512$ & 48 & $\mathrm{~S}(41) \times(1-1 / 20)=0.594$ & $188^{*}$ & $\mathrm{~S}(162) \times(1-0 / 4)=0.295$ \\
\hline & & 50 & $\mathrm{~S}(48) \times(1-1 / 19)=0.563$ & $212^{*}$ & $\mathrm{~S}(188) \times(1-0 / 3)=0.295$ \\
\hline & & 59 & $\mathrm{~S}(50) \times(1-1 / 18)=0.531$ & $217^{*}$ & $\mathrm{~S}(212) \times(1-0 / 2)=0.295$ \\
\hline & & 61 & $\mathrm{~S}(59) \times(1-1 / 17)=0.5$ & $225^{*}$ & $\mathrm{~S}(217) \times(1-0 / 1)=0.295$ \\
\hline
\end{tabular}




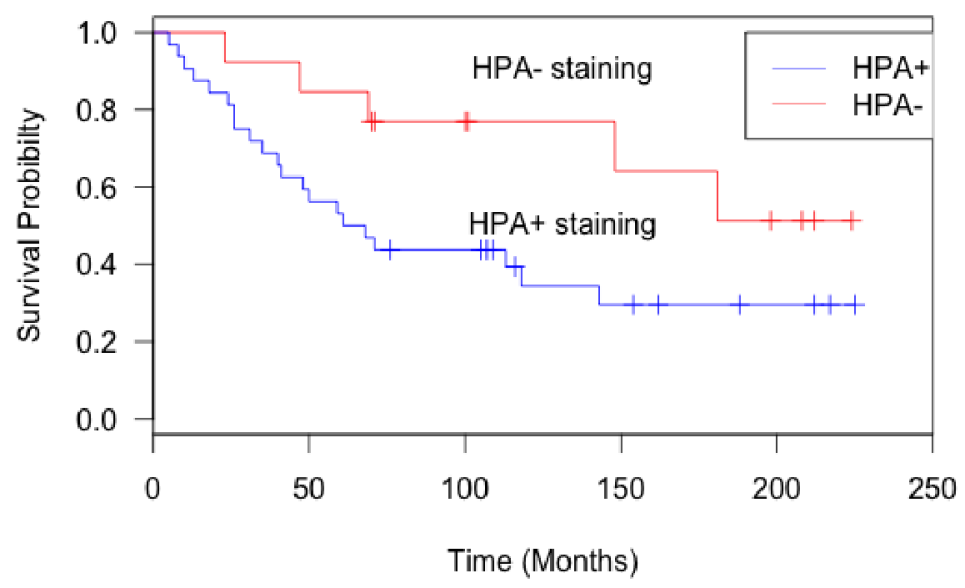

Figure 2.4: Kaplan-Meier survival curves for two groups of women with breast cancer: HPAvs. HPA+ .

In the case of nonparametric comparisons for right-censored data, Kaplan-Meier curves for two or more groups can be evaluated to determine whether or not the groups are equivalent by using the long-rank test (Mantel 1996). The long-rank test is a large-sample chisquare test, which is used to provide an overall comparison of KM curves where the null hypothesis is that all survival curves are the same. Other nonparametric test procedures include the weighted log-rank tests as described in Harrington and Fleming [15]. For more detials about this non-parametric method in survival analysis, see in Kleinbaum and Klein [20] and Harrell [16].

\subsubsection{Cox Proportional Hazards Model}

The non-parametric methods described briefly in the previous section can be suitable for the analysis of a single sample of survival data or for the comparison of survival times between two or more groups. However, in many clinical studies additional information related to each patient, for example, exposure to different medicines (placebo vs. active) or other probabilistically important factors such as sex or age need to be recorded at the outset of 
the study. These risk factors are referred to as explanatory variables, independent variables, or predictor variables, and they can be fixed variables or they may change over time, in which case, they are called time-dependent variables. In such medical studies, it is of great interest for clinicians and biostatisticians to explore the relationship between the survival experience of patients and one or more explanatory variables. In other words, they are interested in investigating whether or not those predictor variables have an impact on the time that the patient survives. The basic model for such survival data is called the proportional hazards model (PH), which was proposed by Cox (1972), and it has also come to be known as the Cox regression model.

This survival model is often used to describe the hazard function at a particular time that depends on $p$ explanatory variables represented by the vector $\boldsymbol{x}_{i}=\left(x_{1}, \ldots, x_{p}\right)^{\prime}$. In particular, it allows the baseline hazard function, $h_{0}(t)$, for an individual for whom the values of the vector $\boldsymbol{x}_{i}$ of all explanatory variables are zero to be multiplied by $\exp \left(\boldsymbol{x}_{i}^{\prime} \boldsymbol{\beta}\right)$, which is a function of the vector of explanatory variables for the $i^{\text {th }}$ individual called the relative hazard.

Therefore, the hazard function for the $i^{\text {th }}$ individual using the proportional hazards model can be written as

$$
h_{i}(t)=h_{0}(t) \exp \left(\boldsymbol{x}_{i}^{\prime} \boldsymbol{\beta}\right)
$$

This model can be re-expressed in the form

$$
\log \left\{\frac{h_{i}(t)}{h_{0}(t)}\right\}=\beta_{1} x_{1 i}+\beta_{2} x_{2 i}+\ldots+\beta_{p} x_{p i}
$$

and for this reason the proportional hazards model may also be regarded as a linear model for the logarithm of the hazard ratio. This $\mathrm{PH}$ model is referred to as a semi-parametric model when no particular form of probability distribution is assumed for the survival times when the baseline hazard model is non-parametrically specified . 


\subsection{Parametric Survival Models}

Parametric survival models are regression models in which the distribution of the response variable (time-to-failure) should have positive support such as the exponential, Weibull, lognormal, log-logistic, Gompertz, but such models also differ from standard regression models in their ability to account for censoring and truncation. As discussed earlier, there is no need to assume a particular form of probability distribution for the survival times when we use the Cox regression model in the analysis of survival data, when the hazard function is not restricted to a specific functional form, and no attempt is made to describe this numerically. However, in survival analysis the assumption of a particular distribution of event times is useful to make more precise inferences such as estimates of relative hazards, the mean, and the median of the survival times. Models in which the survival times are parametrically specified are known as parametric survival models such as the proportional hazards model, accelerated failure time model, and the proportional odds model. In parametric proportional hazards models, we assume a particular parametric function for the baseline hazard $h_{0}(t)$ that leads the event times following the same distribution.

Proportional hazards models based on Weibull distribution are the most widely used in the parametric analysis of survival data. In addition, certain parametric models have been used repeatedly in survival analysis such as exponential, Gompertz, log-logistics, lognormal, and gamma (Wienke [31]). Below we briefly discuss the properties and theoretical basis of both exponential and Weibull distributions in the simplest case, where identically and independently distributed random variable $T$ denotes the time of the occurrence of an event.

\subsubsection{Exponential distribution}

The exponential model $(T \sim \exp (\lambda))$ is the simplest parametric model and assumes that the hazard function is a constant over time, which means the hazard of an event is the same at 
any time after the time origin of the study. This model has only one parameter, which is a positive value that can be estimated by fitting the model to the observed data. In general, once the distributional model for event times is specified in terms of the probability density function (p.d.f), $f(t)$, the corresponding survival and hazard function can be easily obtained from the relationships discussed in section (2.2). Therefore, after some algebra, the following important formulas for exponential distribution can be derived

$$
\begin{gathered}
\text { probability density function, } f(t)=\lambda e^{-\lambda t} \\
\text { survival function, } S(t)=e^{-\lambda t} \\
\text { hazard function, } \quad h(t)=\lambda \\
\text { cumulative hazard function, } H(t)=\lambda t \\
\text { mean, } E(T)=1 / \lambda \\
\text { variance, } \quad V(T)=1 / \lambda^{2}
\end{gathered}
$$

Thus using the proportional hazards $(\mathrm{PH})$ model, the exponential survival regression model can be expressed as:

$$
\begin{aligned}
h(t \mid \boldsymbol{x}) & =\lambda \exp \left(\boldsymbol{x}^{\prime} \boldsymbol{\beta}\right) \\
S(t \mid \boldsymbol{x}) & =\exp \left[-\lambda t \exp \left(\boldsymbol{x}^{\prime} \boldsymbol{\beta}\right)\right] \\
E(t \mid \boldsymbol{x}) & =1 / \lambda \exp \left(\boldsymbol{x}^{\prime} \boldsymbol{\beta}\right) .
\end{aligned}
$$

Since model $(2.10)$ can be written as $h(t \mid \boldsymbol{x})=\exp \left[(\log \lambda)+\boldsymbol{x}^{\prime} \boldsymbol{\beta}\right]$, the parameter $\lambda$ can be thought of as the antilog of an intercept term.

Above exponential model can be re-expressed as $h(t \mid \boldsymbol{x})=\exp \left(\boldsymbol{x}^{\prime} \boldsymbol{\beta}\right)$ if we replace $\lambda$ parameter with an intercept term in $\boldsymbol{x}^{\prime} \boldsymbol{\beta}$, that is, when $\lambda=\exp \left(\beta_{0}\right)$.

A plot of the hazard function for different values of $\lambda$, namely $0.5,1,3$ is given in Figure 2.5, and the corresponding probability density function (p.d.f) are shown in Figure 2.6. 


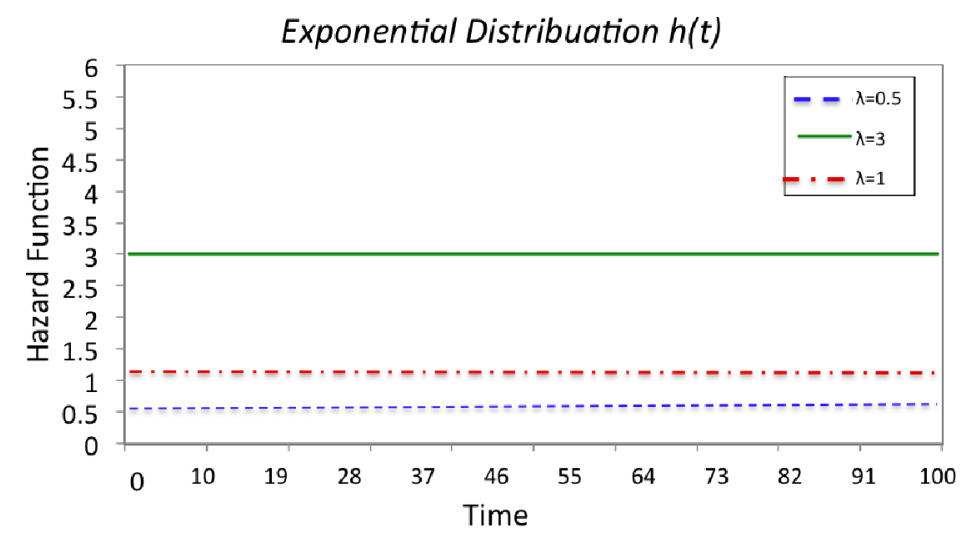

Figure 2.5: Hazard function for exponential distribution with $\lambda=0.5,1,3$.

\section{Exponential Disribution $f(t)$}

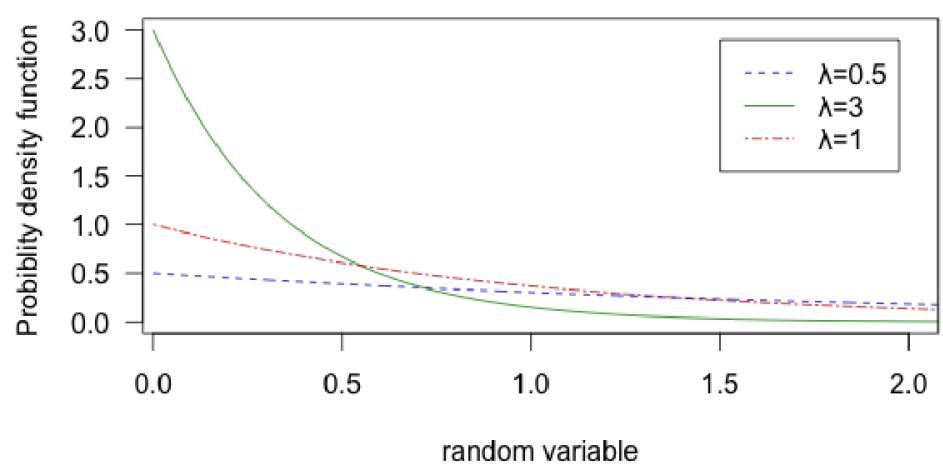

Figure 2.6: Probability density function for exponential distribution with $\lambda=0.5,1,3$.

\subsubsection{Weibull distribution}

The Weibull model $(T \sim W(\lambda, \gamma))$ is the most frequently applied parametric survival model. It was introduced by Waloddi Weibull (1939) and is a generalization of the exponential model with two positive parameters $\lambda$ called a scale parameter, and $\gamma$ known as a shape parameter. The latter parameter, $\gamma$, allows great flexibility to the model since the Weibull hazard function can take a variety of forms depending on the value of this shape parameter $\gamma$. In the particular case of $\gamma=1$, the hazard function takes a constant value $\lambda$, so the survival times 
have exponential distribution as a special case of Weibull distribution. For other values of $\gamma$, the hazard function increases or decreases monotonously for $\gamma>1$ and $\gamma<1$, respectively. The form of Weibull hazard and density functions for different values of $\gamma$ and $\lambda$ are shown in Figure 2.7. An overview of Weibull distribution is given in Murthy, Xie and Jiang [22].
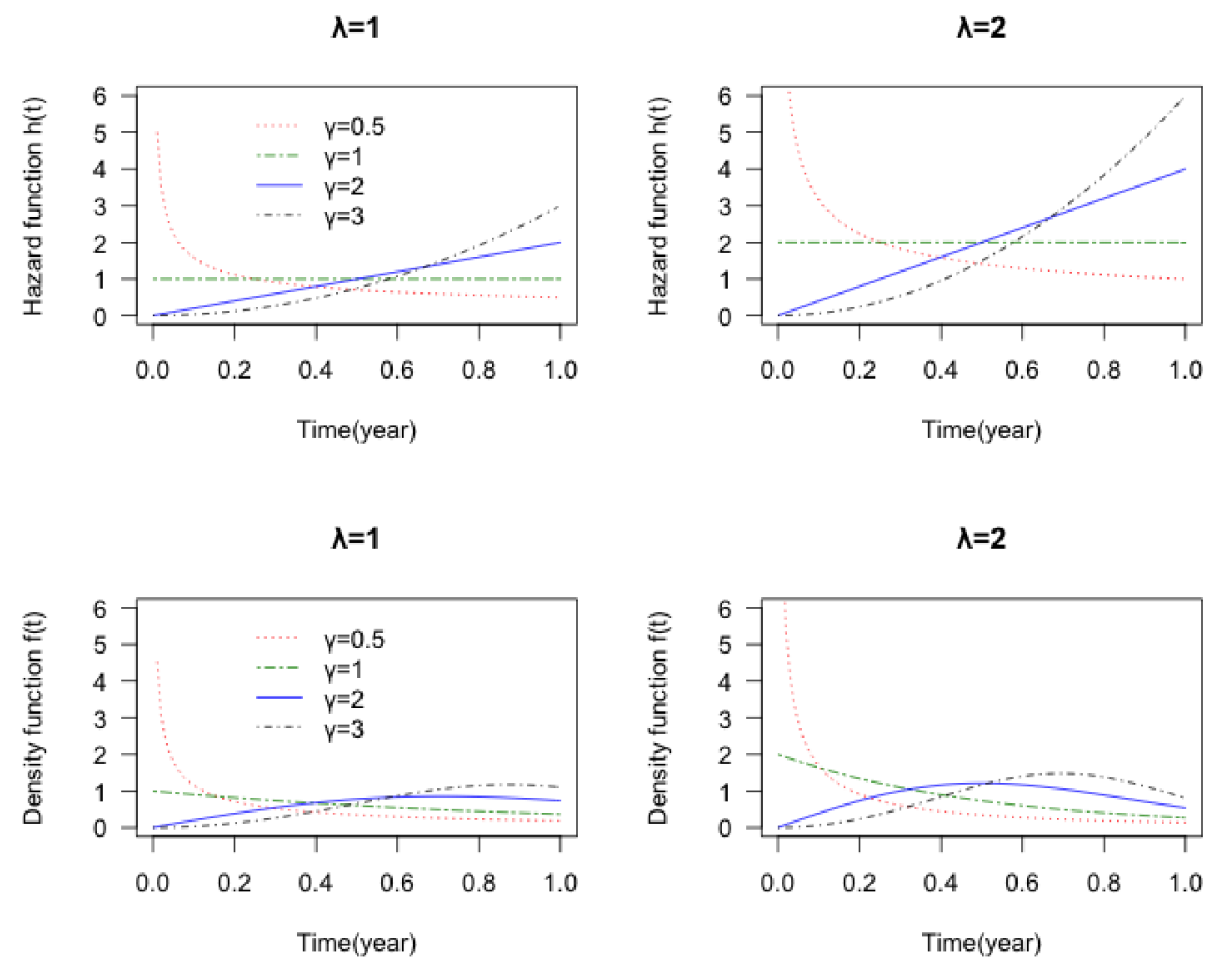

Figure 2.7: Hazard and density functions for Weibull survival times with different values of shape parameter $\gamma=(0.5,1,2,3)$ and scale parameter $\lambda=(1,2)$.

For the Weibull distribution, the general form of the hazard function and the corresponding probability density function, survival function, cumulative hazard function, mean, and variance can be derived easily as

$$
\begin{array}{r}
\text { probability density function, } f(t)=\lambda \gamma t^{\gamma-1} e^{-\lambda t^{\gamma}},(\gamma>0, \lambda>0) \\
\text { survival function, } S(t)=e^{-\lambda t^{\gamma}} \\
\text { hazard function, } h(t)=\lambda \gamma t^{\gamma-1} \\
\text { cumulative hazard function, } \quad H(t)=\lambda t^{\gamma}
\end{array}
$$




$$
\begin{gathered}
\text { mean, } E(T)=\lambda^{-1 / \gamma} \Gamma(1+1 / \gamma) \\
\text { variance, } \quad V(T)=\lambda^{-2 / \gamma}\left(\Gamma\left(1+2 / \gamma-\Gamma(1+1 / \gamma)^{2}\right),\right.
\end{gathered}
$$

where the exception and variance are not of simple form as they are based on a gamma function $\Gamma$ defined by $\Gamma(v)=\int_{0}^{\infty} s^{v-1} e^{-v} d v \quad(v>0)$.

Therefore, the Weibull regression model, which is also a proportional hazards (PH) model, is defined by one of the following functions in which we assume that the linear function $\boldsymbol{x}^{\prime} \boldsymbol{\beta}$ does not include an intercept term:

$$
\begin{aligned}
h(t \mid \boldsymbol{x}) & =\lambda \gamma t^{\gamma-1} \exp \left(\boldsymbol{x}^{\prime} \boldsymbol{\beta}\right), \\
S(t \mid \boldsymbol{x}) & =\exp \left[-\lambda t^{\gamma} \exp \left(\boldsymbol{x}^{\prime} \boldsymbol{\beta}\right),\right. \\
H(t \mid \boldsymbol{x}) & =\lambda t^{\gamma} \exp \left(\boldsymbol{x}^{\prime} \boldsymbol{\beta}\right) .
\end{aligned}
$$

\subsection{Fitting Parametric Survival Models}

Parametric models can be fitted to observed data of survival times to estimate the unknown parameters of the survival function, $S(t)$, by using the maximum likelihood (ML) method. Let us consider survival data where one or more censored survival times are observed for $N$ individuals. Let $T$ be a random variable denoting time until the event occurs, and let $T_{i}$ be the survival time for the $i^{\text {th }}$ individual, and $Y_{i}$ be the minimum of the censoring time $C_{i}$ and the event time $T_{i}$, defined by $Y_{i}=\min \left(T_{i}, C_{i}\right)$. If the survival time $Y_{i}$ is uncensored, then the observed failure time contributes to the likelihood as the probability density function $f\left(Y_{i}\right)$. However, if $Y_{i}$ is a censored time (i.e., $Y_{i}=C_{i}$ ), we know that the failure time of an individual $T_{i}$ is greater than $Y_{i}$ (i.e., $T_{i}>C_{i}$ ), so that the probability of the event is equal to $P\left(T_{i}>Y_{i}\right)$. This probability is $S\left(Y_{i}\right)$ and contributes to the likelihood function, and we cannot ignore the censored observations related to the survival experience for those individuals. 
Therefore, the joint likelihood function over all observations $i=1, \ldots, N$ is

$$
L=\prod_{i: Y_{i} \text { uncensored }}^{N} f\left(Y_{i}\right) \prod_{i: Y_{i} \text { censored }}^{N} S\left(Y_{i}\right) .
$$

Suppose that the observed data are $N$ pairs of observations so that the pair of the $i^{\text {th }}$ individual is $\left(t_{i}, \delta_{i}\right), i=1, \ldots, N$, where $\delta_{i}$ is a censored indicator $\left(\delta_{i}=0\right.$ if $T_{i}$ is rightcensored; $\delta_{i}=1$ otherwise). Then the likelihood can be alternatively expressed as

$$
L=\prod_{i=1}^{N}\left\{f\left(t_{i}\right)\right\}^{\delta_{i}}\left\{S\left(t_{i}\right)\right\}^{1-\delta_{i}} .
$$

As this likelihood is a function of the unknown parameters in the probability density and survival functions, it can be maximized with respect to these unknown parameters. From the relationships among survival, hazard, and probability density functions presented in section (2.2), the likelihood function can be rewritten as

$$
L=\prod_{i=1}^{N}\left\{\frac{h\left(t_{i}\right.}{S\left(t_{i}\right)}\right\}^{\delta_{i}} S\left(t_{i}\right)
$$

or, equivalently,

$$
L=\prod_{i=1}^{N}\left\{h\left(t_{i}\right)\right\}^{\delta_{i}} S\left(t_{i}\right) .
$$

In practice, working with the logarithm of the likelihood function is a more convenient method to estimate the unknown parameters.

The log-likelihood can be written as

$$
\log L=\sum_{i=1}^{N} \delta_{i} \log h\left(t_{i}\right)+\sum_{i=1}^{N} \log S\left(t_{i}\right)
$$

which is equivalent to 


$$
\log L=\sum_{i=1}^{N} \delta_{i} \log h\left(t_{i}\right)-\sum_{i=1}^{N} H\left(t_{i}\right)
$$

\subsubsection{Fitting the exponential distribution}

Suppose that the survival times of $N$ individuals $t_{1}, t_{2}, \ldots, t_{N}$ have an exponential distribution with the mean $1 / \lambda$.

Recalling (2.4.1) for exponential distribution, we have

$$
h(t)=\lambda ; S(t)=\exp (\lambda t)
$$

In this case, the likelihood function (1.14) becomes

$$
L(\lambda)=\prod_{i=1}^{N} \lambda^{\delta_{i}} \exp \left(-\lambda t_{i}\right)
$$

and the log-likelihood function takes the form

$$
\log L(\lambda)=\sum_{i=1}^{N} \delta_{i} \log \lambda-\lambda \sum_{i=1}^{N} t_{i}
$$

Let $\sum_{i=1}^{N} \delta_{i}=r$ denote the number of uncensored survival times (observed times) in the data.

Then the log-likelihood function becomes

$$
\log L(\lambda)=r \log \lambda-\lambda \sum_{i=1}^{N} t_{i}
$$

To estimate the unknown parameter $\lambda$, we need to differentiate the log-likelihood function with respect to $\lambda$ and equate the derivative to zero (as described in Collett [9]).

We have

$$
\frac{\partial \log L(\lambda)}{\partial \lambda}=\frac{r}{\lambda}-\sum_{i=1}^{N} t_{i}
$$


and

$$
\frac{\partial^{2} \log L(\lambda)}{\partial \lambda^{2}}=\frac{-r}{\lambda^{2}} .
$$

So the maximum likelihood estimator of $\lambda$ is

$$
\hat{\lambda}=\frac{r}{\sum_{i=1}^{N} t_{i}} .
$$

For the exponential proportional hazards model, $T_{i}$ is exponentially distributed denoted as $T_{i} \sim \operatorname{Exp}\left(\lambda \exp \left(\boldsymbol{x}_{i}^{\prime} \boldsymbol{\beta}\right)\right)$.

Therefore, the likelihood function of this parametric survival model is given by

$$
\begin{aligned}
L(\lambda ; \boldsymbol{\beta} \mid \boldsymbol{x}) & =\prod_{i=1}^{N}\left\{h\left(t_{i}\right)\right\}^{\delta_{i}} S\left(t_{i}\right) \\
& =\prod_{i=1}^{N}\left\{h_{0}(t) \exp \left(\boldsymbol{x}_{i}^{\prime} \boldsymbol{\beta}\right)\right\}^{\delta_{i}} e^{-\int_{0}^{t_{i}} h(t ; \boldsymbol{\beta}) d t}, \quad\left(h_{0}(t)=\lambda\right) \\
& =\prod_{i=1}^{N}\left\{\lambda \exp \left(\boldsymbol{x}_{i}^{\prime} \boldsymbol{\beta}\right)\right\}^{\delta_{i}} e^{-\lambda t_{i} \exp \left(\boldsymbol{x}_{i}^{\prime} \boldsymbol{\beta}\right)}, \quad\left(\lambda=e^{\beta_{0}}\right) .
\end{aligned}
$$

Then the likelihood function for the regression parameters becomes

$$
L(\boldsymbol{\beta} \mid \boldsymbol{x})=\prod_{i=1}^{N}\left\{\exp \left(\boldsymbol{x}_{i}^{\prime} \boldsymbol{\beta}\right)\right\}^{\delta_{i}} e^{-\exp \left(\boldsymbol{x}_{i}^{\prime} \boldsymbol{\beta}\right) t_{i}},
$$

and the corresponding log-likelihood can be written as

$$
\log L(\boldsymbol{\beta} \mid \boldsymbol{x})=\sum_{i=1}^{N} \delta_{i}\left(\exp \left(\boldsymbol{x}_{i}^{\prime} \boldsymbol{\beta}\right)-\sum_{i=1}^{N} \exp \left(\boldsymbol{x}_{i}^{\prime} \boldsymbol{\beta}\right) t_{i}\right.
$$

From this log-likelihood function, the first and second derivatives with respect to $\boldsymbol{\beta}$ are obtained as 


$$
\begin{gathered}
\left.\frac{\partial \log L(\boldsymbol{\beta} \mid \boldsymbol{x})}{\partial \boldsymbol{\beta}}\right|_{\boldsymbol{\beta}=0}=-\sum_{i=1}^{N} \exp \left(\boldsymbol{x}_{i}^{\prime} \boldsymbol{\beta}\right) \boldsymbol{x}_{i} t_{i}+\sum_{i=1}^{N} \delta_{i} \boldsymbol{x}_{i} \\
\left.\frac{\partial^{2} \log L(\boldsymbol{\beta} \mid \boldsymbol{x})}{\partial \boldsymbol{\beta} \partial \boldsymbol{\beta}^{\prime}}\right|_{\beta=0}=-\sum_{i=1}^{N} \exp \left(\boldsymbol{x}_{i}^{\prime} \boldsymbol{\beta}\right) \boldsymbol{x}_{i} \boldsymbol{x}_{i}^{\prime} t_{i}
\end{gathered}
$$

Then the parameter estimates $\hat{\boldsymbol{\beta}}$ can be obtained by using the iterative Newton-Raphson method. The iterative equations take the form

$$
\boldsymbol{\beta}^{(m+1)}=\boldsymbol{\beta}^{(m)}+\left(-\frac{\partial^{2} \log L(\boldsymbol{\beta})}{\partial \boldsymbol{\beta} \partial \boldsymbol{\beta}^{\prime}}\right)_{\boldsymbol{\beta}^{(m)}}^{-1}\left(\frac{\partial \log L(\boldsymbol{\beta})}{\partial \boldsymbol{\beta}}\right)_{\boldsymbol{\beta}^{(m)}},
$$

for $m=0,1,2, \ldots$, starting with some initial value $\boldsymbol{\beta}^{(0)}$ of $\boldsymbol{\beta}$.

\subsubsection{Fitting the Weibull distribution}

In this situation, the survival times of $N$ individuals are assumed to be a censored sample from a Weibull distribution with the shape parameter $\gamma$ and scale parameter $\lambda$. From the probability density, hazard, and survivor function of Weibull distribution $W(\lambda, \gamma)$, which is presented in section (2.4.2), the likelihood function of $N$ survival times is

$$
L(\lambda, \gamma)=\prod_{i=1}^{N}\left\{\lambda \gamma t_{i}^{\gamma-1}\right\}^{\delta_{i}} \exp \left(-\lambda t_{i}^{\gamma}\right)
$$

and the corresponding log-likelihood function is

$$
\log L(\lambda, \gamma)=\sum_{i=1}^{N} \delta_{i} \log (\lambda \gamma)+(\gamma-1) \sum_{i=1}^{N} \delta_{i} \log t_{i}-\lambda \sum_{i=1}^{N} t_{i}^{\gamma}
$$

Let $\sum_{i=1}^{N} \delta_{i}=r$ denote the number of uncensored survival times. Then the log-likelihood can be written as 


$$
\log L(\lambda, \gamma)=r \log (\lambda \gamma)+(\gamma-1) \sum_{i=1}^{N} \delta_{i} \log t_{i}-\lambda \sum_{i=1}^{N} t_{i}^{\gamma}
$$

The ML estimator of $\lambda, \gamma$ can be found by taking the derivative of this log-likelihood with respect to $\lambda, \gamma$, and equating this derivative to zero. The estimating equations for these two parameters are

$$
\begin{gathered}
\frac{\partial \log L(\lambda, \gamma)}{\partial \lambda}=\frac{r}{\lambda}-\sum_{i=1}^{N} t_{i}^{\gamma}=0 \\
\frac{\partial \log L(\lambda, \gamma)}{\partial \gamma}=\frac{r}{\gamma}+\sum_{i=1}^{N} \delta_{i} \log t_{i}-\hat{\lambda} \sum_{i=1}^{N} t_{i}^{\gamma} \log t_{i}=0 .
\end{gathered}
$$

The maximum likelihood estimator of $\lambda$ is obtained as

$$
\hat{\lambda}=\frac{r}{\sum_{i=1}^{N} t_{i}^{\hat{\gamma}}}
$$

which depends on the estimate $\hat{\gamma}$, which may be obtained by solving the equation

$$
\frac{r}{\gamma}+\sum_{i=1}^{N} \delta_{i} \log t_{i}-\frac{r}{\sum_{i=1}^{N} t_{i} \hat{\gamma}} \sum_{i=1}^{N} t_{i}^{\gamma} \log t_{i}=0
$$

Since this equation is non-linear, it can be solved by using an iterative numerical procedure such as the Newton-Raphson method.

For the Weibull regression model, the event time $T_{i}$ follows a Weibull distribution, denoted by $T_{i} \sim W\left(\lambda \exp \left(\boldsymbol{x}_{i}^{\prime} \boldsymbol{\beta}\right), \gamma\right)$, so that all subjects are Weibull with the same shape parameter but vary with respect to the scale parameter.

The likelihood of parameters is defined as

$$
L(\lambda ; \gamma ; \boldsymbol{\beta} \mid \boldsymbol{x})=\sum_{i=1}^{N}\left\{\lambda \gamma t_{i}^{\gamma-1} \exp \left(\boldsymbol{x}_{i}^{\prime} \boldsymbol{\beta}\right)\right\}^{\delta_{i}} \exp \left(-\lambda t_{i}^{\gamma} \exp \left(\boldsymbol{x}_{i}^{\prime} \boldsymbol{\beta}\right)\right)
$$


The log-likelihood is

$\log L(\lambda ; \gamma ; \boldsymbol{\beta} \mid \boldsymbol{x})=\sum_{i=1}^{N} \delta_{i} \log (\lambda \gamma) \exp \left(\boldsymbol{x}_{i}^{\prime} \boldsymbol{\beta}\right)+(\gamma-1) \sum_{i=1}^{N} \delta_{i} \log t_{i} \exp \left(\boldsymbol{x}_{i}^{\prime} \boldsymbol{\beta}\right)-\lambda \sum_{i=1}^{N} \exp \left(\boldsymbol{x}_{i}^{\prime} \boldsymbol{\beta}\right) t_{i}^{\gamma}$

Subsequently, an iterative method such as the Newton-Raphson method is used to compute the maximum likelihood estimators (MLEs) of all parameters $\lambda, \gamma$ and $\boldsymbol{\beta}$. Once these estimates are obtained, the estimated variance-covariance matrix and other estimated quantities such as hazard, cumulative hazard, and survivor functions can be obtained as

$$
\begin{gathered}
\hat{h}(t \mid \boldsymbol{x})=\hat{\lambda} \hat{\gamma} t^{\hat{\gamma}-1} \exp \left(\boldsymbol{x}^{\prime} \hat{\boldsymbol{\beta}}\right), \\
\hat{H}(t \mid \boldsymbol{x})=\hat{\lambda} t^{\hat{\gamma}} \exp \left(\boldsymbol{x}^{\prime} \hat{\boldsymbol{\beta}}\right), \\
\hat{S}(t \mid \boldsymbol{x})=\exp [-\hat{H}(t \mid \boldsymbol{x})] .
\end{gathered}
$$

Weibull distributions are often used to model survival times because they are able to describe the actual evolution of the hazard function in an appropriate way in many situations (an illustrative example is given in Collett [9]). 


\section{Chapter 3}

\section{Frailty Models for Recurrent Event Data}

\subsection{Overview of Recurrent Event Data}

In previous sections, we describe briefly the ordinary univariate data where for each subject a single time is recorded and all subjects are independent. But one might consider studying repeated cases or measurements of the same event for a given subject over the follow-up times. Such processes arise frequently in survival analysis, especially in clinical trials, and are referred to as "recurrent event processes" and the data they provide are called "recurrent event data". In clinical trails, examples include the occurrence of multiple episodes of relapses from remission, recurrent heart attacks of coronary patients as well as repeated asthma attacks of asthma patients, and recurrence of bladder cancer tumours in a cohort of patients randomized to different treatment groups. In demographic studies, examples include repeated events such as pregnancy, divorce, marriage, or leaving the house. In business, examples include the filing of insurance claims by policy holders or warranty claims on vehicles. In criminal aspects, police investigators might be interested in studying recurrent events of arrests such as sexual assault, theft, or homicide. There are several objectives in 
analyzing recurrent event data; one is to asses the relationship of fixed covariates, treatments, and time-varying factors with the rate of event occurrence. In addition, such data analysis helps researchers to describe individual event processes as well as to compare groups of processes. An important consideration must be taken in the analysis of recurrent event data. The choice of an analysis technique depends on whether all recurrent events on the same subject are treated as identical or different types of events. According to Kleinbaum and Klein [20], the "Counting Process Approach" is used to analyze recurrent events that are considered to be identical, while an alternative approach that involve "Stratified Cox (SC) Models" can be used to analyze those repeated events that are different. See Cook and Lawless [11] for a comprehensive review and discussion on recurrent events.

\subsection{Frailty Models}

It is important to note that a random effects model takes into account the effects of unobservable heterogeneity that may be caused by different sources. The concept of frailty is to introduce random effects in the survival model to account for association and unobserved heterogeneity. In its simplest form, a frailty is an unobserved random factor that changes multiplicatively the hazard function of an individual or a group or cluster of individuals (see, for example, Duchateau and Janssen [13], Wienke [31], Therneau and Grambsch [27], and Hougaard [17]). In fact, the term frailty is introduced by Vaupel et al.(1979) when they used it in univariate survival models, and later the model is promoted by Clayton (1978) for applications in multivariate situations. This frailty, denoted by $u$, is a term that describes the common risk or the individual heterogeneity, acting as a factor on the hazard function. Two categories of frailty models can be pointed out. The first one is the univariate frailty models that take into account univariate survival times, and the second one is the multivariate frailty models that consider multivariate survival times. Frailty models can deal with more than one frailty term (Duchateau and Janssen [13]), but in this thesis we will discuss 
the case of one frailty term within the same cluster or subject.

The frailty is also defined as a mixture variable used to describe unknown source of different risks in population, and it is assumed to follow some distribution (Wienke [31]). Throughout this thesis, we will assume the frailties $u_{i}$ 's are independent and follow a normal distribution with mean $E\left(u_{i}\right)=0$ and variance $\operatorname{var}\left(u_{i}\right)=\sigma_{u}^{2}$. This parameter $\sigma_{u}^{2}$ will provide information related to the variability (heterogeneity) across the population of clusters.

\subsubsection{Univariate Frailty Models}

We first consider a simple data set with one level of clustering known as the "univariate data". Such data consist of a single cluster and all time variables for the same type of event and individuals are assumed to be independent (Hougaard [18]). Basic survival models are applied for independent and identically distributed data analysis. In this case, a basic model assumes that the study population is homogeneous with respect to some observed predictor variables. To be more specific, those models deal with the survival data of different individuals that are assumed to be independent from others so that the individual survival times are also independent and identically distributed (i.e., failure times follow the same distribution). However, according to Wienke [31], individuals vary significantly in most circumstances due to the impact of a certain treatment or the great difference with respect to the influence of various explanatory variables (risk factors). This variability is often refereed to as heterogeneity and it is generally considered to be one of the most important source of variability in several statistical studies such as biological, medical, and epidemiological ones. The models fitted to such data are called "univariate frailty models", which was introduced in survival analysis by Vaupel et al. (1979) to address the problem of unobserved heterogeneity in the study population. The main concept of these models is that all individuals have different frailties, which lead to a change into the individual hazard so that all individuals sampled into that study are subject in principle under different level of risk (e.g., risk of death, risk of disease recurrence). In this sense, the more frail individuals tend to have 
the event earlier than those who are less frail. A frailty model in the univariate case acts multiplicatively on the baseline hazard function, so the conditional hazard function on frailty is in the form

$$
h(t \mid u)=u h_{0}(t)
$$

where $u$ is the frailty term considered to be a positive random variable that is assumed to follow some distribution and differs across the population; $h_{0}(t)$ is the baseline hazard function that can be modelled non-parametrically or parametrically(e.g., Weibull, exponential, gamma, log-normal, Gompertz). Those individuals with $u>1$ are more frail caused by unexplained covariates and will have higher risk of failure, whereas those with $u<1$ are less frail and will tend to survive longer.

Note that observed explanatory variables could also be included in the analysis by using the proportional hazards model that is conditional on the random effect (frailty) (Vaida and Ronghui [28]). In this case, the conditional hazard function on frailty and covariates for the $i^{\text {th }}$ individual can be written in the form

$$
h(t \mid \boldsymbol{x}, u)=u h_{0}(t) \exp \left(\boldsymbol{x}^{\prime} \boldsymbol{\beta}\right)
$$

where $\boldsymbol{x}=\left(x_{1}, \ldots, x_{p}\right)^{\prime}$ and $\boldsymbol{\beta}=\left(\beta_{1}, \ldots, \beta_{p}\right)$ are vectors of covariates and regression coefficients, respectively.

As a result, the frailty model is considered to be a generalization of the proportional hazards (PH) model, which is the best known as the Cox model (Cox [12]). The ordinary proportional hazards $(\mathrm{PH})$ model (2.10) can be obtained if the variation of frailty variable $u$ is equal to zero for all individuals. Conditional on the unobserved frailty term $\left(u_{i}\right)$, the likelihood function of the data $\left(t_{i}, \delta_{i}, \boldsymbol{x}_{\boldsymbol{i}}, u_{i}\right)$ is given by

$$
L=\prod_{i=1}^{N}\left\{u_{i} h_{0}(t) \exp \left(\boldsymbol{x}_{\boldsymbol{i}}{ }^{\prime} \boldsymbol{\beta}\right)\right\}^{\delta_{i}} \exp \left\{-u_{i} H_{0}(t) \exp \left(\boldsymbol{x}_{\boldsymbol{i}}{ }^{\prime} \boldsymbol{\beta}\right)\right\}, \text { for } i=1, \ldots, N
$$


According to Wienke [31], the random effect can be integrated out by numerical integration or Monte Carlo approximation based on the distribution of frailty in order to obtain unconditional likelihood function (not depending on unobserved quantities). The survival function of an individual conditional on the frailty $u$ without observed covariates can be expressed as

$$
S(t \mid u)=e^{-\int_{0}^{t} h(s \mid u) d s}=e^{-u \int_{0}^{t} h_{0}(s) d s}=e^{u H_{0}(t)} .
$$

Up to now, the model is described at the individual level (conditional), and data for this model are not observed. The marginal survival function can be obtained from the conditional survival function, $S(t \mid u)$, when we integrate out the frailty term. The marginal survival function is the expectation of the conditional survival function with weights given by the density function of the frailty distribution. That is,

$$
S(t)=E[S(t \mid u)]=E\left[e^{-u H_{0}(t)}\right] .
$$

\subsubsection{Multivariate Frailty Models}

Another significant application of frailty models in survival analysis is dealing with cluster or repeated data, referred to as "multivariate survival data". For instance, lifetimes of relatives or recurrent events for the same person such as infections are considered to be clustered survival times. In such data, the assumption of independent lifetimes (i.e., times-to-event, failure times, duration times) for clusters might not hold in many cases. Therefore, the frailty models for multivariate survival data were introduced by Clayton (1978), which can be used to model correlated survival times within clusters (clusters could be individuals, subjects, or groups). The frailty model in survival analysis is widely used, as it allows us to use heterogeneity between clusters, and to make inference on frailty term and the heterogeneity parameter (Hougaard [18] and Wienke [31]). This model is considered to be a mixed model due to the assumption of common frailty (the risk) for all individuals within a cluster, and 
it is used to measure the association between multiple outcomes in clusters. According to Wienke [31], frailty models for multivariate survival data are derived under conditional independence assumption by specifying unobserved variables that act multiplicatively on the baseline hazard. In case of covariates in survival analysis, the frailty model is an extension of the proportional hazards model consisting of three components: baseline hazard function, exponential term of explanatory variables, and a random effect for each individual so that the hazard function of an individual becomes conditional on an unobserved random effect $u_{i}$. In the following sections, the important concepts of both shared and correlated frailty models are outlined and compared briefly.

\subsubsection{Shared Frailty Models for Recurrent Events}

A shared frailty model is a random effects model where individuals within a cluster share the same value of frailty and those frailties are randomly distributed across clusters (Hougaard [18]). It is a conditional independence model in which all event times in a cluster are assumed to be independent given the frailty variables. The frailty value is constant over time and common to the individuals in a group so that sharing the frailty value generates dependence between those individuals within the group (cluster). In survival analysis, the shared frailty model is considered as a mixture model (a random effects model) with two sources of variation: the group variation described by the random variable $u$ (the frailty) and individual variation described by the hazard function (Wienke [31]). Conditional on frailty

$u_{i}$, the joint conditional multivariate survival function for individuals in the $i^{\text {th }}$ cluster can be derived as

$$
\begin{aligned}
S\left(t_{i 1}, \ldots, t_{i n_{i}} \mid \boldsymbol{x}_{i}, u_{i}\right) & =S\left(t_{i 1} \mid \boldsymbol{x}_{i 1}, u_{i}\right) \ldots S\left(t_{i n_{i}} \mid \boldsymbol{x}_{i n_{i}}, u_{i}\right) \\
& =\exp \left(-u_{i} \sum_{j=1}^{n_{i}} H_{0}\left(t_{i j}\right) e^{\left(\boldsymbol{x}_{i j}^{\prime} \boldsymbol{\beta}\right)}\right)
\end{aligned}
$$


where $H_{0}(t)=\int_{0}^{t} h_{0}(s) d s$ is the cumulative baseline hazard function; $\boldsymbol{x}_{i}=\left(\boldsymbol{x}_{i 1}, \ldots, \boldsymbol{x}_{i n i}\right)$ is the covariates matrix of individuals in the $i^{\text {th }}$ cluster.

In case the frailty variation is zero, the event times in a cluster are independent; otherwise those event times are positively dependent (Wienke [31]). However, it is assumed that there is independence between event times from different clusters. The main concept of a shared frailty model is that all individuals $j\left(j=1, \ldots, n_{i}\right)$ in a cluster $i$ share the same value of frailty $u_{i}(i=1, \ldots, N)$ and hence the term shared frailty model. More details about shared frailty models can be found in Duchateau and Janssen [13], Hougaard [18], and Wienke [31].

\subsubsection{The Parametric Proportional Hazards Models (PH) with Shared Frailty}

For subject $j\left(j=1, \ldots, n_{i}\right)$, from cluster $i(i=1, \ldots, N)$, we observe $y_{i j}$, the minimum of the censoring time $c_{i j}$ and the event time $t_{i j}$, denoted as $y_{i j}=\min \left(t_{i j}, c_{i j}\right)$, and $\delta_{i j}$ is the censoring indicator $\left(\delta_{i j}=I\left(t_{i j} \leq c_{i j}\right)\right)$. In this thesis, we assume that $t_{i j}$ 's and $c_{i j}$ 's are independent. Suppose the number of observed events in the $i^{\text {th }}$ cluster is $d_{i}=\sum_{j=1}^{n_{i}} \delta_{i j}$.

The shared frailty model can be defined as

$$
h_{i j}\left(t \mid u_{i}\right)=h_{0}(t) \exp \left(\boldsymbol{x}_{i j}^{\prime} \boldsymbol{\beta}+u_{i}\right)
$$

where $h_{i j}(t)$ is the conditional hazard function on the frailty $u_{i}$ for the $j^{t h}$ subject from the $i^{\text {th }}$ cluster; $\boldsymbol{\beta}$ is a vector of unknown regression coefficients and $h_{0}(t)$ is the baseline hazard function; $\boldsymbol{x}_{i j}$ is a vector of covariates and $u_{i}$ is the random effect (the frailty) for the $i^{t h}$ cluster with a density function $f\left(u_{i}\right)$. In the case of Weibull $\mathrm{PH}$ regression, the conditional hazard for an individual is given by

$$
h_{i j}\left(t \mid u_{i}\right)=h_{0}(t) \exp \left(\boldsymbol{x}_{i j}^{\prime} \boldsymbol{\beta}+u_{i}\right) \gamma t^{\gamma-1}
$$

and the conditional survival function is 


$$
S_{i j}\left(t \mid u_{i}\right)=\exp \left\{-h_{0}(t) \exp \left(\boldsymbol{x}_{i j}^{\prime} \boldsymbol{\beta}+u_{i}\right) t^{\gamma}\right\}
$$

Throughout this thesis, an exponential baseline hazard (special case of Weibull distribution) is assumed and the frailties $u_{i}$ 's are assumed to be independent and follow a normal distribution. Similar to the univariate case, the likelihood for the parametric shared normal frailty model with $N$ clusters of size $n_{i}(i=1, \ldots, n)$ is given by

$L\left(\boldsymbol{\theta}, \boldsymbol{\beta}, \sigma_{u}^{2}\right)=\prod_{i=1}^{N} \int_{0}^{\infty} \prod_{i=1}^{n_{i}}\left\{h_{0}\left(t_{i j} ; \boldsymbol{\theta}\right) \exp \left(\boldsymbol{x}_{i j}^{\prime} \boldsymbol{\beta}+u_{i}\right)\right\}^{\delta_{i}} \exp \left\{-H_{0}\left(t_{i j} ; \boldsymbol{\theta}\right) \exp \left(\boldsymbol{x}_{i j}^{\prime} \boldsymbol{\beta}+u_{i}\right)\right\} f\left(u_{i} ; \sigma_{u}^{2}\right) d u_{i}$

with

$$
f\left(u_{i} ; \sigma_{u}^{2}\right)=\frac{1}{\sigma_{u} \sqrt{2 \pi}} e^{-\frac{u_{i}^{2}}{2 \sigma_{u}^{2}}}
$$

denoting the density function of a normal distribution with mean $E\left(u_{i}\right)=0$ and variance $\operatorname{var}\left(u_{i}\right)=\sigma_{u}^{2}$. The regression parameters $\boldsymbol{\beta}$, parameter vector $\boldsymbol{\theta}$ of baseline hazard function $h_{0}(t)$, and variance of the frailty $\sigma_{u}^{2}$ can be estimated by numerically maximizing the likelihood function (3.5).

\subsubsection{Correlated Frailty Models for Recurrent Events}

As discussed in the previous section, the shared frailty model is beneficial for clustered or repeated events time data to model the correlation between event times in a cluster. It is used in the case of multivariate events time data, and is considered as a special case of the correlated frailty model with correlations between the frailties equal to one. Similar to univariate and shared frailty models, the correlated frailty model is a mixed model because the frailty for each individual is assumed to be random. In a correlated frailty model, event times in a cluster are assumed independent given a vector of frailty, where the frailty variables are correlated but not necessarily shared by all individuals within a cluster. In other words, 
related individuals in a cluster have different but dependent frailties (some discussion about the correlated frailty model can be found in Vaida and Ronghui [28] and Wienke [31]). 


\section{Chapter 4}

\section{Score Test}

\subsection{Introduction}

As previously mentioned, frailty models are used to model survival data when different individuals share some common feature or to study the common factor among recurrent event times for the same individual. Frailty is used to measure the individual heterogeneity that is unexplained by the observed risk variables included in the model. In many applications of survival data, frailty models are used to describe this heterogeneity (see Blossfeld and Hamerle [4], Box-Steffensmeier, De Boef, and Joyce [5], McGilchrist and Aisbett [21], Aalen [2], and Hougaard [17]).

In this thesis, we are interested in testing the homogeneity of individuals in recurrent events data or groups in clustered survival data for given explanatory variables (i.e. testing the independence of individuals within groups or repeated measures within individuals). The score test for testing variance components in frailty models is convenient to apply as compared to the likelihood ration test, as it only requires an estimation of the model parameters under the null hypothesis of no frailty. This score statistics for one-sided tests asymptotically follows a mixture of chi-squared distributions. (Silvapulle and Silvapulle [24] , Fitzmaurice, Lipsitz, and Ibrahim [14] and Sinha [25],[26]). 
In the following section, we describe the score test for testing whether the variance component of a frailty is equal to zero ,or equivalently, whether the individuals or groups are homogeneous for given risk variables.

\subsection{The Score Test}

We apply the idea of one-sided score test for testing homogeneity between individuals in clustered data as proposed by Silvapulle and Silvapulle [24] and implemented later by Claeskens, Nguti and Janssen [7], and Sinha [26]. This type of hypothesis testing problem is called a one-sided testing since the heterogeneity parameter must be positive and we are to test $H_{0}: \sigma_{u}^{2}=0$ against $H_{a}: \sigma_{u}^{2}>0$ (see Claeskens, Nguti, and Janssen [7]). According to Sinha [26], Fitzmaurice, Lipsitz, and Ibrahim [14], Commenges and Andersen [10], Claeskens, Crainiceanu and Ruppert [8], Nguti and Janssen [7], and Verbeke and Molenberghs [29], the heterogeneity parameter $\sigma_{u}^{2}$ is on the boundary of parameter space $[0, \infty)$ under the null hypothesis $H_{0}: \sigma_{u}^{2}=0$, which leads the score statistics $T$ for the one-sided alternative hypothesis $H_{a}: \sigma_{u}^{2}>0$ to follow a mixture of chi-squared distributions, denoted as $0.5 \chi_{0}^{2}+0.5 \chi_{1}^{2}$, when the number of individuals tend to infinite $(N \longrightarrow \infty)$. Following Sinha [26], we obtain an appropriate score statistic to test the homogeneity of individuals among clusters using the proportional hazards model with a shared frailty. We consider a set of $N$ individuals, clusters or groups. For each cluster $i(i=1, \ldots \ldots, N)$ we observe a vector of $n_{i}$ repeated event times denote as $T_{i j}$ with a censoring indicator $\delta_{i j}$ which takes the value 1 if the event of interest $T_{i j}$ is observed or 0 if $T_{i j}$ is right-censored. In this thesis, we assume the censoring time $C_{i j}$ is right-censored, non-informative, and independent from the true event times.

\subsubsection{The Model Structure}

Following Sinha [26], we use a shared frailty model in which the dependence of survival times within a cluster is described by the independent frailty variables $u_{1}, \ldots, u_{N}$. We consider a 
proportional hazards model for the $j^{\text {th }}$ event time of the $i^{\text {th }}$ individual conditional on random effects $u_{i}$, given by

$$
h_{i j}(t)=h_{0}(t) \exp \left(\boldsymbol{x}_{i j}^{\prime} \boldsymbol{\beta}+u_{i}\right)
$$

where $h_{0}(t)=h_{0}(t, \boldsymbol{\theta})$ is a baseline hazard function depending on $\theta$, and $u_{i}$ are the frailties that are assumed to be independent and normally distributed with mean 0 and unknown variance component $\sigma_{u}^{2}$.

For given data $\left(T_{i 1}, \delta_{i 1}, x_{i 1}\right), \ldots . .,\left(T_{i n_{i}}, \delta_{i n_{i}}, x_{i n_{i}}\right)$, the marginal likelihood of the model takes the form

$$
\begin{aligned}
L\left(\boldsymbol{\beta}, \boldsymbol{\theta}, \sigma_{u}^{2}\right) & =\prod_{i=1}^{N} \int_{u_{i}} \prod_{j=1}^{n_{i}}\left\{f_{i j}\left(t_{i j}\right)\right\}^{\delta_{i j}}\left\{S_{i j}\left(t_{i j}\right)\right\}^{1-\delta_{i j}} f\left(u_{i}\right) d u_{i} \\
& =\prod_{i=1}^{N} \int_{u_{i}} \prod_{j=1}^{n_{i}}\left\{h_{i j}\left(t_{i j}\right)\right\}^{\delta_{i j}} S_{i j}\left(t_{i j}\right) f\left(u_{i}\right) d u_{i} \\
& =\prod_{i=1}^{N} E_{u_{i}}\left\{\prod_{j=1}^{n_{i}} g\left(t_{i j} \mid u_{i}\right)\right\},
\end{aligned}
$$

where $g\left(t_{i j} \mid u_{i}\right)=\left\{h_{i j}\left(t_{i j}\right)\right\}^{\delta_{i j}} S_{i j}\left(t_{i j}\right)$, with $S_{i j}(t)$ being the survivor function for the $j^{\text {th }}$ event time for the $i^{t h}$ individual at time $t ; f_{i j}(t)$ being the corresponding density function at time $t$, and $E_{u_{i}}$ indicates the expectation with respect to the frailty $u_{i}$.

Similar to Sinha [26] (see also Cox [12]), a Taylor series expansion is used around $E_{u_{i}}=0$ (i.e. the mean of frailty) in order to expand the term $\prod_{j=1}^{n_{i}} g\left(t_{i j} \mid u_{i}\right)$ in the likelihood function (4.2) by taking the expectations with respect to $u_{i}$. The marginal likelihood for the $i^{\text {th }}$ individual may be expressed as 


$$
\begin{aligned}
L_{i}\left(\boldsymbol{\beta}, \boldsymbol{\theta}, \sigma_{u}^{2}\right) & =E_{u_{i}}\left\{\prod_{j=1}^{n_{i}} g\left(t_{i j} \mid u_{i}\right)\right\} \\
& =E_{u_{i}}\left\{g^{*}\left(t_{i} \mid u_{i}\right)\right\} \\
& =E_{u_{i}}\left\{g^{*}\left(t_{i} \mid u_{i}=0\right)+\left.\frac{\partial g^{*}\left(t_{i} \mid u_{i}\right)}{\partial u_{i}}\right|_{u_{i}=0}\left(u_{i}-0\right)+\left.\sum_{k=2}^{\infty} \frac{\partial^{k} g^{*}\left(t_{i} \mid u_{i}\right)}{u_{i}^{k}}\right|_{u_{i}=0} \frac{\left(u_{i}-0\right)^{k}}{k !}\right\} \\
& =\left\{g^{*}\left(t_{i} \mid u_{i}=0\right)+\left.\frac{\partial g^{*}\left(t_{i} \mid u_{i}\right)}{\partial u_{i}}\right|_{u_{i}=0} E\left(u_{i}\right)+\left.\sum_{k=2}^{\infty} \frac{\partial^{k} g^{*}\left(t_{i} \mid u_{i}\right)}{u_{i}^{k}}\right|_{u_{i}=0} \frac{E\left(u_{i}^{k}\right)}{k !}\right\} \\
& =\left\{g^{*}\left(t_{i} \mid u_{i}=0\right)+\sum_{k=2}^{\infty} \frac{a_{k}}{k !} \frac{\partial^{k} g^{*}\left(t_{i} \mid u_{i}\right)}{u_{i}^{k}}\right\},
\end{aligned}
$$

where

$g^{*}\left(t_{i} \mid u_{i}\right)=\prod_{j=1}^{n_{i}} g\left(t_{i j} \mid u_{i}\right), E\left(u_{i}\right)=0, E\left(u_{i}^{k}\right)=a_{k}$.

Then the logarithm of the marginal likelihood for the $i^{\text {th }}$ individuals becomes

$$
\log L_{i}\left(\boldsymbol{\beta}, \boldsymbol{\theta}, \sigma_{u}^{2}\right)=\log \left\{g^{*}\left(t_{i} \mid u_{i}=0\right)+\sum_{k=2}^{\infty} \frac{a_{k}}{k !} \frac{\partial^{k}}{\partial u_{i}^{k}} g^{*}\left(t_{i} \mid u_{i}\right)\right\}
$$

\subsubsection{Score Statistics}

In this section, we derive the score statistics for testing the null hypothesis $H_{0}: \sigma_{u}^{2}=0$ against the alternative hypothesis $H_{a}: \sigma_{u}^{2}>0$.

The score test is based on the score function of $\sigma_{u}^{2}$ evaluated at $\sigma_{u}^{2}=0$ :

$$
U(\boldsymbol{\gamma})=\left.\sum_{i=1}^{N} \frac{\partial \log L_{i}\left(\boldsymbol{\gamma}, \sigma_{u}^{2}\right)}{\partial \sigma_{u}^{2}}\right|_{\sigma_{u}^{2}=0}=\sum_{i=1}^{N} S_{i}^{*}(\boldsymbol{\gamma})
$$

where $\boldsymbol{\gamma}=\left(\boldsymbol{\beta}^{\prime}, \boldsymbol{\theta}^{\prime}\right)^{\prime}$ is the vector of unknown model parameters that needs to be estimated and $S_{i}^{*}(\gamma)$ is the score function of $\sigma_{u}^{2}$ for the $i^{t h}$ individual evaluated at the null hypothesis 
$H_{0}: \sigma_{u}^{2}=0$, given by

$$
\begin{aligned}
S_{i}^{*}(\boldsymbol{\gamma}) & =\left.\frac{\partial \log L_{i}\left(\boldsymbol{\beta}, \boldsymbol{\theta}, \sigma_{u}^{2}\right)}{\partial \sigma_{u}^{2}}\right|_{\sigma_{u}^{2}=0} \\
& =\left.\frac{1}{2}\left[\left\{\sum_{j=1}^{n_{i}} \frac{\partial}{\partial u_{i}} \log g\left(t_{i j} \mid u_{i}\right)\right\}^{2}+\sum_{j=1}^{n_{i}} \frac{\partial^{2}}{\partial u_{i}^{2}} \log g\left(t_{i j} \mid u_{i}\right)\right]\right|_{u_{i}=0} \\
& =\frac{1}{2}\left[\left\{\sum_{j=1}^{n_{i}} \delta_{i j}-\exp \left(\boldsymbol{x}_{i j}^{\prime} \boldsymbol{\beta}\right) H_{0}\left(t_{i j}\right)\right\}^{2}-\sum_{j=1}^{n_{i}} \exp \left(\boldsymbol{x}_{i j}^{\prime} \boldsymbol{\beta}\right) H_{0}\left(t_{i j}\right)\right],
\end{aligned}
$$

where $H_{0}\left(t_{i j}\right)$ is the baseline cumulative hazard function at time $t$ depending on a vector of unknown parameters $\boldsymbol{\theta}$ and $\boldsymbol{\beta}$ is a vector of unknown regression coefficients. The estimated variance of the score function $U(\gamma)$ is defined as $D(\hat{\gamma})=\left.D(\gamma)\right|_{\sigma_{u}^{2}=0}=I_{\sigma \sigma}-I_{\sigma \gamma} I_{\gamma \gamma}^{-1} I_{\gamma \sigma}$, which can be derived from the Fisher information matrix:

$$
I(\gamma)=\left(\begin{array}{cc}
I_{\gamma \gamma} & I_{\gamma \sigma} \\
I_{\sigma \gamma} & I_{\sigma \sigma}
\end{array}\right),
$$

where

$$
\begin{array}{r}
I_{\gamma \boldsymbol{\gamma}}=-\left.\sum_{i=1}^{N} \frac{\partial^{2} \log L_{i}\left(\boldsymbol{\gamma}, \sigma_{u}^{2}\right)}{\partial \boldsymbol{\gamma} \partial \boldsymbol{\gamma}^{\prime}}\right|_{\sigma_{u}^{2}=0}, \\
I_{\boldsymbol{\gamma} \boldsymbol{\sigma}}=I_{\boldsymbol{\sigma} \gamma}^{\prime}=-\left.\sum_{i=1}^{N} \frac{\partial^{2} \log L_{i}\left(\boldsymbol{\gamma}, \sigma_{u}^{2}\right)}{\partial \boldsymbol{\gamma} \partial \sigma_{u}^{2}}\right|_{\sigma_{u}^{2}=0},
\end{array}
$$

and

$$
I_{\boldsymbol{\sigma} \boldsymbol{\sigma}}=-\left.\sum_{i=1}^{N} \frac{\partial^{2} \log L_{i}\left(\boldsymbol{\gamma}, \sigma_{u}^{2}\right)}{\partial\left(\sigma_{u}^{2}\right)^{2}}\right|_{\sigma_{u}^{2}=0}
$$


Details on the derivation of the score function $U(\boldsymbol{\gamma})$ and Fisher information $I(\boldsymbol{\gamma})$ are given in Appendix 8.1.

After some algebra, we can show that

$$
\begin{aligned}
&\left.\frac{\partial^{2} \log L_{i}\left(\boldsymbol{\gamma}, \sigma_{u}^{2}\right)}{\partial \boldsymbol{\beta} \partial \boldsymbol{\beta}^{\prime}}\right|_{\sigma_{u}^{2}=0}=-\sum_{j=1}^{n_{i}} \boldsymbol{x}_{i j} \boldsymbol{x}_{i j}^{\prime} \exp \left(\boldsymbol{x}_{i j}^{\prime} \boldsymbol{\beta}\right) H_{0}\left(t_{i j}\right), \\
&\left.\frac{\partial^{2} \log L_{i}\left(\boldsymbol{\gamma}, \sigma_{u}^{2}\right)}{\partial \boldsymbol{\theta} \partial \boldsymbol{\beta}^{\prime}}\right|_{\sigma_{u}^{2}=0}=-\sum_{j=1}^{n_{i}} \frac{\partial H_{0}(t, \boldsymbol{\theta})}{\partial \boldsymbol{\theta}} \exp \left(\boldsymbol{x}_{i j}^{\prime} \boldsymbol{\beta}\right) \boldsymbol{x}_{i j}, \\
&\left.\frac{\partial^{2} \log L_{i}\left(\boldsymbol{\gamma}, \sigma_{u}^{2}\right)}{\partial \boldsymbol{\theta} \partial \boldsymbol{\theta}^{\prime}}\right|_{\sigma_{u}^{2}=0}=\sum_{j=1}^{n_{i}}\left[\delta_{i j} \frac{\partial^{2} \log h_{0}(t, \boldsymbol{\theta})}{\partial \boldsymbol{\theta} \partial \boldsymbol{\theta}^{\prime}}-\exp \left(\boldsymbol{x}_{i j}^{\prime} \boldsymbol{\beta}\right) \frac{\partial^{2} H_{0}\left(t_{i j}\right)}{\left.\partial \boldsymbol{\theta} \partial \boldsymbol{\theta}^{\prime}\right],}\right. \\
&\left.\frac{\partial^{2} \log L_{i}\left(\boldsymbol{\gamma}, \sigma_{u}^{2}\right)}{\partial \sigma_{u}^{2} \partial \boldsymbol{\beta}^{\prime}}\right|_{\sigma_{u}^{2}=0}=-\left\{\sum_{j=1}^{n_{i}} \boldsymbol{x}_{i j} \exp \left(\boldsymbol{x}_{i j}^{\prime} \boldsymbol{\beta}\right) H_{0}\left(t_{i j}\right)\right\}\left\{\sum_{j=1}^{n_{i}} \delta_{i j}-\sum_{j=1}^{n_{i}} \exp \left(\boldsymbol{x}_{i j}^{\prime} \boldsymbol{\beta}\right) H_{0}\left(t_{i j}\right)+\frac{1}{2}\right\}, \\
&\left.\frac{\partial^{2} \log L_{i}\left(\boldsymbol{\gamma}, \sigma_{u}^{2}\right)}{\partial \sigma_{u}^{2} \partial \boldsymbol{\theta}^{\prime}}\right|_{\sigma_{u}^{2}=0}=-\left\{\sum_{j=1}^{n_{i}} \frac{\partial H_{0}\left(t_{i j}\right)}{\partial \boldsymbol{\theta}} \exp \left(\boldsymbol{x}_{i j}^{\prime} \boldsymbol{\beta}\right)\right\}\left\{\sum_{j=1}^{n_{i}} \delta_{i j}-\sum_{j=1}^{n_{i}} H_{0}\left(t_{i j}\right) \exp \left(\boldsymbol{x}_{i j}^{\prime} \boldsymbol{\beta}\right)+\frac{1}{2}\right\},
\end{aligned}
$$

and

$$
\begin{aligned}
\left.\frac{\partial^{2} \log L_{i}\left(\boldsymbol{\gamma}, \sigma_{u}^{2}\right)}{\partial\left(\sigma_{u}^{2}\right)^{2}}\right|_{\sigma_{u}^{2}=0}= & -\left\{\sum_{j=1}^{n_{i}} H_{i j}\left(t_{i j}\right)\right\}\left\{\sum_{j=1}^{n_{i}} \delta_{i j}-\sum_{j=1}^{n_{i}} H_{i j}\left(t_{i j}\right)\right\}\left\{\sum_{j=1}^{n_{i}} \delta_{i j}-\sum_{j=1}^{n_{i}} H_{i j}\left(t_{i j}\right)+1\right\} \\
& \times \frac{1}{2}\left\{\sum_{j=1}^{n_{i}} H_{i j}\left(t_{i j}\right)\right\}-\frac{1}{4}\left\{\sum_{j=1}^{n_{i}} H_{i j}\left(t_{i j}\right)\right\},
\end{aligned}
$$

where $H_{i j}(t)=H_{0}(t) \exp \left(\boldsymbol{x}_{i j}^{\prime} \boldsymbol{\beta}\right)$.

Following Silvapulle and Silvapulle [24] and Sinha [26], the null hypothesis $H_{0}: \sigma_{u}^{2}=0$ against the one-sided alternative hypothesis $H_{a}: \sigma_{u}^{2}>0$ is tested by using a score test defined by

$$
T=\frac{U_{0}^{2}}{D_{0}}-\inf \left\{\frac{\left(U_{0}-\delta\right)^{2}}{D_{0}}: \delta>0\right\}
$$

As we notice, the score statistics $T$ is defined as a function of the score function $U_{0} \equiv U(\tilde{\gamma})$ and the variance function $D_{0} \equiv D(\tilde{\boldsymbol{\gamma}})$ with $\tilde{\boldsymbol{\gamma}}=\left(\tilde{\boldsymbol{\beta}}^{\prime}, \tilde{\boldsymbol{\theta}}^{\prime}\right)^{\prime}$ being the ML estimates of the model 
parameters $\boldsymbol{\gamma}=\left(\boldsymbol{\beta}^{\prime}, \boldsymbol{\theta}^{\prime}\right)^{\prime}$ under the null $H_{0}: \sigma_{u}^{2}=0$.

\subsubsection{Likelihood Function and Parameter Estimation}

\subsubsection{Under No Frailty $\left(\sigma_{u}^{2}=0\right)$}

Under $H_{0}: \sigma_{u}^{2}=0$, we have the standard proportional hazards model for the $i^{\text {th }}$ individual as

$$
h_{i}(t)=h_{0}(t) \exp \left(\boldsymbol{x}_{i}^{\prime} \boldsymbol{\beta}\right),
$$

where $h_{0}(t)$ is the baseline hazard function, $\boldsymbol{\beta}$ is a vector of unknown regression coefficients that needs to be estimated, and $\boldsymbol{x}_{i}$ is a vector of covariates for the $i^{t h}$ individual.

Then the likelihood function for the regression parameters is obtained as

$$
\begin{aligned}
L(\boldsymbol{\beta}) & =\prod_{i=1}^{N}\left\{f_{i}\left(t_{i} ; \boldsymbol{\beta}\right)\right\}^{\delta_{i}}\left\{S_{i}\left(t_{i} ; \boldsymbol{\beta}\right)\right\}^{1-\delta_{i}} \\
& =\prod_{i=1}^{N}\left\{\frac{f_{i}\left(t_{i} ; \boldsymbol{\beta}\right)}{S_{i}\left(t_{i} ; \boldsymbol{\beta}\right)}\right\}^{\delta_{i}} S_{i}\left(t_{i} ; \boldsymbol{\beta}\right) \\
& =\prod_{i=1}^{N}\left\{h_{i}\left(t_{i} ; \boldsymbol{\beta}\right)\right\}^{\delta_{i}} S_{i}\left(t_{i} ; \boldsymbol{\beta}\right),
\end{aligned}
$$

where $h_{i}\left(t_{i} ; \boldsymbol{\beta}\right), S_{i}\left(t_{i} ; \boldsymbol{\beta}\right)$ are the hazard rate and the survival function for the $i^{\text {th }}$ individual respectively, which depend on parameters $\boldsymbol{\beta}$, and $\delta_{i}$ is the censoring information $\left(\delta_{i}=0\right.$ if $T_{i}$ is right-censored; $\delta_{i}=1$ if $T_{i}$ is observed).

The likelihood function (4.9) can be derived using (4.8) as 


$$
\begin{aligned}
L(\boldsymbol{\beta}) & =\prod_{i=1}^{N}\left\{h_{0}(t) \exp \left(\boldsymbol{x}_{i}^{\prime} \boldsymbol{\beta}\right)\right\}^{\delta_{i}} e^{-\int_{0}^{t_{i}} h(y ; \boldsymbol{\beta}) d y} \\
& =\prod_{i=1}^{N}\left\{\lambda \exp \left(\boldsymbol{x}_{i}^{\prime} \boldsymbol{\beta}\right)\right\}^{\delta_{i}} e^{-\int_{0}^{t_{i}} \lambda \exp \left(\boldsymbol{x}_{i}^{\prime} \boldsymbol{\beta}\right) d y} \\
& =\prod_{i=1}^{N}\left\{\exp \left(\boldsymbol{x}_{i}^{\prime} \boldsymbol{\beta}\right)\right\}^{\delta_{i}} e^{-\exp \left(\boldsymbol{x}_{i}^{\prime} \boldsymbol{\beta}\right)} y_{i} \quad\left(h_{0}(t)=e^{\log \lambda}\right) .
\end{aligned}
$$

Taking logarithm of the likelihood function (4.10),

$$
\log L(\boldsymbol{\beta})=-\sum_{i=1}^{N} \exp \left(\boldsymbol{x}_{i}^{\prime} \boldsymbol{\beta}\right) y_{i}+\sum_{i=1}^{N} \delta_{i}\left(\boldsymbol{x}_{i}^{\prime} \boldsymbol{\beta}\right)
$$

The regression parameter $\boldsymbol{\beta}$ can be estimated by using the iterative Newton-Raphson method, as described in Harrell [16]. The iterative equations take the form

$$
\boldsymbol{\beta}^{(m+1)}=\boldsymbol{\beta}^{(m)}+\left(-\frac{\partial^{2} \log L(\boldsymbol{\beta})}{\partial \boldsymbol{\beta} \partial \boldsymbol{\beta}^{\prime}}\right)_{\boldsymbol{\beta}^{(m)}}^{-1}\left(\frac{\partial \log L(\boldsymbol{\beta})}{\partial \boldsymbol{\beta}}\right)_{\boldsymbol{\beta}^{(m)}},
$$

for $m=1,2, \ldots$,

where $U(\tilde{\boldsymbol{\beta}})=\left.\frac{\partial \log L(\boldsymbol{\beta})}{\partial \boldsymbol{\beta}}\right|_{\boldsymbol{\beta}=\boldsymbol{\beta}^{(m)}}$ is the score function of $\boldsymbol{\beta}$ evaluated at $\boldsymbol{\beta}=\boldsymbol{\beta}^{(m)}$,

and $I(\tilde{\boldsymbol{\beta}})=-\left.\frac{\partial^{2} \log L(\boldsymbol{\beta})}{\partial \boldsymbol{\beta} \partial \boldsymbol{\beta}^{t}}\right|_{\boldsymbol{\beta}=\boldsymbol{\beta}^{(m)}}$ is the observed Fisher information matrix evaluated at $\boldsymbol{\beta}=\boldsymbol{\beta}^{(m)}$.

The score function of regression coefficients $\boldsymbol{\beta}$ for all individuals is obtained as

$$
U(\tilde{\boldsymbol{\beta}})=\frac{\partial \log L(\boldsymbol{\beta})}{\partial \boldsymbol{\beta}}=-\sum_{i=1}^{N} \exp \left(\boldsymbol{x}_{i}^{\prime} \boldsymbol{\beta}\right) \boldsymbol{x}_{i} y_{i}+\sum_{i=1}^{N} \delta_{i}\left(\boldsymbol{x}_{i}^{\prime} \boldsymbol{\beta}\right)
$$

and the observed Fisher information matrix is obtained from 


$$
D(\tilde{\boldsymbol{\beta}})=\frac{\partial^{2} \log L(\boldsymbol{\beta})}{\partial \boldsymbol{\beta} \partial \boldsymbol{\beta}^{\prime}}=-\sum_{i=1}^{N} \exp \left(\boldsymbol{x}_{i}^{\prime} \boldsymbol{\beta}\right) \boldsymbol{x}_{i} \boldsymbol{x}_{i}^{\prime} y_{i} .
$$

The asymptotic variance of the ML estimator of $\boldsymbol{\beta}$ is obtained from the inverse of the Fisher information of $\boldsymbol{\beta}$.

\subsubsection{Under Frailty $\left(\sigma_{u}^{2} \neq 0\right)$}

In case the null hypothesis $H_{0}: \sigma_{u}^{2}=0$ is rejected, the proportional hazards model with frailty (4.1) is fitted, and the maximum likelihood estimates of this model are obtained.

The likelihood function for given data $\left(T_{i}, \delta_{i}, \boldsymbol{x}_{\boldsymbol{i}}, u_{i}\right)(i=1,2, \ldots, N)$ as shown in (4.2) is used to obtain the maximum likelihood estimates. We use the R function "optim" for the maximum likelihood (ML) estimates of the regression coefficients and variance component in the frailty model. 


\section{Chapter 5}

\section{Simulation Study}

\subsection{Generated Clustered Data}

In this chapter, we ran a series of simulations to study the finite-sample properties of the onesided score test based on the mixture of chi-square distributions for testing the significance of the variance component in a frailty model. We investigate the empirical level and power of the score test for a shared frailty model under both correctly specified random effects (i.e., $u_{i} \sim$ $\left.N\left(0, \sigma_{u}^{2}\right)\right)$ and misspecified random effects $\left(i . e ., u_{i}=\sigma_{u}\left(\frac{a_{i}-\lambda}{\sqrt{\lambda}}\right)\right.$, with $\left.a_{i} \sim G a(\lambda, 1)\right)$. We also study empirical biases and mean squared errors of the regression estimators under correctly specified and misspecified proportional hazards models.

The survival data were generated from the hazards model

$$
h_{i j}(t)=h_{0}(t) \exp \left(\beta_{1} x_{i j 1}+\beta_{2} x_{i j 2}+u_{i}\right), i=1, \ldots ., N ; j=1, \ldots \ldots, n,
$$

where $u_{i}$ is $N\left(0, \sigma_{u}^{2}\right)$ under the correctly specified model and $u_{i}=\sigma_{u}\left(\frac{a_{i}-\lambda}{\sqrt{\lambda}}\right)$, with $a_{i} \sim$ $G a(\lambda, 1)$ under the misspecified model.

For the given model, each subject $j$ within a cluster $i$ has two covariates, that is, $\boldsymbol{x}_{i j}=$ $\left(x_{i j 1}, x_{i j 2}\right)^{\prime}$, where $x_{i j 1}$ was distributed as uniform(-1,1), and $x_{i j 2}$ was distributed as $N(0,1)$. We assume a parametric form of the baseline hazard $h_{o}(t)$, which is an exponential with a 
positive parameter $\lambda$, and is a special case of Weibull distribution when the shape parameter $\gamma=1$

Model (5.1) can be written in the form

$$
\begin{aligned}
h_{i j}(t) & =\lambda \exp \left(\beta_{1} x_{i j 1}+\beta_{2} x_{i j 2}+u_{i}\right) \\
& =\exp \left(\beta_{0}+\beta_{1} x_{i j 1}+\beta_{1} x_{i j 2}+u_{i}\right), i=1, \ldots, N ; j=1, \ldots \ldots, n,
\end{aligned}
$$

where $\beta_{0}=\log \lambda, \boldsymbol{x}_{i j}=\left(1, x_{i j 1}, x_{i j 2}\right)^{\prime}$ represents a vector of covariates, and $\boldsymbol{\beta}=\left(\beta_{0}, \beta_{1}, \beta_{2}\right)^{\prime}$ represents a vector of unknown regression coefficients.

Survival times $\left(T_{i 1}, \ldots, T_{i n_{i}}\right)$ in a cluster $i$ with frailties $u_{i}$ were generated independently from the conditional hazard $h_{i j}(t)$, where the values of the regression parameters $\left(\beta_{0}, \beta_{1}, \beta_{2}\right)$ were fixed at $(-2.53,0.5,-0.25)$.

Also, the censoring times $C_{i j}$ were assumed to be independently and identically distributed random variables generated from exponential distribution with mean $\frac{1}{\lambda}=\frac{1}{0.08}$. We ran a series of simulations based on 1000 replicates of data sets for each combination of $N$ $=100,200,300$ clusters and $n=2,5,10,40$ subjects within a cluster $i$.

The random effects $u_{i}$ were modelled in two ways: (1) In case of the correctly specified model, we assumed frailties $u_{i}$ to be independently and normally distributed with mean zero and variance component $\sigma_{u}^{2} ;(2)$ In case of the misspecified model, $u_{i}$ were generated from $u_{i}=\sigma_{u}\left(\frac{a_{i}-\lambda}{\sqrt{\lambda}}\right)$, where $a_{i} \sim \operatorname{Gamma}(\lambda, 1)$.

Note that the mean and variance of the frailty terms are $E\left(u_{i}\right)=0$ and $\operatorname{var}\left(u_{i}\right)=\sigma_{u}^{2}$ respectively, which are the same under both correctly specified and misspecified models.

The observed data are $\left(t_{i j}, \delta_{i j}, \boldsymbol{x}_{i j}\right)$, where $\delta_{i j}$ is the censoring indicator for the $j^{\text {th }}$ survival time in the $i^{\text {th }}$ cluster. 


\subsection{Empirical Level and Power of the Score Test for Shared Frialty}

To begin with, the significance level of a test, also denoted as $\alpha$, is known as the probability of rejecting the null hypothesis when it is true. The empirical level of the score test that is based on the asymptotic mixture of chi-square distributions is calculated by carrying out a simulation study using the generated data from the exponential proportional hazards model with no frailty. The data were generated under the null hypothesis $H_{0}: \sigma_{u}^{2}=0$ for different sample sizes. The empirical level is obtained as the proportion of times the null hypothesis $H_{0}$ is rejected, when, in fact, $H_{0}$ is true.

Moreover, we investigate the empirical power of the score test when data were generated from the exponential proportional hazards model with shared frailty. The powers were calculated under different values of $\sigma_{u}^{2}=(0.05,0.1,0.2)$.

\subsubsection{Correctly Specified Random Effects Distribution}

In this case, we assume a normal distribution for the random effects when studying the properties of the score test. We performed a simulation study based on 1000 replicates of data sets in which the random effects $u_{i}$ (frailties) follow a normal distribution with mean 0 and variance component $\sigma_{u}^{2}$.

Table 5.1 shows empirical level and powers of the score test under different sample sizes. As we can notice from the results, the level of the proposed test gets generally closer to the nominal 0.05 level when the number of clusters $(N)$ as well as the cluster size $(n)$ increase, as expected.

For studying the powers of the score test, we used the same simulation configurations as explained above. Table 5.1 presents empirical powers of the score test for each combination 
Table 5.1: Empirical level and power of the score test for shared frailty model under correctly specified random effects: $u_{i} \sim N\left(0, \sigma_{u}^{2}\right)$.

\begin{tabular}{cccccc}
\hline \hline & number of & \multicolumn{4}{c}{ cluster size(n) } \\
\cline { 4 - 6 }$\sigma_{u}^{2}$ & clusters (N) & 2 & 5 & 10 & 40 \\
\hline \hline \multirow{2}{*}{0} & 100 & 0.008 & 0.018 & 0.027 & 0.027 \\
& 200 & 0.015 & 0.023 & 0.028 & 0.023 \\
& 300 & 0.015 & 0.026 & 0.027 & 0.041 \\
0.05 & 100 & 0.029 & 0.105 & 0.405 & 1.000 \\
& 200 & 0.07 & 0.266 & 0.726 & 1.000 \\
& 300 & 0.083 & 0.400 & 0.880 & 1.000 \\
0.1 & 100 & 0.055 & 0.351 & 0.880 & 1.000 \\
& 200 & 0.180 & 0.676 & 0.997 & 1.000 \\
& 300 & 0.222 & 0.854 & 0.998 & 1.000 \\
0.2 & 100 & 0.201 & 0.852 & 0.999 & 1.000 \\
& 200 & 0.447 & 0.989 & 1.000 & 1.000 \\
& 300 & 0.620 & 0.998 & 1.000 & 1.000 \\
\hline \hline
\end{tabular}

of $\sigma_{u}^{2}=(0.05,0.1,0.2)$. As expected, the power increases with the increased values of $N$ and $n$.

\subsubsection{Misspecified Random Effects Distribution}

In practice, the normality assumption for the random effects may be violated in some situations. So we examine the properties of the score test under misspecified ,non-normal random effects. Tables 5.2 and 5.3 present the empirical level and power of the score test for testing $H_{0}: \sigma_{u}^{2}=0$ vs $H_{0}: \sigma_{u}^{2}>0$ under misspecified random effects: $u_{i}=\sigma_{u}\left(\frac{a_{i}-\lambda}{\sqrt{\lambda}}\right)$, with $a_{i} \sim \operatorname{Gamma}(\lambda, 1)$. Similar to the case of normal random effects $u_{i}$, we generated $S=1000$ replicates of data sets from the exponential hazards model with shared frailty, but the frailties $u_{i}$ were obtained from the gamma distribution. The empirical level of the test is calculated under the null hypothesis $H_{0}: \sigma_{u}^{2}=0$, while the empirical power of the test is calculated under the alternative $H_{0}: \sigma_{u}^{2}>0$ for $\sigma_{u}^{2}=(0.05,0.1,0.2)$ and $\lambda=(0.5,1,2,4)$. 
Table 5.2: Empirical level and power of the score test for shared frailty model under misspecified random effects: $u_{i}=\sigma_{u}\left(\frac{a_{i}-\lambda}{\sqrt{\lambda}}\right)$, where $a_{i} \sim G a(\lambda, 1)$ and $\lambda=0.5,1$.

\begin{tabular}{|c|c|c|c|c|c|c|}
\hline \multirow[b]{2}{*}{$\lambda$} & \multirow[b]{2}{*}{$\sigma_{u}^{2}$} & \multirow{2}{*}{$\begin{array}{c}\text { number of } \\
\text { clusters }(\mathrm{N})\end{array}$} & \multicolumn{4}{|c|}{ cluster size $(\mathrm{n})$} \\
\hline & & & 2 & 5 & 10 & 40 \\
\hline \multirow[t]{12}{*}{0.5} & 0 & 100 & 0.007 & 0.011 & 0.031 & 0.030 \\
\hline & & 200 & 0.017 & 0.020 & 0.021 & 0.037 \\
\hline & & 300 & 0.012 & 0.025 & 0.022 & 0.036 \\
\hline & 0.05 & 100 & 0.033 & 0.120 & 0.354 & 0.991 \\
\hline & & 200 & 0.064 & 0.235 & 0.641 & 1.000 \\
\hline & & 300 & 0.065 & 0.306 & 0.798 & 1.000 \\
\hline & 0.1 & 100 & 0.051 & 0.311 & 0.769 & 1.000 \\
\hline & & 200 & 0.120 & 0.571 & 0.963 & 1.000 \\
\hline & & 300 & 0.157 & 0.738 & 0.997 & 1.000 \\
\hline & 0.2 & 100 & 0.142 & 0.660 & 0.972 & 1.000 \\
\hline & & 200 & 0.284 & 0.931 & 1.000 & 1.000 \\
\hline & & 300 & 0.462 & 0.987 & 1.000 & 1.000 \\
\hline \multirow[t]{12}{*}{1} & 0 & 100 & 0.013 & 0.012 & 0.026 & 0.018 \\
\hline & & 200 & 0.023 & 0.030 & 0.026 & 0.032 \\
\hline & & 300 & 0.020 & 0.030 & 0.034 & 0.034 \\
\hline & 0.05 & 100 & 0.028 & 0.109 & 0.376 & 0.993 \\
\hline & & 200 & 0.051 & 0.252 & 0.644 & 1.000 \\
\hline & & 300 & 0.066 & 0.350 & 0.838 & 1.000 \\
\hline & 0.1 & 100 & 0.063 & 0.336 & 0.812 & 1.000 \\
\hline & & 200 & 0.121 & 0.613 & 0.981 & 1.000 \\
\hline & & 300 & 0.209 & 0.798 & 0.998 & 1.000 \\
\hline & 0.2 & 100 & 0.175 & 0.748 & 0.990 & 1.000 \\
\hline & & 200 & 0.356 & 0.962 & 1.000 & 1.000 \\
\hline & & 300 & 0.535 & 0.998 & 1.000 & 1.000 \\
\hline
\end{tabular}


Table 5.3: Empirical level and power of the score test for shared frailty model under misspecified random effects: $u_{i}=\sigma_{u}\left(\frac{a_{i}-\lambda}{\sqrt{\lambda}}\right)$, where $a_{i} \sim G a(\lambda, 1)$ and $\lambda=2,4$.

\begin{tabular}{|c|c|c|c|c|c|c|}
\hline \multirow[b]{2}{*}{$\lambda$} & \multirow[b]{2}{*}{$\sigma_{u}^{2}$} & \multirow{2}{*}{$\begin{array}{c}\text { number of } \\
\text { clusters }(\mathrm{N})\end{array}$} & \multicolumn{4}{|c|}{ "cluster size(n) } \\
\hline & & & 2 & 5 & 10 & 40 \\
\hline \multirow[t]{12}{*}{2} & \multirow[t]{3}{*}{0} & 100 & 0.010 & 0.019 & 0.019 & 0.020 \\
\hline & & 200 & 0.018 & 0.017 & 0.020 & 0.026 \\
\hline & & 300 & 0.022 & 0.030 & 0.024 & 0.033 \\
\hline & \multirow[t]{3}{*}{0.05} & 100 & 0.031 & 0.118 & 0.377 & 0.998 \\
\hline & & 200 & 0.050 & 0.232 & 0.674 & 1.000 \\
\hline & & 300 & 0.083 & 0.354 & 0.858 & 1.000 \\
\hline & \multirow[t]{3}{*}{0.1} & 100 & 0.070 & 0.339 & 0.817 & 1.000 \\
\hline & & 200 & 0.139 & 0.652 & 0.987 & 1.000 \\
\hline & & 300 & 0.197 & 0.808 & 1.000 & 1.000 \\
\hline & \multirow[t]{3}{*}{0.2} & 100 & 0.181 & 0.796 & 0.995 & 1.000 \\
\hline & & 200 & 0.422 & 0.978 & 1.000 & 1.000 \\
\hline & & 300 & 0.590 & 0.996 & 1.000 & 1.000 \\
\hline \multirow[t]{12}{*}{4} & \multirow[t]{3}{*}{0} & 100 & 0.012 & 0.021 & 0.017 & 0.021 \\
\hline & & 200 & 0.019 & 0.022 & 0.028 & 0.016 \\
\hline & & 300 & 0.019 & 0.025 & 0.024 & 0.033 \\
\hline & \multirow[t]{3}{*}{0.05} & 100 & 0.024 & 0.134 & 0.412 & 0.999 \\
\hline & & 200 & 0.051 & 0.273 & 0.700 & 1.000 \\
\hline & & 300 & 0.088 & 0.388 & 0.843 & 1.000 \\
\hline & \multirow[t]{3}{*}{0.1} & 100 & 0.053 & 0.359 & 0.844 & 1.000 \\
\hline & & 200 & 0.133 & 0.659 & 0.990 & 1.000 \\
\hline & & 300 & 0.226 & 0.836 & 0.999 & 1.000 \\
\hline & \multirow[t]{3}{*}{0.2} & 100 & 0.168 & 0.809 & 0.998 & 1.000 \\
\hline & & 200 & 0.42 & 0.988 & 1.000 & 1.000 \\
\hline & & 300 & 0.603 & 1.000 & 1.000 & 1.000 \\
\hline
\end{tabular}


Here also we observe that when the sample size is small, the empirical levels are generally lower than nominal 5\% level. But they get closer to the nominal 5\% level when the cluster size $n$ and number of clusters $N$ increase.

In summary, it appears from the results that in case of misspecified random effects, the empirical level and power are not significantly different than those obtained under correctly specified random effects. So we can conclude that the score test is not much affected by this misspecification.

\subsection{Empirical Biases and Mean Squared Errors}

For estimating the regression and heterogeneity parameters, we study the biases and mean squared errors (MSEs) of the estimators for model parameters (5.2) under both correctly specified random effects (i.e., $u_{i} \sim N\left(0, \sigma_{u}^{2}\right)$ ) and misspecified random effects (i.e., $u_{i}=$ $\sigma_{u}\left(\frac{a_{i}-\lambda}{\sqrt{\lambda}}\right)$, with $\left.a_{i} \sim G a(\lambda, 1)\right)$.

\section{Bias:}

By definition, the bias of an estimator $\hat{\theta}$ of a parameter $\theta$ is calculated as the difference between the expected value of $\hat{\theta}$ and the parameter $\theta$, given by

$$
\begin{aligned}
\operatorname{Bias}(\hat{\theta}) & =E(\hat{\theta})-\theta \\
& \cong\left\{\frac{1}{S} \sum_{i=1}^{S} \hat{\theta}^{(s)}\right\}-\theta
\end{aligned}
$$

where $\hat{\theta}^{(s)}$ is the estimator of $\theta$ obtained at the $s^{\text {th }}$ simulation.

\section{Mean Squared Error:}

The mean squared error $(M S E)$ of an estimator $\hat{\theta}$ of a parameter $\theta$ can be obtained 
from set of simulations as

$$
\begin{aligned}
\operatorname{MSE}(\hat{\theta}) & =E(\hat{\theta}-\theta)^{2} \\
& \cong \frac{1}{S} \sum_{i=1}^{S}\left(\hat{\theta}^{(s)}-\theta\right)^{2}
\end{aligned}
$$

where $\hat{\theta}^{(s)}$ is the estimator of $\theta$ obtained at the $s^{\text {th }}$ simulation.

Using the same generated data sets explained in section (5.1), we ran a series of simulations based on 1000 replicates of data sets for each combination of $N=100,200,300$ clusters with cluster size $n=25,10,40$. For each set of simulation, we study the biases and mean squared errors of the estimators of regression parameters $\left(\beta_{0}, \beta_{1}, \beta_{2}\right)$ under a misspecified model with no frailty when the correct model is (5.2) with frailty.

We also study the empirical properties of the estimators under correctly specified models with frailty. Details are given in the next section.

\subsubsection{True Model: Frailty with $u_{i} \sim N\left(0, \sigma_{u}^{2}\right)$}

\subsubsection{Fitted Model: No Frailty}

It is apparent from Tables 5.4 - 5.5 that the MSEs of the estimators of all parameters $\left(\beta_{0}, \beta_{1}, \beta_{2}\right)$ increase when the frailty variance component $\sigma_{u}^{2}$ increases. Also, the MSEs generally increase as the number of clusters $(\mathrm{N})$ and the cluster size (n) increase.

The biases of the estimators of the regression parameters generally increase as $\sigma_{u}^{2}$ increases. When $\sigma_{u}^{2}=4$ in Table 5.5, for instance, the biases of the estimates of the regression parameters $\left(\beta_{0}, \beta_{1}, \beta_{2}\right)$ with the number of clusters and the cluster size $(\mathrm{N}=100, \mathrm{n}=40)$ are $-0.111880,-0.19288$, and 0.09629 respectively, while the biases of the estimates of the same regression parameters are $0.00108,0.00125$, and -0.00020 respectively, when $\sigma_{u}^{2}=0$ (see Table 5.4).

In general, the simulation shows that the data with no frailty variance component $\left(\sigma_{u}^{2}\right)$ 
Table 5.4: Empirical biases and mean squared errors (MSEs) of the estimators of the regression parameters $\left(\beta_{0}, \beta_{1}, \beta_{2}\right)$ under misspecified model with no frailty, when the true model is frailty with $u_{i} \sim N\left(0, \sigma_{u}^{2}\right)$ and $\sigma_{u}^{2}=0,0.5$. Regression parameters are fixed at $\left(\beta_{0}, \beta_{1}, \beta_{2}\right)=(-2.53,0.5,-0.25)$.

\begin{tabular}{|c|c|c|c|c|c|c|c|c|}
\hline \multirow[b]{2}{*}{$\sigma_{u}^{2}$} & \multirow{2}{*}{$\begin{array}{c}\text { number of } \\
\text { clusters }\end{array}$} & \multirow{2}{*}{$\begin{array}{c}\text { cluster } \\
\text { size }\end{array}$} & \multicolumn{3}{|c|}{ Bias } & \multicolumn{3}{|c|}{ MSE } \\
\hline & & & $\beta_{0}$ & $\beta_{1}$ & $\beta_{2}$ & $\beta_{0}$ & $\beta_{1}$ & $\beta_{2}$ \\
\hline \multirow[t]{12}{*}{0} & 100 & 2 & 0.00177 & 0.00546 & -0.00081 & 0.00909 & 0.02828 & 0.01035 \\
\hline & & 5 & -0.00104 & -0.00155 & 0.00051 & 0.00370 & 0.01020 & 0.00375 \\
\hline & & 10 & 0.00122 & -0.00131 & 0.00217 & 0.00172 & 0.00531 & 0.00183 \\
\hline & & 40 & 0.00108 & 0.00125 & -0.00020 & 0.00040 & 0.00131 & 0.00046 \\
\hline & 200 & 2 & 0.00414 & -0.00117 & -0.00093 & 0.00460 & 0.01333 & 0.00469 \\
\hline & & 5 & 0.00012 & 0.00152 & -0.00100 & 0.00185 & 0.00567 & 0.00174 \\
\hline & & 10 & -0.00061 & 0.00030 & -0.00067 & 0.00083 & 0.00269 & 0.00088 \\
\hline & & 40 & -0.00015 & 0.00085 & 0.00007 & 0.00022 & 0.00067 & 0.00021 \\
\hline & 300 & 2 & 0.00010 & 0.00238 & 0.00154 & 0.00287 & 0.00919 & 0.00317 \\
\hline & & 5 & 0.00155 & -0.00106 & -0.00165 & 0.00121 & 0.00363 & 0.00122 \\
\hline & & 10 & -0.00006 & -0.00163 & -0.00235 & 0.00056 & 0.00195 & 0.00058 \\
\hline & & 40 & 0.00013 & 0.00011 & -0.00075 & 0.00015 & 0.00043 & 0.00014 \\
\hline \multirow[t]{12}{*}{0.5} & 100 & 2 & -0.02355 & -0.05321 & 0.01800 & 0.01443 & 0.03984 & 0.01246 \\
\hline & & 5 & -0.02105 & -0.04506 & 0.02288 & 0.00843 & 0.01538 & 0.00526 \\
\hline & & 10 & -0.03004 & -0.04163 & 0.02160 & 0.00658 & 0.00859 & 0.00266 \\
\hline & & 40 & -0.02662 & -0.04968 & 0.02426 & 0.00499 & 0.00425 & 0.00113 \\
\hline & 200 & 2 & -0.02603 & -0.04820 & 0.02595 & 0.00773 & 0.02073 & 0.00680 \\
\hline & & 5 & -0.02762 & -0.04590 & 0.02278 & 0.00484 & 0.00916 & 0.00291 \\
\hline & & 10 & -0.02779 & -0.04641 & 0.02346 & 0.00394 & 0.00561 & 0.00166 \\
\hline & & 40 & -0.02597 & -0.05003 & 0.02446 & 0.00299 & 0.00342 & 0.00090 \\
\hline & 300 & 2 & -0.02421 & -0.05019 & 0.02720 & 0.00465 & 0.01377 & 0.00442 \\
\hline & & 5 & -0.02797 & -0.04826 & 0.02738 & 0.00347 & 0.00715 & 0.00214 \\
\hline & & 10 & -0.02958 & -0.04586 & 0.02274 & 0.00299 & 0.00445 & 0.00128 \\
\hline & & 40 & -0.02943 & -0.04827 & 0.02430 & 0.00241 & 0.00295 & 0.00079 \\
\hline
\end{tabular}


Table 5.5: Empirical biases and mean squared errors (MSEs) of the estimators of the regression parameters $\left(\beta_{0}, \beta_{1}, \beta_{2}\right)$ under misspecified model with no frailty, when the true model is frailty with $u_{i} \sim N\left(0, \sigma_{u}^{2}\right)$ and $\sigma_{u}^{2}=1,4$. Regression parameters are fixed at $\left(\beta_{0}, \beta_{1}, \beta_{2}\right)=(-2.53,0.5,-0.25)$.

\begin{tabular}{|c|c|c|c|c|c|c|c|c|}
\hline \multirow[b]{2}{*}{$\sigma_{u}^{2}$} & \multirow{2}{*}{$\begin{array}{c}\text { number of } \\
\text { clusters }\end{array}$} & \multirow{2}{*}{$\begin{array}{l}\text { cluster } \\
\text { size }\end{array}$} & \multicolumn{3}{|c|}{ Bias } & \multicolumn{3}{|c|}{ MSE } \\
\hline & & & $\beta_{0}$ & $\beta_{1}$ & $\beta_{2}$ & $\beta_{0}$ & $\beta_{1}$ & $\beta_{2}$ \\
\hline \multirow[t]{12}{*}{1} & 100 & 2 & -0.03864 & -0.07118 & 0.03579 & 0.01880 & 0.04467 & 0.01590 \\
\hline & & 5 & -0.04950 & -0.08061 & 0.03805 & 0.01344 & 0.02292 & 0.00731 \\
\hline & & 10 & -0.04628 & -0.08169 & 0.04492 & 0.01072 & 0.01420 & 0.00456 \\
\hline & & 40 & -0.05049 & -0.08236 & 0.04130 & 0.01010 & 0.00896 & 0.00239 \\
\hline & 200 & 2 & -0.04782 & -0.08598 & 0.03538 & 0.01067 & 0.02854 & 0.00811 \\
\hline & & 5 & -0.04956 & -0.08855 & 0.04315 & 0.00867 & 0.01559 & 0.00458 \\
\hline & & 10 & -0.04948 & -0.09042 & 0.03973 & 0.00665 & 0.01238 & 0.00292 \\
\hline & & 40 & -0.04586 & -0.08585 & 0.04156 & 0.00586 & 0.00842 & 0.00204 \\
\hline & 300 & 2 & -0.04791 & -0.07945 & 0.04409 & 0.00758 & 0.01947 & 0.00638 \\
\hline & & 5 & -0.05009 & -0.08645 & 0.04268 & 0.00626 & 0.01288 & 0.00360 \\
\hline & & 10 & -0.04857 & -0.08472 & 0.04183 & 0.00527 & 0.00994 & 0.00262 \\
\hline & & 40 & -0.04949 & -0.08474 & 0.04248 & 0.00499 & 0.00786 & 0.00205 \\
\hline \multirow[t]{12}{*}{4} & 100 & 2 & -0.10740 & -0.18404 & 0.08149 & 0.03737 & 0.09068 & 0.02761 \\
\hline & & 5 & -0.10589 & -0.19210 & 0.09715 & 0.03172 & 0.05854 & 0.01692 \\
\hline & & 10 & -0.10333 & -0.19061 & 0.09247 & 0.02956 & 0.04733 & 0.01247 \\
\hline & & 40 & -0.11180 & -0.19288 & 0.09629 & 0.02948 & 0.04012 & 0.01025 \\
\hline & 200 & 2 & -0.11080 & -0.18836 & 0.09912 & 0.02675 & 0.06296 & 0.02017 \\
\hline & & 5 & -0.11611 & -0.19370 & 0.09708 & 0.02353 & 0.04792 & 0.01313 \\
\hline & & 10 & -0.11771 & -0.19364 & 0.09780 & 0.02323 & 0.04301 & 0.01138 \\
\hline & & 40 & -0.10989 & -0.19507 & 0.09620 & 0.01964 & 0.03952 & 0.00971 \\
\hline & 300 & 2 & -0.11641 & -0.19505 & 0.09464 & 0.02282 & 0.05498 & 0.01547 \\
\hline & & 5 & -0.11389 & -0.19736 & 0.09475 & 0.01950 & 0.04595 & 0.01144 \\
\hline & & 10 & -0.11297 & -0.19760 & 0.09848 & 0.01889 & 0.04294 & 0.01091 \\
\hline & & 40 & -0.11167 & -0.19791 & 0.09718 & 0.01788 & 0.04017 & 0.00978 \\
\hline
\end{tabular}


provides estimates with lower biases, but the biases increase as $\sigma_{u}^{2}$ increases.

\subsubsection{Fitted Model: Frailty with $u_{i} \sim N\left(0, \sigma_{u}^{2}\right)$}

In this case, we study the empirical biases and MSEs of the estimators of both the regression parameters $\left(\beta_{0}, \beta_{1}, \beta_{2}\right)$ and frailty variance component $\sigma_{u}^{2}$ under correctly specified models.

It is clear from Tables 5.6 - 5.8 that the frailty model generally provides unbiased estimates of both the regression parameters and variance component. For example, at $\sigma_{u}^{2}=4$ we see from Table 5.8 that when $N=100$ and $n=10$, the biases of estimates of $\left(\beta_{0}, \beta_{1}, \beta_{2}, \sigma_{u}^{2}\right)$ are $(-0.01399,-0.00283,0.00303,0.00271)$. The biases tend to be negligible for large samples, as expected.

For the MSEs of the estimators of model parameters with shared frailty, the empirical study shows that the MSEs increase as $\sigma_{u}^{2}$ increases. But these tend to be small when there is an increase in the number of clusters $(N)$ and cluster size $(n)$. For instance, Figures 5.15.6 clearly show the increase in the mean squared errors for the estimators of the regression parameters $\left(\beta_{0}, \beta_{1}, \beta_{2}\right)$ and the frailty variance component $\left(\sigma_{u}^{2}\right)$ as $\sigma_{u}^{2}$ increases from 0.5 to 4. But the MSEs tend to decrease when the number of clusters $(N)$ and cluster size $(n)$ increase. 
Table 5.6: Empirical biases and mean squared errors $(M S E s)$ of the estimators of both the regression parameters $\left(\beta_{0}, \beta_{1}, \beta_{2}\right)$ and the frailty variance component $\left(\sigma_{u}^{2}\right)$ under the correct model with frailty, when the random effects are correctly specified: $u_{i} \sim$ $N\left(0, \sigma_{u}^{2}\right)$ where $\sigma_{u}^{2}=0.5$. Regression parameters and variance component are fixed at $\left(\beta_{0}, \beta_{1}, \beta_{2}, \sigma_{u}^{2}\right)=(-2.53,0.5,-0.25,0.5)$.

\begin{tabular}{|c|c|c|c|c|c|c|c|c|c|c|}
\hline \multirow[b]{2}{*}{$\sigma_{u}^{2}$} & \multirow{2}{*}{$\begin{array}{c}\text { number of } \\
\text { clusters }\end{array}$} & \multirow{2}{*}{$\begin{array}{c}\text { cluster } \\
\text { size }\end{array}$} & \multicolumn{4}{|c|}{ Bias } & \multicolumn{4}{|c|}{ MSE } \\
\hline & & & $\beta_{0}$ & $\beta_{1}$ & $\beta_{2}$ & $\sigma_{u}^{2}$ & $\beta_{0}$ & $\beta_{1}$ & $\beta_{2}$ & $\sigma_{u}^{2}$ \\
\hline \multirow[t]{12}{*}{0.5} & 100 & 2 & -0.00231 & 0.00416 & 0.00065 & -0.01782 & 0.01615 & 0.04067 & 0.01291 & 0.05830 \\
\hline & & 5 & -0.00301 & -0.00130 & -0.00173 & -0.00192 & 0.00971 & 0.01407 & 0.00475 & 0.01813 \\
\hline & & 10 & 0.00091 & 0.00088 & -0.00027 & -0.00695 & 0.00698 & 0.00620 & 0.00206 & 0.00971 \\
\hline & & 40 & 0.00258 & -0.00056 & 0.00004 & -0.00592 & 0.00555 & 0.00139 & 0.00047 & 0.00640 \\
\hline & 200 & 2 & -0.00089 & 0.00035 & 0.00373 & 0.00445 & 0.00751 & 0.01864 & 0.00593 & 0.02780 \\
\hline & & 5 & -0.00278 & -0.00193 & -0.00010 & 0.00231 & 0.00461 & 0.00654 & 0.00241 & 0.00881 \\
\hline & & 10 & -0.00069 & 0.00088 & 0.00025 & 0.00119 & 0.00333 & 0.00304 & 0.00111 & 0.00516 \\
\hline & & 40 & 0.00090 & -0.00040 & -0.00017 & -0.00041 & 0.00271 & 0.00071 & 0.00023 & 0.00309 \\
\hline & 300 & 2 & -0.00139 & -0.00192 & -0.00223 & -0.00028 & 0.00519 & 0.01210 & 0.00430 & 0.02133 \\
\hline & & 5 & -0.00052 & 0.00345 & 0.00035 & -0.00112 & 0.00292 & 0.00462 & 0.00144 & 0.00602 \\
\hline & & 10 & -0.00002 & -0.00362 & -0.00001 & -0.00652 & 0.00249 & 0.00225 & 0.00072 & 0.00365 \\
\hline & & 40 & -0.00038 & -0.00118 & 0.00046 & -0.00277 & 0.00180 & 0.00045 & 0.00016 & 0.00204 \\
\hline
\end{tabular}




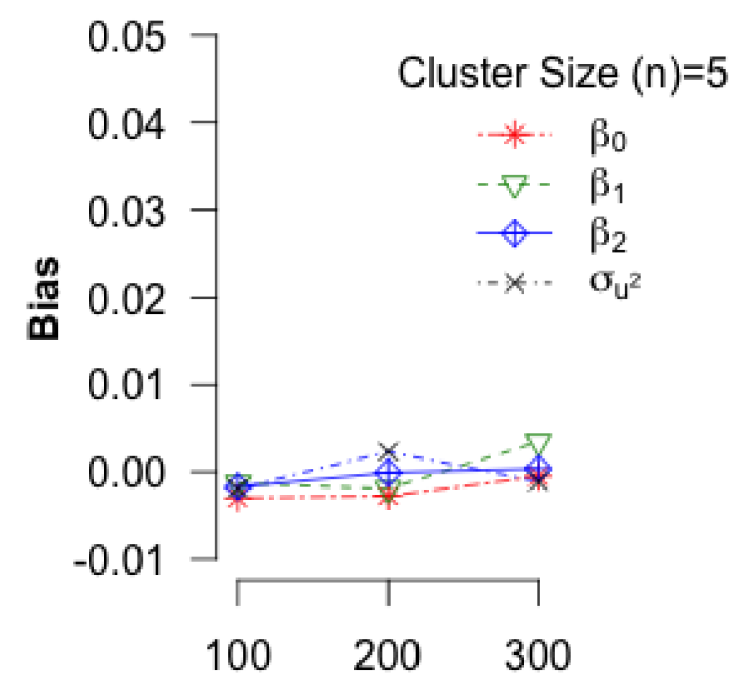

Number of Cluster $(\mathrm{N})$

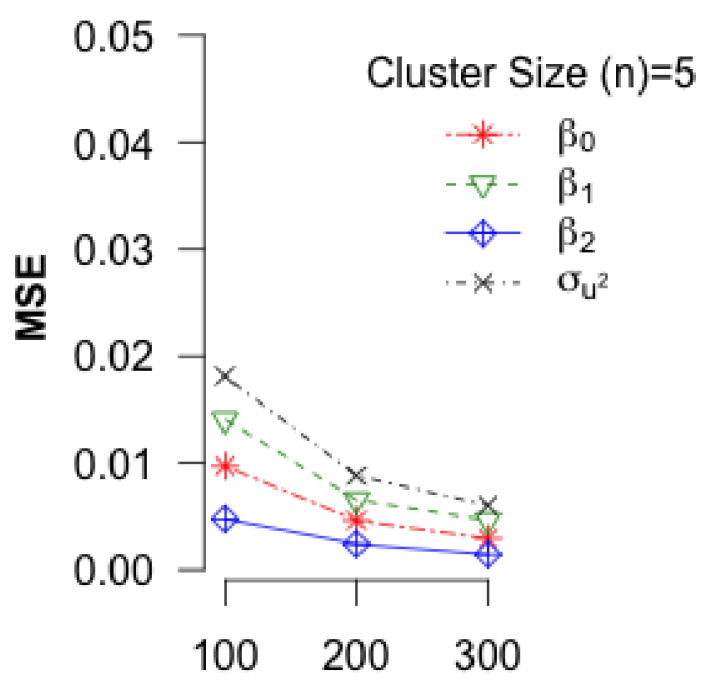

Number of Cluster $(\mathbf{N})$

Figure 5.1: Empirical biases and mean squared errors (MSEs) of the estimators of both the regression parameters $\left(\beta_{0}, \beta_{1}, \beta_{2}\right)$ and the frailty variance component $\left(\sigma_{u}^{2}\right)$ under the correct model with frailty, when the random effects are correctly specified: $u_{i} \sim N\left(0, \sigma_{u}^{2}\right)$ where $\sigma_{u}^{2}$ $=0.5$ and the cluster size $(n)=5$. Regression parameters and variance component are fixed at $\left(\beta_{0}, \beta_{1}, \beta_{2}, \sigma_{u}^{2}\right)=(-2.53,0.5,-0.25,0.5)$. 

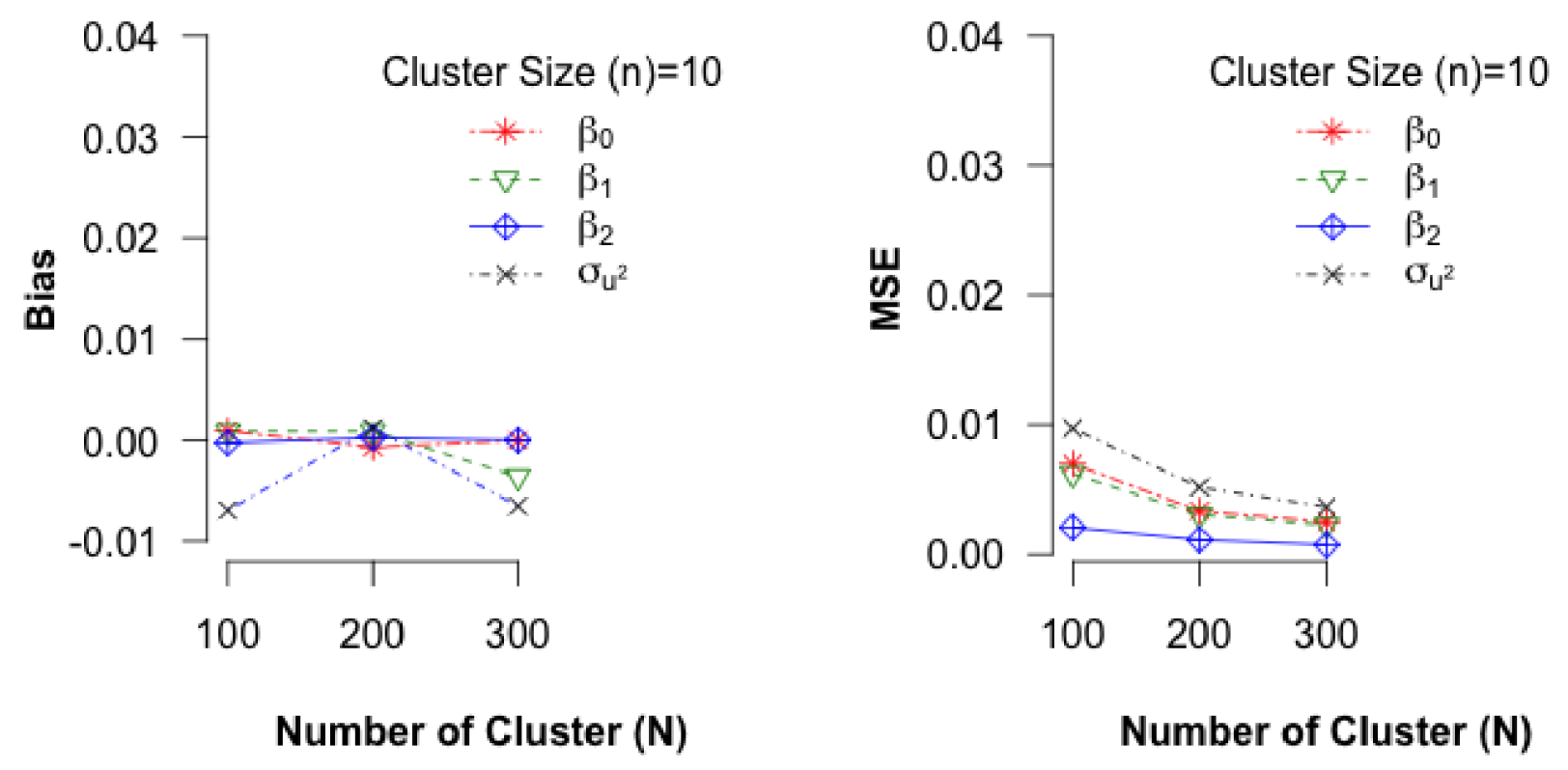

Figure 5.2: Empirical biases and mean squared errors (MSEs) of the estimators of both the regression parameters $\left(\beta_{0}, \beta_{1}, \beta_{2}\right)$ and the frailty variance component $\left(\sigma_{u}^{2}\right)$ under the correct model with frailty, when the random effects are correctly specified: $u_{i} \sim N\left(0, \sigma_{u}^{2}\right)$ where $\sigma_{u}^{2}$ $=0.5$ and the cluster size $(n)=10$. Regression parameters and variance component are fixed at $\left(\beta_{0}, \beta_{1}, \beta_{2}, \sigma_{u}^{2}\right)=(-2.53,0.5,-0.25,0.5)$. 
Table 5.7: Empirical biases and mean squared errors $(M S E s)$ of the estimators of both the regression parameters $\left(\beta_{0}, \beta_{1}, \beta_{2}\right)$ and the frailty variance component $\left(\sigma_{u}^{2}\right)$ under the correct model with frailty, when the random effects are correctly specified: $u_{i} \sim$ $N\left(0, \sigma_{u}^{2}\right)$ where $\sigma_{u}^{2}=1$. Regression parameters and variance component are fixed at $\left(\beta_{0}, \beta_{1}, \beta_{2}, \sigma_{u}^{2}\right)=(-2.53,0.5,-0.25,0.5)$.

\begin{tabular}{|c|c|c|c|c|c|c|c|c|c|c|}
\hline \multirow[b]{2}{*}{$\sigma_{u}^{2}$} & \multirow{2}{*}{$\begin{array}{c}\text { number of } \\
\text { clusters }\end{array}$} & \multirow{2}{*}{$\begin{array}{c}\text { cluster } \\
\text { size }\end{array}$} & \multicolumn{4}{|c|}{ Bias } & \multicolumn{4}{|c|}{ MSE } \\
\hline & & & $\beta_{0}$ & $\beta_{1}$ & $\beta_{2}$ & $\sigma_{u}^{2}$ & $\beta_{0}$ & $\beta_{1}$ & $\beta_{2}$ & $\sigma_{u}^{2}$ \\
\hline \multirow[t]{12}{*}{1} & 100 & 2 & -0.00401 & 0.00747 & 0.00344 & -0.00685 & 0.02215 & 0.04562 & 0.01412 & 0.11695 \\
\hline & & 5 & -0.00069 & 0.00707 & 0.00060 & 0.00140 & 0.01463 & 0.01532 & 0.00467 & 0.04757 \\
\hline & & 10 & 0.00775 & 0.00264 & 0.00171 & -0.01452 & 0.01229 & 0.00625 & 0.00235 & 0.03227 \\
\hline & & 40 & 0.00216 & 0.00034 & -0.00012 & -0.00171 & 0.01069 & 0.00148 & 0.00050 & 0.02404 \\
\hline & 200 & 2 & -0.00166 & 0.00118 & -0.00619 & 0.00969 & 0.01099 & 0.02214 & 0.00713 & 0.05613 \\
\hline & & 5 & -0.00174 & 0.00160 & -0.00143 & -0.00143 & 0.00763 & 0.00739 & 0.00263 & 0.02388 \\
\hline & & 10 & -0.00259 & 0.00188 & -0.00060 & -0.00423 & 0.00595 & 0.00326 & 0.00101 & 0.01642 \\
\hline & & 40 & 0.00205 & 0.00190 & -0.00046 & -0.01291 & 0.00504 & 0.00070 & 0.00025 & 0.01090 \\
\hline & 300 & 2 & -0.00408 & -0.00355 & 0.00011 & -0.00371 & 0.00801 & 0.01565 & 0.00494 & 0.03842 \\
\hline & & 5 & 0.00122 & 0.00104 & -0.00116 & 0.00247 & 0.00488 & 0.00461 & 0.00163 & 0.01496 \\
\hline & & 10 & -0.00192 & 0.00272 & -0.00063 & -0.00288 & 0.00425 & 0.00211 & 0.00072 & 0.01014 \\
\hline & & 40 & 0.00177 & -0.00037 & -0.00040 & -0.00766 & 0.00343 & 0.00049 & 0.00016 & 0.00755 \\
\hline
\end{tabular}




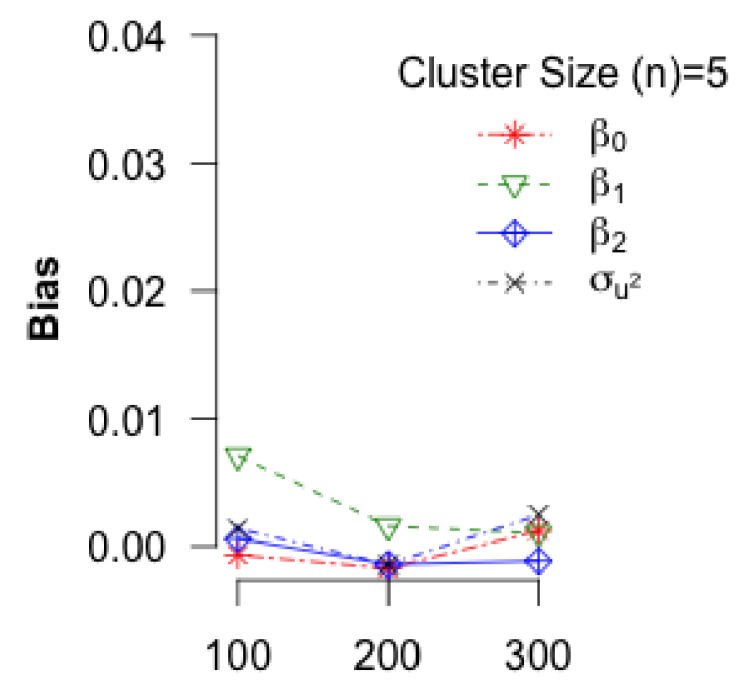

Number of Cluster $(\mathrm{N})$

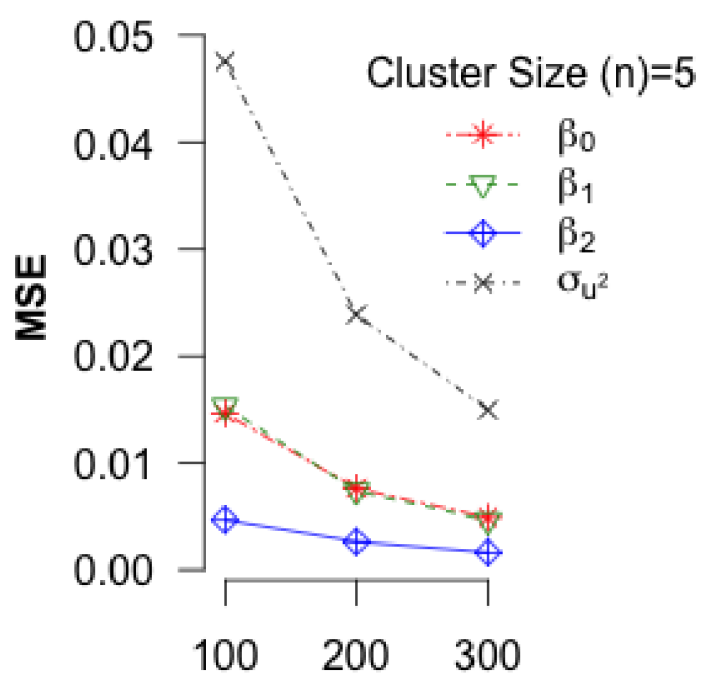

Number of Cluster (N)

Figure 5.3: Empirical biases and mean squared errors (MSEs) of the estimators of both the regression parameters $\left(\beta_{0}, \beta_{1}, \beta_{2}\right)$ and the frailty variance component $\left(\sigma_{u}^{2}\right)$ under the correct model with frailty, when the random effects are correctly specified: $u_{i} \sim N\left(0, \sigma_{u}^{2}\right)$ where $\sigma_{u}^{2}$ $=1$ and the cluster size $(n)=5$. Regression parameters and variance component are fixed at $\left(\beta_{0}, \beta_{1}, \beta_{2}, \sigma_{u}^{2}\right)=(-2.53,0.5,-0.25,0.5)$. 

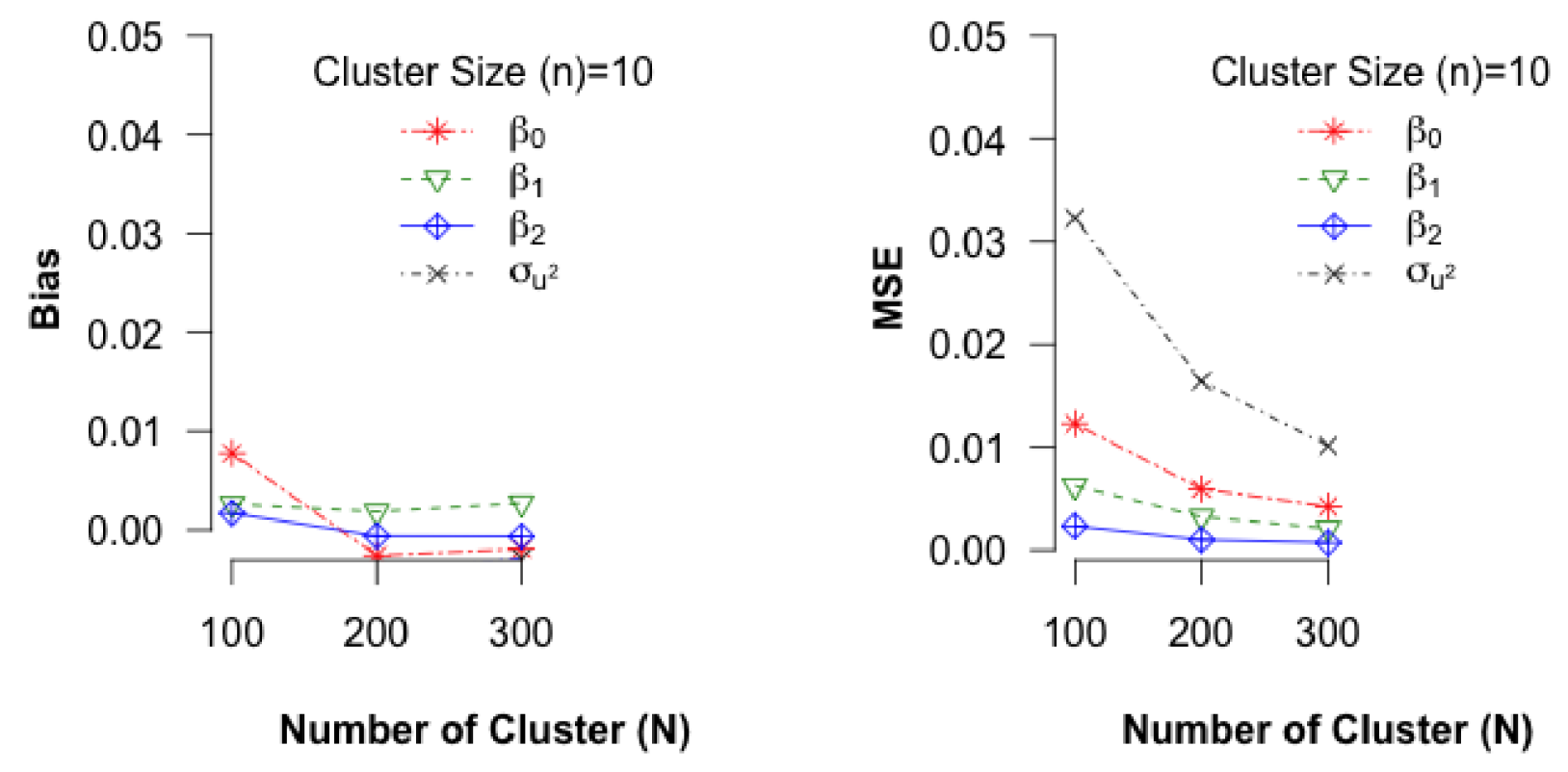

Figure 5.4: Empirical biases and mean squared errors (MSEs) of the estimators of both the regression parameters $\left(\beta_{0}, \beta_{1}, \beta_{2}\right)$ and the frailty variance component $\left(\sigma_{u}^{2}\right)$ under the correct model with frailty, when the random effects are correctly specified: $u_{i} \sim N\left(0, \sigma_{u}^{2}\right)$ where $\sigma_{u}^{2}$ $=1$ and the cluster size $(n)=10$. Regression parameters and variance component are fixed at $\left(\beta_{0}, \beta_{1}, \beta_{2}, \sigma_{u}^{2}\right)=(-2.53,0.5,-0.25,0.5)$. 
Table 5.8: Empirical biases and mean squared errors $(M S E s)$ of the estimators of both the regression parameters $\left(\beta_{0}, \beta_{1}, \beta_{2}\right)$ and the frailty variance component $\left(\sigma_{u}^{2}\right)$ under the correct model with frailty, when the random effects are correctly specified: $u_{i} \sim$ $N\left(0, \sigma_{u}^{2}\right)$ where $\sigma_{u}^{2}=4$. Regression parameters and variance component are fixed at $\left(\beta_{0}, \beta_{1}, \beta_{2}, \sigma_{u}^{2}\right)=(-2.53,0.5,-0.25,0.5)$.

\begin{tabular}{|c|c|c|c|c|c|c|c|c|c|c|}
\hline \multirow[b]{2}{*}{$\sigma_{u}^{2}$} & \multirow{2}{*}{$\begin{array}{c}\text { number of } \\
\text { clusters }\end{array}$} & \multirow{2}{*}{$\begin{array}{c}\text { cluster } \\
\text { size }\end{array}$} & \multicolumn{4}{|c|}{ Bias } & \multicolumn{4}{|c|}{ MSE } \\
\hline & & & $\beta_{0}$ & $\beta_{1}$ & $\beta_{2}$ & $\sigma_{u}^{2}$ & $\beta_{0}$ & $\beta_{1}$ & $\beta_{2}$ & $\sigma_{u}^{2}$ \\
\hline \multirow[t]{12}{*}{4} & 100 & 2 & -0.00836 & 0.00273 & -0.00391 & -0.01041 & 0.06135 & 0.06372 & 0.02239 & 0.83558 \\
\hline & & 5 & -0.00542 & 0.00106 & -0.00290 & -0.04218 & 0.04823 & 0.01742 & 0.00544 & 0.56566 \\
\hline & & 10 & -0.01399 & -0.00283 & 0.00303 & 0.00271 & 0.04042 & 0.00651 & 0.00233 & 0.44482 \\
\hline & & 40 & 0.00211 & -0.00145 & -0.00018 & -0.03913 & 0.04115 & 0.00153 & 0.00051 & 0.35237 \\
\hline & 200 & 2 & -0.00549 & 0.00698 & -0.00174 & -0.00311 & 0.02969 & 0.03560 & 0.01083 & 0.44195 \\
\hline & & 5 & 0.00532 & 0.00512 & 0.00184 & -0.00032 & 0.02197 & 0.00758 & 0.00281 & 0.26263 \\
\hline & & 10 & -0.00060 & -0.00064 & 0.00010 & -0.00851 & 0.02284 & 0.00314 & 0.00102 & 0.24349 \\
\hline & & 40 & 0.00604 & 0.00045 & -0.00048 & 0.00566 & 0.01976 & 0.00076 & 0.00024 & 0.18406 \\
\hline & 300 & 2 & -0.00073 & -0.00114 & 0.00227 & -0.02076 & 0.01946 & 0.02207 & 0.00646 & 0.29651 \\
\hline & & 5 & -0.00657 & 0.00382 & -0.00047 & 0.00493 & 0.01638 & 0.00524 & 0.00180 & 0.16884 \\
\hline & & 10 & 0.00112 & -0.00171 & -0.00019 & -0.00811 & 0.01496 & 0.00222 & 0.00074 & 0.14256 \\
\hline & & 40 & -0.00513 & -0.00094 & -0.00056 & -0.01081 & 0.01340 & 0.00048 & 0.00017 & 0.12274 \\
\hline
\end{tabular}




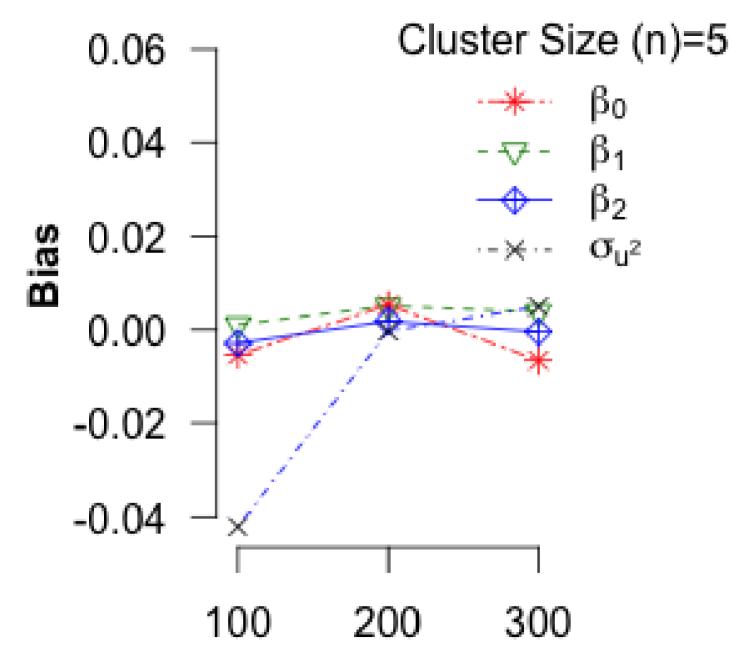

Number of Cluster (N)

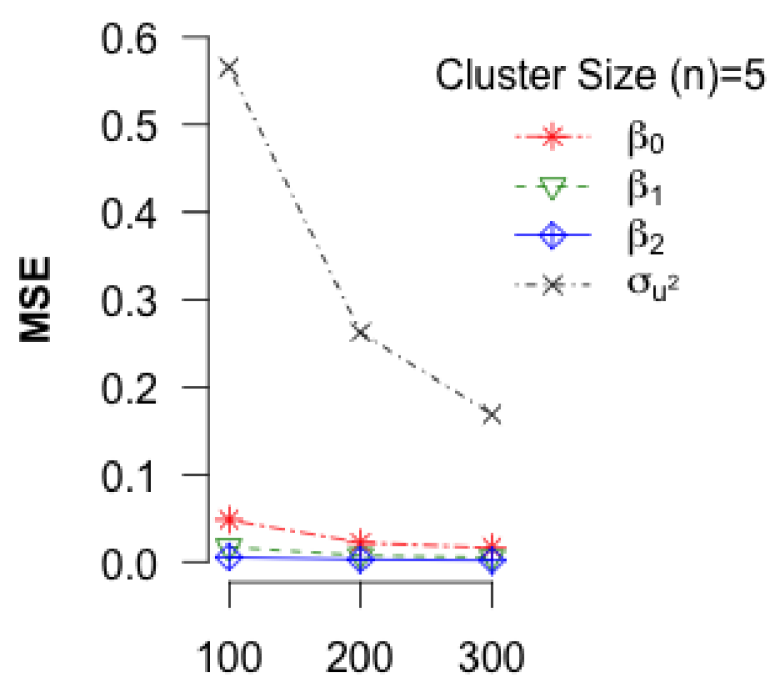

Number of Cluster (N)

Figure 5.5: Empirical biases and mean squared errors (MSEs) of the estimators of both the regression parameters $\left(\beta_{0}, \beta_{1}, \beta_{2}\right)$ and the frailty variance component $\left(\sigma_{u}^{2}\right)$ under the correct model with frailty, when the random effects are correctly specified: $u_{i} \sim N\left(0, \sigma_{u}^{2}\right)$ where $\sigma_{u}^{2}=4$ and the cluster size $(n)=5$. Regression parameters and variance component are fixed at $\left(\beta_{0}, \beta_{1}, \beta_{2}, \sigma_{u}^{2}\right)=(-2.53,0.5,-0.25,0.5)$.

\subsubsection{True Model: Frailty with $u_{i}=\sigma_{u}\left(\frac{a_{i}-\lambda}{\sqrt{\lambda}}\right)$ and $a_{i} \sim G a(\lambda, 1)$}

\subsubsection{Fitted Model: No Frailty}

Tables 5.9 - 5.16 present empirical biases and mean squared errors of the estimators of model parameters under a misspecified model without frailty, when the true frailty $u_{i}=\sigma_{u}\left(\frac{a_{i}-\lambda}{\sqrt{\lambda}}\right)$, where $a_{i} \sim G a(\lambda, 1)$ with $\lambda=(0.5,1,2,4)$.

It is clear from the tables that under misspecified models with no frailty the estimators of the regression parameters provide symmetric biases even under large samples. Also, both biases and MSEs increase when $\sigma_{u}^{2}$ increases. In addition, the scale parameter $\lambda$ influences both biases and MSEs of the estimators, which are typically increased by large values of $\lambda$. 
Table 5.9: Empirical biases and mean squared errors (MSEs) of the estimators of the regression parameters $\left(\beta_{0}, \beta_{1}, \beta_{2}\right)$ under misspecified model with no frailty, when the true model is frailty with $u_{i}=\sigma_{u}\left(\frac{a_{i}-\lambda}{\sqrt{\lambda}}\right)$ and $a_{i} \sim G a(\lambda, 1)$ for $\lambda=0.5, \quad \sigma_{u}^{2}=0,0.05$. Regression parameters are fixed at $\left(\beta_{0}, \beta_{1}, \beta_{2}\right)=(-2.53,0.5,-0.25)$.

\begin{tabular}{|c|c|c|c|c|c|c|c|c|c|}
\hline \multirow[b]{2}{*}{$\lambda$} & \multirow[b]{2}{*}{$\sigma_{u}^{2}$} & \multirow{2}{*}{$\begin{array}{c}\text { number of } \\
\text { clusters }\end{array}$} & \multirow{2}{*}{$\begin{array}{c}\text { cluster } \\
\text { size }\end{array}$} & \multicolumn{3}{|c|}{ Bias } & \multicolumn{3}{|c|}{ MSE } \\
\hline & & & & $\beta_{0}$ & $\beta_{1}$ & $\beta_{2}$ & $\beta_{0}$ & $\beta_{1}$ & $\beta_{2}$ \\
\hline \multirow[t]{12}{*}{0.5} & 0 & 100 & 2 & 0.00203 & 0.00393 & -0.00609 & 0.00926 & 0.02940 & 0.01002 \\
\hline & & & 5 & -0.00123 & -0.00220 & -0.00098 & 0.00367 & 0.01000 & 0.00397 \\
\hline & & & 10 & 0.00109 & -0.00072 & -0.00100 & 0.00182 & 0.00551 & 0.00186 \\
\hline & & & 40 & -0.00036 & -0.00104 & -0.00113 & 0.00048 & 0.00142 & 0.00046 \\
\hline & & 200 & 2 & 0.00307 & -0.00174 & 0.00369 & 0.00426 & 0.01258 & 0.00460 \\
\hline & & & 5 & 0.00158 & 0.00184 & 0.00066 & 0.00181 & 0.00550 & 0.00169 \\
\hline & & & 10 & 0.00161 & 0.00072 & -0.00271 & 0.00086 & 0.00268 & 0.00087 \\
\hline & & & 40 & -0.00042 & -0.00041 & 0.00024 & 0.00022 & 0.00064 & 0.00020 \\
\hline & & 300 & 2 & -0.00226 & -0.00299 & -0.00100 & 0.00298 & 0.00891 & 0.00307 \\
\hline & & & 5 & 0.00215 & -0.00080 & 0.00122 & 0.00122 & 0.00362 & 0.00126 \\
\hline & & & 10 & -0.00026 & 0.00010 & -0.00047 & 0.00056 & 0.00181 & 0.00062 \\
\hline & & & 40 & 0.00037 & -0.00029 & 0.00009 & 0.00015 & 0.00045 & 0.00015 \\
\hline \multirow[t]{12}{*}{0.5} & 0.05 & 100 & 2 & -0.00765 & 0.00097 & 0.00172 & 0.00925 & 0.02802 & 0.01081 \\
\hline & & & 5 & -0.00604 & -0.00699 & 0.00181 & 0.00420 & 0.01127 & 0.00359 \\
\hline & & & 10 & -0.00521 & -0.00171 & 0.00291 & 0.00218 & 0.00530 & 0.00196 \\
\hline & & & 40 & -0.00515 & -0.00471 & 0.00383 & 0.00080 & 0.00136 & 0.00049 \\
\hline & & 200 & 2 & -0.00289 & -0.00241 & -0.00060 & 0.00529 & 0.01425 & 0.00454 \\
\hline & & & 5 & -0.00523 & -0.00356 & 0.00153 & 0.00195 & 0.00525 & 0.00171 \\
\hline & & & 10 & -0.00575 & -0.00537 & 0.00047 & 0.00114 & 0.00287 & 0.00093 \\
\hline & & & 40 & -0.00416 & -0.00598 & 0.00241 & 0.00044 & 0.00072 & 0.00022 \\
\hline & & 300 & 2 & -0.00170 & 0.00103 & 0.00611 & 0.00319 & 0.00962 & 0.00305 \\
\hline & & & 5 & -0.00481 & -0.00567 & 0.00428 & 0.00145 & 0.00369 & 0.00126 \\
\hline & & & 10 & -0.00533 & -0.00513 & 0.00238 & 0.00076 & 0.00182 & 0.00061 \\
\hline & & & 40 & -0.00523 & -0.00412 & 0.00260 & 0.00034 & 0.00050 & 0.00016 \\
\hline
\end{tabular}


Table 5.10: Empirical biases and mean squared errors (MSEs) of the estimators of the regression parameters $\left(\beta_{0}, \beta_{1}, \beta_{2}\right)$ under misspecified model with no frailty, when the true model is frailty with $u_{i}=\sigma_{u}\left(\frac{a_{i}-\lambda}{\sqrt{\lambda}}\right)$ and $a_{i} \sim G a(\lambda, 1)$ for $\lambda=0.5, \quad \sigma_{u}^{2}=0.1,0.2$. Regression parameters are fixed $\mathrm{a}\left(\beta_{0}, \beta_{1}, \beta_{2}\right)=(-2.53,0.5,-0.25)$.

\begin{tabular}{|c|c|c|c|c|c|c|c|c|c|}
\hline \multirow[b]{2}{*}{$\lambda$} & \multirow[b]{2}{*}{$\sigma_{u}^{2}$} & \multirow{2}{*}{$\begin{array}{c}\text { number of } \\
\text { clusters }\end{array}$} & \multirow{2}{*}{$\begin{array}{c}\text { cluster } \\
\text { size } \\
\end{array}$} & \multicolumn{3}{|c|}{ Bias } & \multicolumn{3}{|c|}{ MSE } \\
\hline & & & & $\beta_{0}$ & $\beta_{1}$ & $\beta_{2}$ & $\beta_{0}$ & $\beta_{1}$ & $\beta_{2}$ \\
\hline \multirow[t]{12}{*}{0.5} & 0.1 & 100 & 2 & -0.00893 & -0.00372 & 0.00169 & 0.01045 & 0.02958 & 0.01042 \\
\hline & & & 5 & -0.00954 & -0.01248 & 0.00291 & 0.00463 & 0.01246 & 0.00365 \\
\hline & & & 10 & -0.00961 & -0.00675 & 0.00479 & 0.00266 & 0.00547 & 0.00190 \\
\hline & & & 40 & -0.01063 & -0.00807 & 0.00471 & 0.00130 & 0.00155 & 0.00050 \\
\hline & & 200 & 2 & -0.01049 & -0.00889 & 0.00085 & 0.00485 & 0.01498 & 0.00489 \\
\hline & & & 5 & -0.01023 & -0.00986 & 0.00208 & 0.00246 & 0.00589 & 0.00199 \\
\hline & & & 10 & -0.01254 & -0.01233 & 0.00466 & 0.00138 & 0.00310 & 0.00102 \\
\hline & & & 40 & -0.01092 & -0.00770 & 0.00475 & 0.00072 & 0.00073 & 0.00026 \\
\hline & & 300 & 2 & -0.00839 & -0.00818 & 0.00489 & 0.00388 & 0.01051 & 0.00305 \\
\hline & & & 5 & -0.01111 & -0.00744 & 0.00407 & 0.00150 & 0.00390 & 0.00117 \\
\hline & & & 10 & -0.01240 & -0.00764 & 0.00426 & 0.00105 & 0.00185 & 0.00067 \\
\hline & & & 40 & -0.01025 & -0.00935 & 0.00451 & 0.00052 & 0.00057 & 0.00018 \\
\hline \multirow[t]{12}{*}{0.5} & 2.0 & 100 & 2 & -0.02479 & -0.01453 & 0.00605 & 0.01176 & 0.02938 & 0.01075 \\
\hline & & & 5 & -0.01974 & -0.01499 & 0.00963 & 0.00569 & 0.01183 & 0.00402 \\
\hline & & & 10 & -0.02403 & -0.01199 & 0.00618 & 0.00369 & 0.00618 & 0.00202 \\
\hline & & & 40 & -0.02460 & -0.01707 & 0.00781 & 0.00246 & 0.00173 & 0.00054 \\
\hline & & 200 & 2 & -0.02355 & -0.01358 & 0.00714 & 0.00584 & 0.01595 & 0.00501 \\
\hline & & & 5 & -0.02247 & -0.01915 & 0.00663 & 0.00328 & 0.00615 & 0.00183 \\
\hline & & & 10 & -0.02292 & -0.02024 & 0.00772 & 0.00216 & 0.00342 & 0.00099 \\
\hline & & & 40 & -0.02305 & -0.01565 & 0.00769 & 0.00142 & 0.00096 & 0.00029 \\
\hline & & 300 & 2 & -0.02307 & -0.01525 & 0.00729 & 0.00406 & 0.00981 & 0.00348 \\
\hline & & & 5 & -0.02292 & -0.01038 & 0.00731 & 0.00220 & 0.00403 & 0.00143 \\
\hline & & & 10 & -0.02333 & -0.01619 & 0.00604 & 0.00159 & 0.00216 & 0.00064 \\
\hline & & & 40 & -0.02246 & -0.01600 & 0.00776 & 0.00111 & 0.00075 & 0.00021 \\
\hline
\end{tabular}


Table 5.11: Empirical biases and mean squared errors (MSEs) of the estimators of the regression parameters $\left(\beta_{0}, \beta_{1}, \beta_{2}\right)$ under misspecified model with no frailty, when the true model is frailty with $u_{i}=\sigma_{u}\left(\frac{a_{i}-\lambda}{\sqrt{\lambda}}\right)$ and $a_{i} \sim G a(\lambda, 1)$ for $\lambda=1, \quad \sigma_{u}^{2}=0,0.05$. Regression parameters are fixed at $\left(\beta_{0}, \beta_{1}, \beta_{2}\right)=(-2.53,0.5,-0.25)$.

\begin{tabular}{|c|c|c|c|c|c|c|c|c|c|}
\hline \multirow[b]{2}{*}{$\lambda$} & \multirow[b]{2}{*}{$\sigma_{u}^{2}$} & \multirow{2}{*}{$\begin{array}{c}\text { number of } \\
\text { clusters }\end{array}$} & \multirow{2}{*}{$\begin{array}{l}\text { cluster } \\
\text { size } \\
\end{array}$} & \multicolumn{3}{|c|}{ Bias } & \multicolumn{3}{|c|}{ MSE } \\
\hline & & & & $\beta_{0}$ & $\beta_{1}$ & $\beta_{2}$ & $\beta_{0}$ & $\beta_{1}$ & $\beta_{2}$ \\
\hline \multirow[t]{11}{*}{1} & 0 & 100 & 2 & 0.00744 & 0.00530 & 0.00361 & 0.00865 & 0.02932 & 0.00918 \\
\hline & & & 5 & 0.00230 & 0.00422 & -0.00161 & 0.00356 & 0.00988 & 0.00336 \\
\hline & & & 10 & 0.00238 & -0.00072 & -0.00221 & 0.00192 & 0.00528 & 0.00173 \\
\hline & & & 40 & 0.00100 & -0.00022 & 0.00023 & 0.00046 & 0.00129 & 0.00048 \\
\hline & & 200 & 2 & 0.00049 & -0.00354 & -0.00017 & 0.00433 & 0.01198 & 0.00474 \\
\hline & & & 5 & -0.00023 & 0.00001 & -0.00104 & 0.00186 & 0.00587 & 0.00188 \\
\hline & & & 10 & 0.00018 & 0.00073 & -0.00044 & 0.00089 & 0.00277 & 0.00092 \\
\hline & & & 40 & -0.00019 & -0.00015 & 0.00040 & 0.00023 & 0.00072 & 0.00023 \\
\hline & & 300 & 2 & 0.00242 & 0.00084 & 0.00263 & 0.00317 & 0.00905 & 0.00299 \\
\hline & & & 5 & -0.00117 & 0.00256 & -0.00182 & 0.00115 & 0.00333 & 0.00116 \\
\hline & & & 10 & 0.00002 & 0.00050 & -0.00011 & 0.00058 & 0.00180 & 0.00059 \\
\hline & & & 40 & 0.000003 & 0.00081 & 0.00014 & 0.00014 & 0.00044 & 0.00015 \\
\hline \multirow[t]{12}{*}{1} & 0.05 & 100 & 2 & 0.00210 & -0.01023 & 0.00025 & 0.00885 & 0.02829 & 0.00953 \\
\hline & & & 5 & -0.00567 & 0.00181 & 0.00086 & 0.00408 & 0.01124 & 0.00390 \\
\hline & & & 10 & -0.00366 & -0.00176 & 0.00232 & 0.00219 & 0.00572 & 0.00187 \\
\hline & & & 40 & -0.00453 & -0.00668 & 0.00239 & 0.00090 & 0.00150 & 0.00047 \\
\hline & & 200 & 2 & -0.00450 & -0.00142 & -0.00256 & 0.00478 & 0.01478 & 0.00446 \\
\hline & & & 5 & -0.00259 & -0.00665 & 0.00152 & 0.00208 & 0.00591 & 0.00178 \\
\hline & & & 10 & -0.00616 & -0.00587 & 0.00140 & 0.00128 & 0.00255 & 0.00095 \\
\hline & & & 40 & -0.00334 & -0.00612 & 0.00348 & 0.00046 & 0.00069 & 0.00025 \\
\hline & & 300 & 2 & -0.00511 & 0.00032 & 0.00292 & 0.00308 & 0.00914 & 0.00316 \\
\hline & & & 5 & -0.00613 & -0.00384 & 0.00153 & 0.00140 & 0.00341 & 0.00126 \\
\hline & & & 10 & -0.00527 & -0.00347 & 0.00213 & 0.00075 & 0.00174 & 0.00062 \\
\hline & & & 40 & -0.00408 & -0.00452 & 0.00147 & 0.00031 & 0.00046 & 0.00016 \\
\hline
\end{tabular}




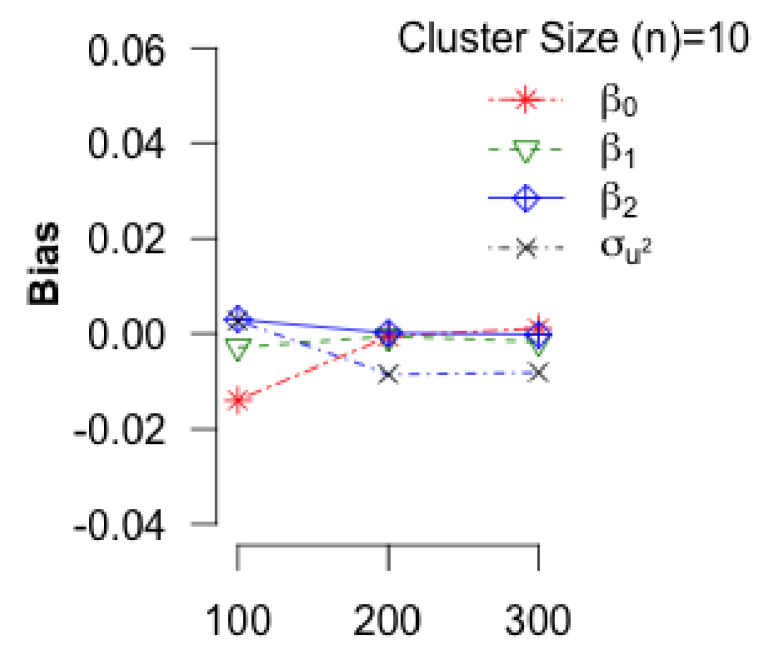

Number of Cluster (N)

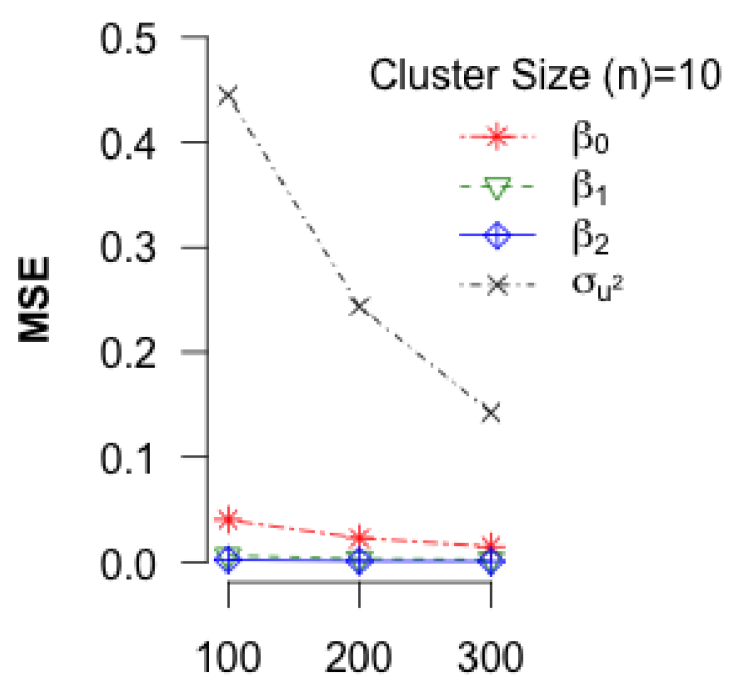

Number of Cluster (N)

Figure 5.6: Empirical biases and mean squared errors (MSEs) of the estimators of both the regression parameters $\left(\beta_{0}, \beta_{1}, \beta_{2}\right)$ and the frailty variance component $\left(\sigma_{u}^{2}\right)$ under the correct model with frailty, when the random effects are correctly specified: $u_{i} \sim N\left(0, \sigma_{u}^{2}\right)$ where $\sigma_{u}^{2}=4$ and the cluster size $(n)=10$. Regression parameters and variance component are fixed at $\left(\beta_{0}, \beta_{1}, \beta_{2}, \sigma_{u}^{2}\right)=(-2.53,0.5,-0.25,0.5)$.

\subsubsection{Fitted Model: Frailty with $u_{i} \sim N\left(0, \sigma_{u}^{2}\right)$}

Tables $5.17-5.19$ present empirical biases and MSEs of the estimators of the regression parameters $\left(\beta_{0}, \beta_{1}, \beta_{2}\right)$ and the frailty variance component $\left(\sigma_{u}^{2}\right)$ under misspecified frailty distribution, $u_{i}=\sigma_{u}\left(\frac{a_{i}-\lambda}{\sqrt{\lambda}}\right)$ where $a_{i} \sim G a(\lambda, 1)$.

It is obvious from the tables that both biases and MSEs of the model parameters tend to increase with increased values of $\sigma_{u}^{2}$. However, for fixed $\sigma_{u}^{2}$, the biases and MSEs decrease with increased values of the scale parameter $\lambda$. 
Table 5.12: Empirical biases and mean squared errors (MSEs) of the estimators of the regression parameters $\left(\beta_{0}, \beta_{1}, \beta_{2}\right)$ under misspecified model with no frailty, when the true model is frailty with $u_{i}=\sigma_{u}\left(\frac{a_{i}-\lambda}{\sqrt{\lambda}}\right)$ and $a_{i} \sim G a(\lambda, 1)$ for $\lambda=1, \quad \sigma_{u}^{2}=0.1,0.2$. Regression parameters are fixed at $\left(\beta_{0}, \beta_{1}, \beta_{2}\right)=(-2.53,0.5,-0.25)$.

\begin{tabular}{|c|c|c|c|c|c|c|c|c|c|}
\hline \multirow[b]{2}{*}{$\lambda$} & \multirow[b]{2}{*}{$\sigma_{u}^{2}$} & \multirow{2}{*}{$\begin{array}{c}\text { number of } \\
\text { clusters }\end{array}$} & \multirow{2}{*}{$\begin{array}{c}\text { cluster } \\
\text { size } \\
\end{array}$} & \multicolumn{3}{|c|}{ Bias } & \multicolumn{3}{|c|}{ MSE } \\
\hline & & & & $\beta_{0}$ & $\beta_{1}$ & $\beta_{2}$ & $\beta_{0}$ & $\beta_{1}$ & $\beta_{2}$ \\
\hline \multirow[t]{12}{*}{1} & 0.1 & 100 & 2 & -0.00963 & -0.01441 & 0.00226 & 0.00972 & 0.02813 & 0.01031 \\
\hline & & & 5 & -0.01020 & -0.00982 & 0.00574 & 0.00467 & 0.01109 & 0.00410 \\
\hline & & & 10 & -0.00885 & -0.01203 & 0.00249 & 0.00274 & 0.00559 & 0.00190 \\
\hline & & & 40 & -0.01194 & -0.01018 & 0.00510 & 0.00141 & 0.00150 & 0.00053 \\
\hline & & 200 & 2 & -0.00405 & -0.00708 & 0.00361 & 0.00466 & 0.01462 & 0.00496 \\
\hline & & & 5 & -0.01012 & -0.00945 & 0.00417 & 0.00235 & 0.00590 & 0.00198 \\
\hline & & & 10 & -0.00877 & -0.01332 & 0.00412 & 0.00143 & 0.00313 & 0.00103 \\
\hline & & & 40 & -0.01117 & -0.01020 & 0.00479 & 0.00072 & 0.00083 & 0.00026 \\
\hline & & 300 & 2 & -0.01185 & -0.00520 & 0.00285 & 0.00343 & 0.00971 & 0.00342 \\
\hline & & & 5 & -0.01204 & -0.01042 & 0.00327 & 0.00171 & 0.00379 & 0.00136 \\
\hline & & & 10 & -0.01006 & -0.01144 & 0.00399 & 0.00103 & 0.00210 & 0.00065 \\
\hline & & & 40 & -0.00887 & -0.01025 & 0.00457 & 0.00051 & 0.00056 & 0.00017 \\
\hline \multirow[t]{12}{*}{1} & 0.2 & 100 & 2 & -0.02070 & -0.00740 & 0.00039 & 0.01215 & 0.03157 & 0.00965 \\
\hline & & & 5 & -0.01902 & -0.01574 & 0.00699 & 0.00560 & 0.01181 & 0.00399 \\
\hline & & & 10 & -0.02301 & -0.02157 & 0.00759 & 0.00405 & 0.00656 & 0.00220 \\
\hline & & & 40 & -0.02269 & -0.01613 & 0.00806 & 0.00247 & 0.00174 & 0.00057 \\
\hline & & 200 & 2 & -0.02166 & -0.01878 & 0.00367 & 0.00551 & 0.01506 & 0.00525 \\
\hline & & & 5 & -0.01941 & -0.01985 & 0.00852 & 0.00291 & 0.00636 & 0.00214 \\
\hline & & & 10 & -0.02281 & -0.01699 & 0.00935 & 0.00212 & 0.00333 & 0.00108 \\
\hline & & & 40 & -0.02023 & -0.01850 & 0.00965 & 0.00134 & 0.00109 & 0.00034 \\
\hline & & 300 & 2 & -0.02059 & -0.02199 & 0.00740 & 0.00417 & 0.01061 & 0.00341 \\
\hline & & & 5 & -0.02068 & -0.02040 & 0.00935 & 0.00215 & 0.00434 & 0.00139 \\
\hline & & & 10 & -0.02234 & -0.01568 & 0.00931 & 0.00169 & 0.00214 & 0.00070 \\
\hline & & & 40 & -0.02150 & -0.01821 & 0.00941 & 0.00107 & 0.00082 & 0.00026 \\
\hline
\end{tabular}


Table 5.13: Empirical biases and mean squared errors (MSEs) of the estimators of the regression parameters $\left(\beta_{0}, \beta_{1}, \beta_{2}\right)$ under misspecified model with no frailty, when the true model is frailty with $u_{i}=\sigma_{u}\left(\frac{a_{i}-\lambda}{\sqrt{\lambda}}\right)$ and $a_{i} \sim G a(\lambda, 1)$ for $\lambda=2, \sigma_{u}^{2}=0,0.05$. Regression parameters are fixed at $\left(\beta_{0}, \beta_{1}, \beta_{2}\right)=(-2.53,0.5,-0.25)$.

\begin{tabular}{|c|c|c|c|c|c|c|c|c|c|}
\hline \multirow[b]{2}{*}{$\lambda$} & \multirow[b]{2}{*}{$\sigma_{u}^{2}$} & \multirow{2}{*}{$\begin{array}{c}\text { number of } \\
\text { clusters }\end{array}$} & \multirow{2}{*}{$\begin{array}{c}\text { cluster } \\
\text { size } \\
\end{array}$} & \multicolumn{3}{|c|}{ Bias } & \multicolumn{3}{|c|}{ MSE } \\
\hline & & & & $\beta_{0}$ & $\beta_{1}$ & $\beta_{2}$ & $\beta_{0}$ & $\beta_{1}$ & $\beta_{2}$ \\
\hline \multirow[t]{12}{*}{2} & 0 & 100 & 2 & 0.00004 & 0.00235 & -0.00013 & 0.00895 & 0.02762 & 0.00960 \\
\hline & & & 5 & 0.00346 & -0.00079 & 0.00088 & 0.00370 & 0.01039 & 0.00355 \\
\hline & & & 10 & -0.00010 & 0.00574 & -0.00114 & 0.00181 & 0.00566 & 0.00182 \\
\hline & & & 40 & 0.00046 & 0.00017 & -0.00041 & 0.00047 & 0.00132 & 0.00044 \\
\hline & & 200 & 2 & 0.00461 & 0.00202 & -0.00284 & 0.00445 & 0.01424 & 0.00431 \\
\hline & & & 5 & -0.00071 & 0.00163 & 0.00011 & 0.00190 & 0.00575 & 0.00179 \\
\hline & & & 10 & 0.00094 & -0.00208 & -0.00112 & 0.00097 & 0.00281 & 0.00090 \\
\hline & & & 40 & 0.00032 & -0.00088 & -0.00017 & 0.00024 & 0.00070 & 0.00023 \\
\hline & & 300 & 2 & 0.00031 & 0.00532 & -0.00274 & 0.00301 & 0.00906 & 0.00279 \\
\hline & & & 5 & 0.00282 & -0.00156 & 0.00178 & 0.00120 & 0.00341 & 0.00117 \\
\hline & & & 10 & -0.00068 & 0.00064 & -0.00012 & 0.00061 & 0.00187 & 0.00056 \\
\hline & & & 40 & 0.00010 & 0.00034 & -0.00062 & 0.00015 & 0.00044 & 0.00015 \\
\hline \multirow[t]{12}{*}{2} & 0.05 & 100 & 2 & 0.00256 & -0.00218 & 0.00045 & 0.01039 & 0.02937 & 0.00963 \\
\hline & & & 5 & -0.00202 & -0.00890 & 0.00082 & 0.00410 & 0.01055 & 0.00377 \\
\hline & & & 10 & -0.00593 & -0.00155 & 0.00131 & 0.00245 & 0.00588 & 0.00194 \\
\hline & & & 40 & -0.00414 & -0.00463 & 0.00184 & 0.00093 & 0.00146 & 0.00050 \\
\hline & & 200 & 2 & -0.00252 & -0.00502 & 0.00169 & 0.00458 & 0.01407 & 0.00503 \\
\hline & & & 5 & -0.00483 & -0.00142 & 0.00147 & 0.00221 & 0.00562 & 0.00162 \\
\hline & & & 10 & -0.00354 & -0.00513 & 0.00345 & 0.00120 & 0.00299 & 0.00099 \\
\hline & & & 40 & -0.00677 & -0.00484 & 0.00188 & 0.00049 & 0.00078 & 0.00023 \\
\hline & & 300 & 2 & -0.00580 & -0.00159 & 0.00114 & 0.00342 & 0.00873 & 0.00288 \\
\hline & & & 5 & -0.00299 & -0.00625 & 0.00326 & 0.00149 & 0.00355 & 0.00120 \\
\hline & & & 10 & -0.00364 & -0.00489 & 0.00349 & 0.00078 & 0.00182 & 0.00064 \\
\hline & & & 40 & -0.00329 & -0.00556 & 0.00202 & 0.00031 & 0.00050 & 0.00015 \\
\hline
\end{tabular}


Table 5.14: Empirical biases and mean squared errors (MSEs) of the estimators of the regression parameters $\left(\beta_{0}, \beta_{1}, \beta_{2}\right)$ under misspecified model with no frailty, when the true model is frailty with $u_{i}=\sigma_{u}\left(\frac{a_{i}-\lambda}{\sqrt{\lambda}}\right)$ and $a_{i} \sim G a(\lambda, 1)$ for $\lambda=2, \sigma_{u}^{2}=0.1,0.2$. Regression parameters are fixed at $\left(\beta_{0}, \beta_{1}, \beta_{2}\right)=(-2.53,0.5,-0.25)$.

\begin{tabular}{|c|c|c|c|c|c|c|c|c|c|}
\hline \multirow[b]{2}{*}{$\lambda$} & \multirow[b]{2}{*}{$\sigma_{u}^{2}$} & \multirow{2}{*}{$\begin{array}{c}\text { number of } \\
\text { clusters }\end{array}$} & \multirow{2}{*}{$\begin{array}{c}\text { cluster } \\
\text { size } \\
\end{array}$} & \multicolumn{3}{|c|}{ Bias } & \multicolumn{3}{|c|}{ MSE } \\
\hline & & & & $\beta_{0}$ & $\beta_{1}$ & $\beta_{2}$ & $\beta_{0}$ & $\beta_{1}$ & $\beta_{2}$ \\
\hline \multirow[t]{12}{*}{2} & 0.1 & 100 & 2 & -0.01483 & -0.00846 & 0.00413 & 0.01049 & 0.03220 & 0.00966 \\
\hline & & & 5 & -0.00838 & -0.01025 & 0.00200 & 0.00445 & 0.01161 & 0.00414 \\
\hline & & & 10 & -0.00785 & -0.00997 & 0.00582 & 0.00286 & 0.00596 & 0.00179 \\
\hline & & & 40 & -0.00949 & -0.00935 & 0.00638 & 0.00148 & 0.00157 & 0.00053 \\
\hline & & 200 & 2 & -0.01099 & -0.00028 & 0.00763 & 0.00546 & 0.01346 & 0.00495 \\
\hline & & & 5 & -0.00902 & -0.00938 & 0.00330 & 0.00237 & 0.00571 & 0.00197 \\
\hline & & & 10 & -0.00819 & -0.00865 & 0.00572 & 0.00150 & 0.00299 & 0.00101 \\
\hline & & & 40 & -0.00935 & -0.01089 & 0.00457 & 0.00075 & 0.00089 & 0.00025 \\
\hline & & 300 & 2 & -0.00742 & -0.00804 & 0.00280 & 0.00354 & 0.00996 & 0.00316 \\
\hline & & & 5 & -0.00969 & -0.01030 & 0.00370 & 0.00149 & 0.00353 & 0.00130 \\
\hline & & & 10 & -0.01028 & -0.00663 & 0.00416 & 0.00099 & 0.00209 & 0.00064 \\
\hline & & & 40 & -0.00941 & -0.01072 & 0.00498 & 0.00053 & 0.00059 & 0.00019 \\
\hline \multirow[t]{12}{*}{2} & 0.2 & 100 & 2 & -0.02013 & -0.01324 & 0.01042 & 0.01124 & 0.03003 & 0.01058 \\
\hline & & & 5 & -0.01750 & -0.01737 & 0.01031 & 0.00631 & 0.01230 & 0.00399 \\
\hline & & & 10 & -0.01822 & -0.02325 & 0.00935 & 0.00357 & 0.00658 & 0.00212 \\
\hline & & & 40 & -0.02012 & -0.01798 & 0.01017 & 0.00268 & 0.00174 & 0.00058 \\
\hline & & 200 & 2 & -0.01985 & -0.01930 & 0.00685 & 0.00545 & 0.01536 & 0.00545 \\
\hline & & & 5 & -0.02139 & -0.01952 & 0.01072 & 0.00319 & 0.00615 & 0.00220 \\
\hline & & & 10 & -0.02022 & -0.01629 & 0.00905 & 0.00212 & 0.00321 & 0.00108 \\
\hline & & & 40 & -0.01936 & -0.01800 & 0.00984 & 0.00144 & 0.00112 & 0.00033 \\
\hline & & 300 & 2 & -0.01599 & -0.02033 & 0.00878 & 0.00406 & 0.01066 & 0.00345 \\
\hline & & & 5 & -0.01880 & -0.01968 & 0.00768 & 0.00219 & 0.00414 & 0.00140 \\
\hline & & & 10 & -0.01959 & -0.02131 & 0.00882 & 0.00155 & 0.00240 & 0.00078 \\
\hline & & & 40 & -0.01871 & -0.01827 & 0.00985 & 0.00108 & 0.00082 & 0.00026 \\
\hline
\end{tabular}


Table 5.15: Empirical biases and mean squared errors (MSEs) of the estimators of the regression parameters $\left(\beta_{0}, \beta_{1}, \beta_{2}\right)$ under misspecified model with no frailty, when the true model is frailty with $u_{i}=\sigma_{u}\left(\frac{a_{i}-\lambda}{\sqrt{\lambda}}\right)$ and $a_{i} \sim G a(\lambda, 1)$ for $\lambda=4, \sigma_{u}^{2}=0,0.05$. Regression parameters are fixed at $\left(\beta_{0}, \beta_{1}, \beta_{2}\right)=(-2.53,0.5,-0.25)$.

\begin{tabular}{|c|c|c|c|c|c|c|c|c|c|}
\hline \multirow[b]{2}{*}{$\lambda$} & \multirow[b]{2}{*}{$\sigma_{u}^{2}$} & \multirow{2}{*}{$\begin{array}{c}\text { number of } \\
\text { clusters }\end{array}$} & \multirow{2}{*}{$\begin{array}{c}\text { cluster } \\
\text { size } \\
\end{array}$} & \multicolumn{3}{|c|}{ Bias } & \multicolumn{3}{|c|}{ MSE } \\
\hline & & & & $\beta_{0}$ & $\beta_{1}$ & $\beta_{2}$ & $\beta_{0}$ & $\beta_{1}$ & $\beta_{2}$ \\
\hline \multirow[t]{12}{*}{4} & 0 & 100 & 2 & -0.00070 & 0.00434 & -0.00297 & 0.00816 & 0.02755 & 0.00917 \\
\hline & & & 5 & 0.00421 & 0.00533 & 0.00001 & 0.00365 & 0.01126 & 0.00373 \\
\hline & & & 10 & -0.00030 & 0.00166 & 0.00255 & 0.00190 & 0.00536 & 0.00182 \\
\hline & & & 40 & 0.00040 & -0.00084 & -0.00017 & 0.00043 & 0.00132 & 0.00046 \\
\hline & & 200 & 2 & -0.00045 & 0.00238 & 0.00104 & 0.00451 & 0.01328 & 0.00419 \\
\hline & & & 5 & 0.00193 & 0.00055 & -0.00089 & 0.00173 & 0.00516 & 0.00187 \\
\hline & & & 10 & 0.00001 & 0.00248 & -0.00051 & 0.00091 & 0.00288 & 0.00088 \\
\hline & & & 40 & -0.00109 & 0.00213 & 0.00007 & 0.00022 & 0.00067 & 0.00020 \\
\hline & & 300 & 2 & 0.00324 & 0.00182 & -0.00123 & 0.00282 & 0.00911 & 0.00297 \\
\hline & & & 5 & -0.00101 & 0.00132 & 0.00031 & 0.00118 & 0.00358 & 0.00115 \\
\hline & & & 10 & 0.00053 & 0.00202 & -0.00002 & 0.00061 & 0.00186 & 0.00060 \\
\hline & & & 40 & -0.00016 & 0.00069 & 0.00011 & 0.00013 & 0.00045 & 0.00016 \\
\hline \multirow[t]{12}{*}{4} & 0.05 & 100 & 2 & 0.00142 & -0.00318 & -0.00005 & 0.01003 & 0.02797 & 0.00959 \\
\hline & & & 5 & -0.00281 & -0.00515 & 0.00186 & 0.00416 & 0.01108 & 0.00360 \\
\hline & & & 10 & -0.00128 & -0.00689 & 0.00052 & 0.00234 & 0.00544 & 0.00175 \\
\hline & & & 40 & -0.00466 & -0.00687 & 0.00217 & 0.00100 & 0.00141 & 0.00046 \\
\hline & & 200 & 2 & -0.00532 & -0.00320 & 0.00023 & 0.00470 & 0.01376 & 0.00439 \\
\hline & & & 5 & -0.00584 & -0.00331 & -0.00028 & 0.00212 & 0.00552 & 0.00178 \\
\hline & & & 10 & -0.00344 & -0.00557 & 0.00045 & 0.00108 & 0.00284 & 0.00091 \\
\hline & & & 40 & -0.00352 & -0.00530 & 0.00236 & 0.00047 & 0.00071 & 0.00024 \\
\hline & & 300 & 2 & -0.00250 & -0.00272 & 0.00404 & 0.00330 & 0.00912 & 0.00309 \\
\hline & & & 5 & -0.00446 & -0.00299 & 0.00170 & 0.00138 & 0.00364 & 0.00126 \\
\hline & & & 10 & -0.00513 & -0.00369 & 0.00127 & 0.00081 & 0.00188 & 0.00061 \\
\hline & & & 40 & -0.00387 & -0.00522 & 0.00292 & 0.00031 & 0.00046 & 0.00017 \\
\hline
\end{tabular}


Table 5.16: Empirical biases and mean squared errors (MSEs) of the estimators of the regression parameters $\left(\beta_{0}, \beta_{1}, \beta_{2}\right)$ under misspecified model with no frailty, when the true model is frailty with $u_{i}=\sigma_{u}\left(\frac{a_{i}-\lambda}{\sqrt{\lambda}}\right)$ and $a_{i} \sim G a(\lambda, 1)$ for $\lambda=4, \sigma_{u}^{2}=0.1,0.2$. Regression parameters are fixed at $\left(\beta_{0}, \beta_{1}, \beta_{2}\right)=(-2.53,0.5,-0.25)$.

\begin{tabular}{|c|c|c|c|c|c|c|c|c|c|}
\hline \multirow[b]{2}{*}{$\lambda$} & \multirow[b]{2}{*}{$\sigma_{u}^{2}$} & \multirow{2}{*}{$\begin{array}{c}\text { number of } \\
\text { clusters }\end{array}$} & \multirow{2}{*}{$\begin{array}{c}\text { cluster } \\
\text { size } \\
\end{array}$} & \multicolumn{3}{|c|}{ Bias } & \multicolumn{3}{|c|}{ MSE } \\
\hline & & & & $\beta_{0}$ & $\beta_{1}$ & $\beta_{2}$ & $\beta_{0}$ & $\beta_{1}$ & $\beta_{2}$ \\
\hline \multirow[t]{12}{*}{4} & 0.1 & 100 & 2 & -0.00485 & -0.00334 & 0.00779 & 0.01097 & 0.02887 & 0.01079 \\
\hline & & & 5 & -0.00516 & -0.01189 & 0.00160 & 0.00467 & 0.01146 & 0.00404 \\
\hline & & & 10 & -0.00723 & -0.01179 & 0.00325 & 0.00294 & 0.00549 & 0.00191 \\
\hline & & & 40 & -0.01007 & -0.01065 & 0.00505 & 0.00152 & 0.00153 & 0.00050 \\
\hline & & 200 & 2 & -0.00831 & -0.00908 & 0.00570 & 0.00509 & 0.01446 & 0.00511 \\
\hline & & & 5 & -0.00775 & -0.00843 & 0.00736 & 0.00252 & 0.00600 & 0.00201 \\
\hline & & & 10 & -0.00933 & -0.01024 & 0.00452 & 0.00153 & 0.00317 & 0.00096 \\
\hline & & & 40 & -0.00914 & -0.01162 & 0.00540 & 0.00073 & 0.00078 & 0.00026 \\
\hline & & 300 & 2 & -0.00848 & -0.00899 & 0.00361 & 0.00359 & 0.00974 & 0.00340 \\
\hline & & & 5 & -0.00689 & -0.00838 & 0.00688 & 0.00153 & 0.00373 & 0.00136 \\
\hline & & & 10 & -0.00773 & -0.00941 & 0.00626 & 0.00096 & 0.00195 & 0.00075 \\
\hline & & & 40 & -0.01001 & -0.01050 & 0.00500 & 0.00058 & 0.00057 & 0.00018 \\
\hline \multirow[t]{12}{*}{4} & 0.2 & 100 & 2 & -0.01559 & -0.02132 & 0.00423 & 0.01089 & 0.03355 & 0.01042 \\
\hline & & & 5 & -0.01836 & -0.02208 & 0.00562 & 0.00561 & 0.01269 & 0.00411 \\
\hline & & & 10 & -0.01622 & -0.01892 & 0.01082 & 0.00349 & 0.00627 & 0.00207 \\
\hline & & & 40 & -0.01683 & -0.01872 & 0.00979 & 0.00236 & 0.00183 & 0.00056 \\
\hline & & 200 & 2 & -0.01475 & -0.02718 & 0.01003 & 0.00581 & 0.01584 & 0.00519 \\
\hline & & & 5 & -0.01956 & -0.01648 & 0.00798 & 0.00285 & 0.00601 & 0.00205 \\
\hline & & & 10 & -0.01846 & -0.01991 & 0.01109 & 0.00210 & 0.00340 & 0.00110 \\
\hline & & & 40 & -0.01574 & -0.01886 & 0.01019 & 0.00132 & 0.00111 & 0.00036 \\
\hline & & 300 & 2 & -0.01681 & -0.01925 & 0.00970 & 0.00398 & 0.01077 & 0.00365 \\
\hline & & & 5 & -0.01785 & -0.01749 & 0.00886 & 0.00205 & 0.00417 & 0.00136 \\
\hline & & & 10 & -0.01770 & -0.01909 & 0.01045 & 0.00148 & 0.00238 & 0.00075 \\
\hline & & & 40 & -0.01649 & -0.02051 & 0.01007 & 0.00095 & 0.00089 & 0.00027 \\
\hline
\end{tabular}


Table 5.17: Empirical biases and mean squared errors (MSEs) of the estimators of both the regression parameters $\left(\beta_{0}, \beta_{1}, \beta_{2}\right)$ and the frailty variance component $\left(\sigma_{u}^{2}\right)$ under normal frailty model, when true $u_{i}=\sigma_{u}\left(\frac{a_{i}-\lambda}{\sqrt{\lambda}}\right)$, with $a_{i} \sim G a(\lambda, 1)$ and $\left(\lambda, \sigma_{u}^{2}\right)=(0.5,0.5)$. Regression parameters and variance component are fixed at $\left(\beta_{0}, \beta_{1}, \beta_{2}, \sigma_{u}^{2}\right)=(-2.53,0.5,-0.25,0.5)$.

\begin{tabular}{|c|c|c|c|c|c|c|c|c|c|c|c|}
\hline \multirow[b]{2}{*}{$\lambda$} & \multirow[b]{2}{*}{$\sigma_{u}^{2}$} & \multirow{2}{*}{$\begin{array}{c}\text { number of } \\
\text { clusters }\end{array}$} & \multirow{2}{*}{$\begin{array}{c}\text { cluster } \\
\text { size }\end{array}$} & \multicolumn{4}{|c|}{ Bias } & \multicolumn{4}{|c|}{ MSE } \\
\hline & & & & $\beta_{0}$ & $\beta_{1}$ & $\beta_{2}$ & $\sigma_{u}^{2}$ & $\beta_{0}$ & $\beta_{1}$ & $\beta_{2}$ & $\sigma_{u}^{2}$ \\
\hline \multirow[t]{12}{*}{0.5} & 0.5 & 100 & 2 & -0.04588 & 0.00228 & -0.00024 & -0.05992 & 0.01786 & 0.03406 & 0.01278 & 0.08779 \\
\hline & & & 5 & -0.03211 & 0.01089 & -0.00212 & 0.03249 & 0.00881 & 0.01431 & 0.00505 & 0.06069 \\
\hline & & & 10 & -0.02600 & 0.00332 & -0.00403 & 0.06377 & 0.00712 & 0.00602 & 0.00217 & 0.05351 \\
\hline & & & 40 & -0.00694 & -0.00109 & -0.00116 & 0.02467 & 0.00567 & 0.00139 & 0.00051 & 0.03708 \\
\hline & & 200 & 2 & -0.03490 & 0.00230 & -0.00217 & -0.04866 & 0.00844 & 0.01896 & 0.00698 & 0.05099 \\
\hline & & & 5 & -0.03224 & 0.00440 & -0.00226 & 0.05396 & 0.00537 & 0.00799 & 0.00223 & 0.03696 \\
\hline & & & 10 & -0.02228 & 0.00444 & -0.00243 & 0.06983 & 0.00347 & 0.00330 & 0.00111 & 0.02697 \\
\hline & & & 40 & -0.00846 & 0.00192 & 0.00083 & 0.02885 & 0.00274 & 0.00073 & 0.00025 & 0.01974 \\
\hline & & 300 & 2 & -0.03829 & 0.00626 & -0.00308 & -0.05518 & 0.00629 & 0.01292 & 0.00446 & 0.03509 \\
\hline & & & 5 & -0.03290 & 0.00812 & -0.00281 & 0.04321 & 0.00402 & 0.00484 & 0.00160 & 0.02211 \\
\hline & & & 10 & -0.02535 & 0.00235 & -0.00303 & 0.06691 & 0.00285 & 0.00209 & 0.00078 & 0.02151 \\
\hline & & & 40 & -0.00842 & 0.00186 & -0.00086 & 0.03337 & 0.00188 & 0.00050 & 0.00016 & 0.01427 \\
\hline
\end{tabular}




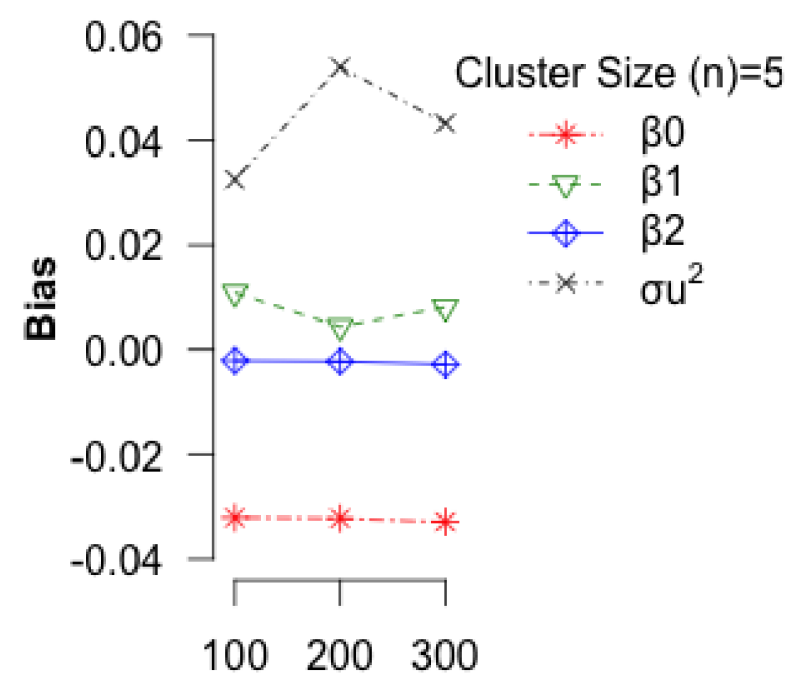

Number of Cluster (N)

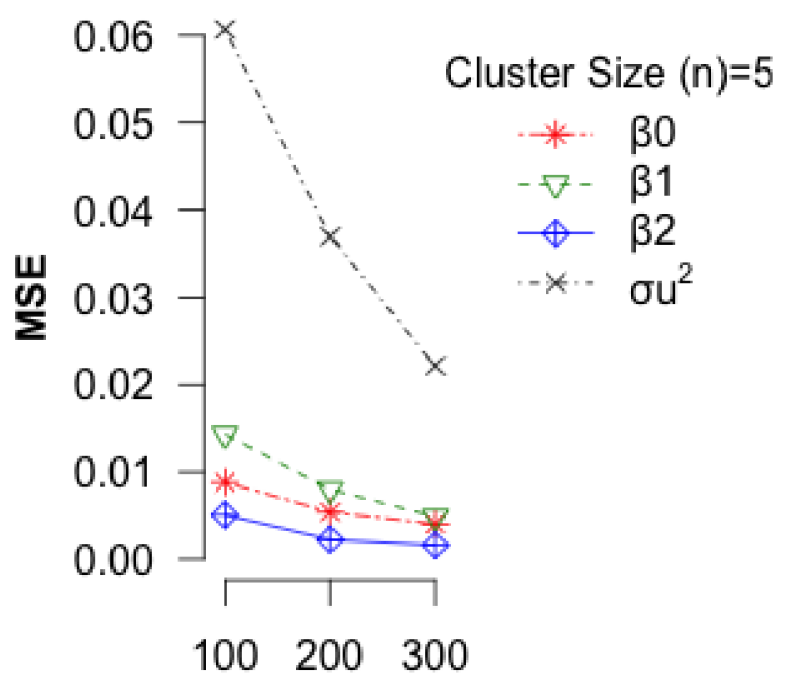

Number of Cluster $(\mathbf{N})$

Figure 5.7: Empirical biases and mean squared Errors (MSEs) of the estimators of both the regression parameters $\left(\beta_{0}, \beta_{1}, \beta_{2}\right)$ and the frailty variance component $\left(\sigma_{u}^{2}\right)$ under normal frailty model, when true $u_{i}=\sigma_{u}\left(\frac{a_{i}-\lambda}{\sqrt{\lambda}}\right)$, with $a_{i} \sim \operatorname{Ga}(\lambda, 1)$ and $\left(\lambda, \sigma_{u}^{2}\right)=(0.5,0.5)$ and the cluster size $(n)=5$. Regression parameters and variance component are fixed at $\left(\beta_{0}, \beta_{1}, \beta_{2}, \sigma_{u}^{2}\right)=(-2.53,0.5,-0.25,0.5)$. 


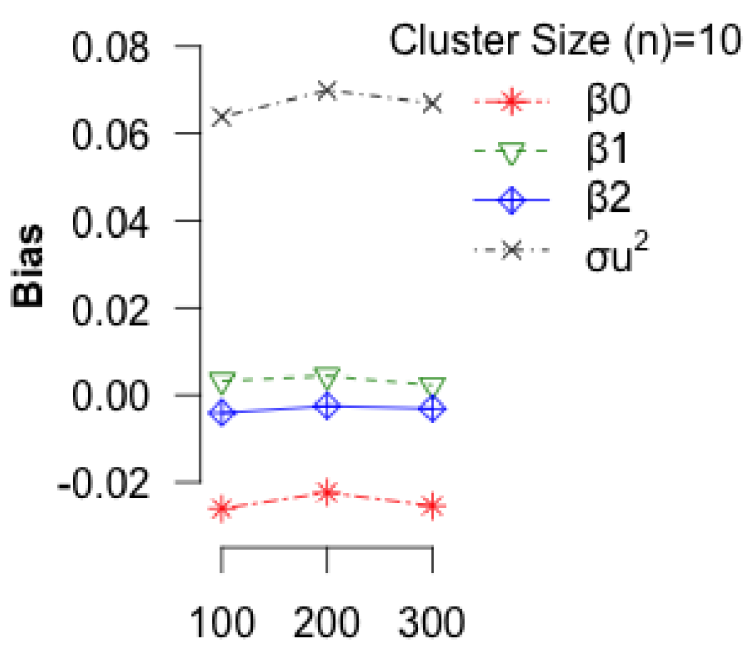

Number of Cluster (N)

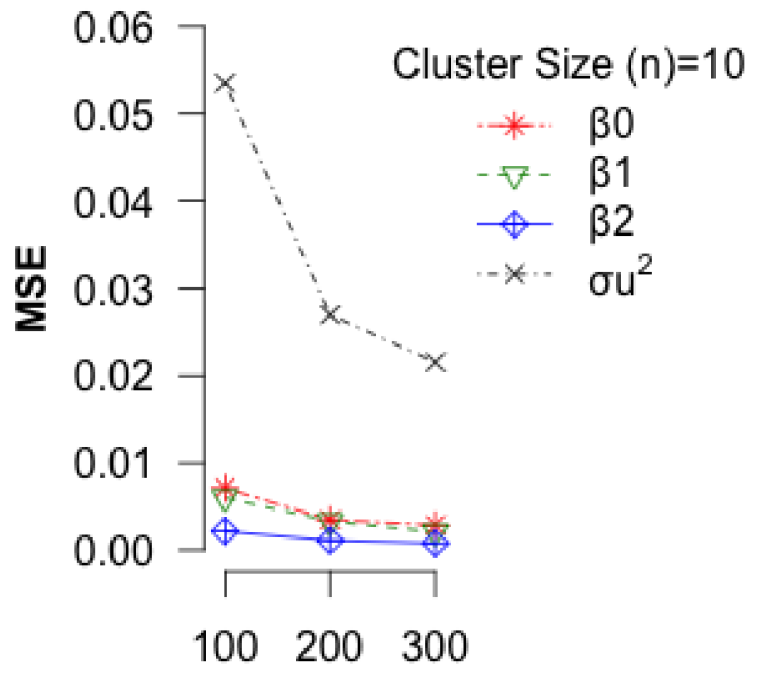

Number of Cluster (N)

Figure 5.8: Empirical biases and mean squared errors (MSEs) of the estimators of both the regression parameters $\left(\beta_{0}, \beta_{1}, \beta_{2}\right)$ and the frailty variance component $\left(\sigma_{u}^{2}\right)$ under normal frailty model, when true $u_{i}=\sigma_{u}\left(\frac{a_{i}-\lambda}{\sqrt{\lambda}}\right)$, with $a_{i} \sim G a(\lambda, 1)$ and $\left(\lambda, \sigma_{u}^{2}\right)=(0.5,0.5)$ and the cluster size $(n)=10$. Regression parameters and variance component are fixed at $\left(\beta_{0}, \beta_{1}, \beta_{2}, \sigma_{u}^{2}\right)=(-2.53,0.5,-0.25,0.5)$. 
Table 5.18: Empirical biases and mean squared errors (MSEs) of the estimators of both the regression parameters $\left(\beta_{0}, \beta_{1}, \beta_{2}\right)$ and the frailty variance component $\left(\sigma_{u}^{2}\right)$ under normal frailty model, when true $u_{i}=\sigma_{u}\left(\frac{a_{i}-\lambda}{\sqrt{\lambda}}\right)$, with $a_{i} \sim G a(\lambda, 1)$ and $\left(\lambda, \sigma_{u}^{2}\right)=(0.5,2)$. Regression parameters and variance component are fixed at $\left(\beta_{0}, \beta_{1}, \beta_{2}, \sigma_{u}^{2}\right)=(-2.53,0.5,-0.25,0.5)$.

\begin{tabular}{|c|c|c|c|c|c|c|c|c|c|c|c|}
\hline \multirow[b]{2}{*}{$\lambda$} & \multirow[b]{2}{*}{$\sigma_{u}^{2}$} & \multirow{2}{*}{$\begin{array}{c}\text { number of } \\
\text { clusters }\end{array}$} & \multirow{2}{*}{$\begin{array}{c}\text { cluster } \\
\text { size }\end{array}$} & \multicolumn{4}{|c|}{ Bias } & \multicolumn{4}{|c|}{ MSE } \\
\hline & & & & $\beta_{0}$ & $\beta_{1}$ & $\beta_{2}$ & $\sigma_{u}^{2}$ & $\beta_{0}$ & $\beta_{1}$ & $\beta_{2}$ & $\sigma_{u}^{2}$ \\
\hline \multirow[t]{12}{*}{0.5} & 2 & 100 & 2 & -0.17203 & 0.01833 & -0.01405 & 0.46516 & 0.05953 & 0.06416 & 0.02054 & 1.35025 \\
\hline & & & 5 & -0.10790 & 0.02021 & -0.00808 & 0.42840 & 0.03630 & 0.01721 & 0.00549 & 0.85244 \\
\hline & & & 10 & -0.05318 & 0.00435 & -0.00434 & 0.18206 & 0.02800 & 0.00757 & 0.00258 & 0.53646 \\
\hline & & & 40 & 0.04587 & 0.00103 & -0.00191 & 0.12124 & 0.14940 & 0.00141 & 0.00051 & 1.18009 \\
\hline & & 200 & 2 & -0.17541 & 0.03818 & -0.01258 & 0.46929 & 0.04646 & 0.03399 & 0.00940 & 0.84842 \\
\hline & & & 5 & -0.11211 & 0.01529 & -0.00793 & 0.43986 & 0.02506 & 0.00902 & 0.00278 & 0.52661 \\
\hline & & & 10 & -0.05232 & 0.00576 & -0.00379 & 0.24947 & 0.01554 & 0.00353 & 0.00120 & 0.35019 \\
\hline & & & 40 & 0.02811 & 0.00219 & -0.00108 & 0.04659 & 0.03431 & 0.00080 & 0.00025 & 0.26328 \\
\hline & & 300 & 2 & -0.18188 & 0.03005 & -0.01258 & 0.44188 & 0.04463 & 0.02331 & 0.00716 & 0.60198 \\
\hline & & & 5 & -0.11124 & 0.01408 & -0.00763 & 0.42029 & 0.02054 & 0.00607 & 0.00184 & 0.41547 \\
\hline & & & 10 & -0.05872 & 0.00911 & -0.00270 & 0.24356 & 0.01212 & 0.00248 & 0.00079 & 0.25379 \\
\hline & & & 40 & 0.03406 & 0.00075 & -0.00081 & 0.07266 & 0.02543 & 0.00055 & 0.00017 & 0.18015 \\
\hline
\end{tabular}




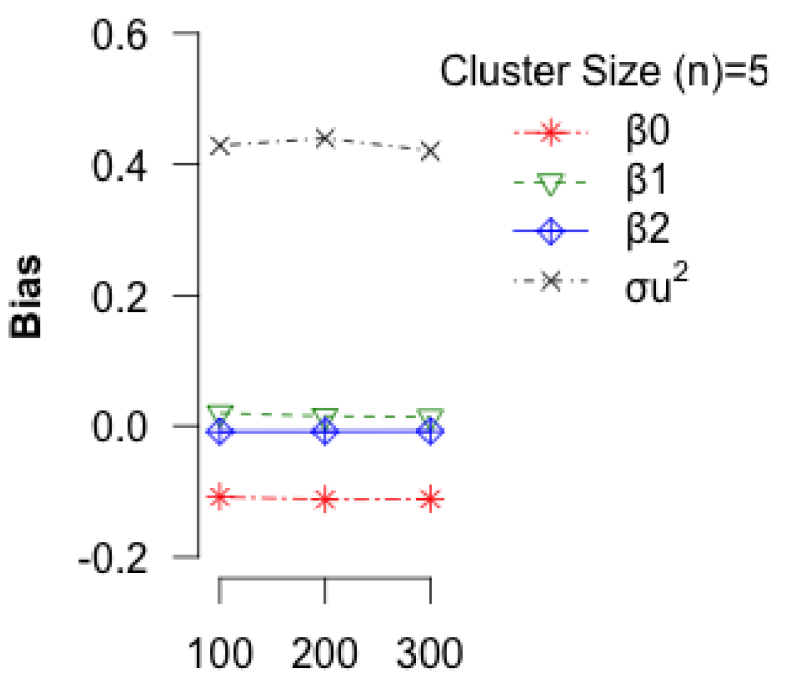

Number of Cluster (N)

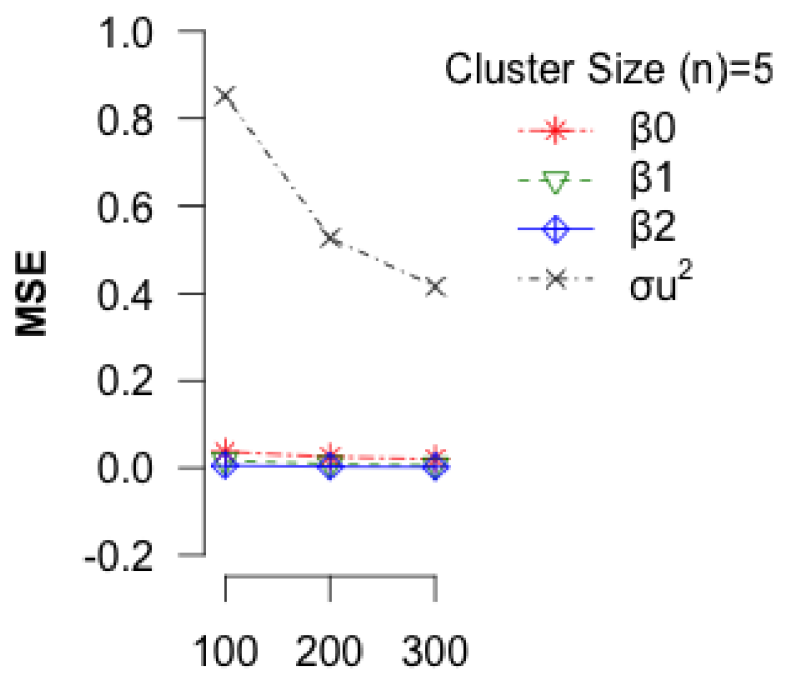

Number of Cluster (N)

Figure 5.9: Empirical biases and mean squared errors (MSEs) of the estimators of both the regression parameters $\left(\beta_{0}, \beta_{1}, \beta_{2}\right)$ and the frailty variance component $\left(\sigma_{u}^{2}\right)$ under normal frailty model, when true $u_{i}=\sigma_{u}\left(\frac{a_{i}-\lambda}{\sqrt{\lambda}}\right)$, with $a_{i} \sim G a(\lambda, 1)$ and $\left(\lambda, \sigma_{u}^{2}\right)=(0.5,2)$ and the cluster size $(n)=5$. Regression parameters and variance component are fixed at $\left(\beta_{0}, \beta_{1}, \beta_{2}, \sigma_{u}^{2}\right)=(-2.53,0.5,-0.25,0.5)$. 


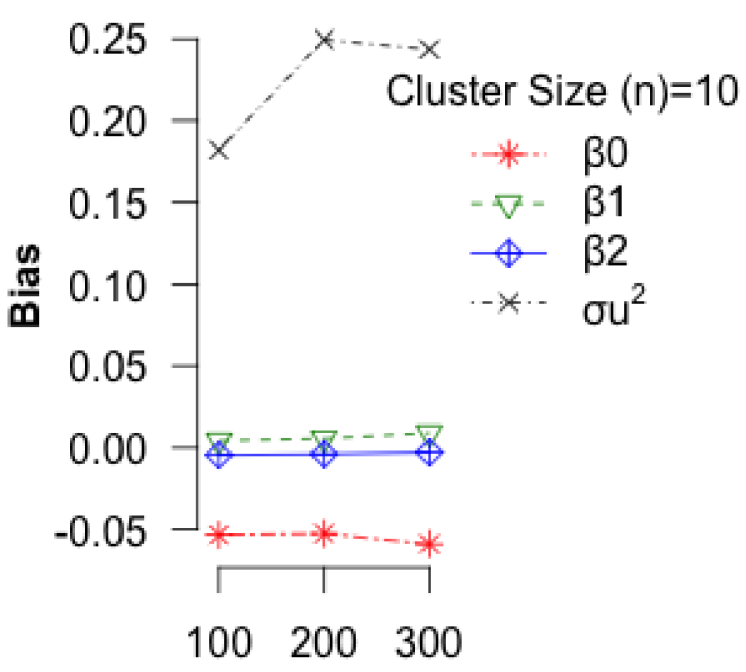

Number of Cluster (N)

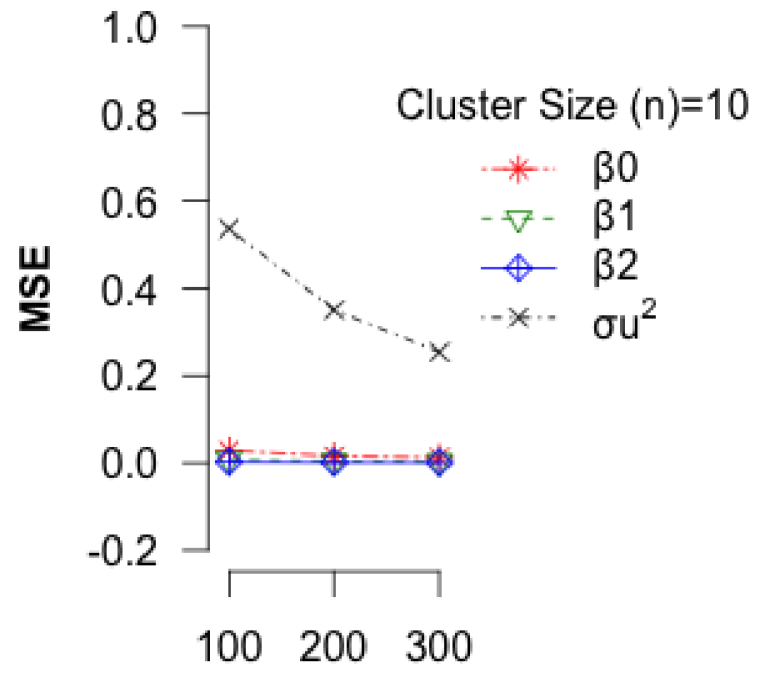

Number of Cluster (N)

Figure 5.10: Empirical biases and mean squared errors (MSEs) of the estimators of both the regression parameters $\left(\beta_{0}, \beta_{1}, \beta_{2}\right)$ and the frailty variance component $\left(\sigma_{u}^{2}\right)$ under normal frailty model, when true $u_{i}=\sigma_{u}\left(\frac{a_{i}-\lambda}{\sqrt{\lambda}}\right)$, with $a_{i} \sim G a(\lambda, 1)$ and $\left(\lambda, \sigma_{u}^{2}\right)=(0.5,2)$ and the cluster size $(n)=10$. Regression parameters and variance component are fixed at $\left(\beta_{0}, \beta_{1}, \beta_{2}, \sigma_{u}^{2}\right)=(-2.53,0.5,-0.25,0.5)$. 
Table 5.19: Empirical biases and mean squared errors (MSEs) of the estimators of both the regression parameters $\left(\beta_{0}, \beta_{1}, \beta_{2}\right)$ and the frailty variance component $\left(\sigma_{u}^{2}\right)$ under normal frailty model, when true $u_{i}=\sigma_{u}\left(\frac{a_{i}-\lambda}{\sqrt{\lambda}}\right)$, with $a_{i} \sim G a(\lambda, 1) \&$ $\left(\lambda, \sigma_{u}^{2}\right)=(2,2)$. Regression parameters and variance component are fixed at $\left(\beta_{0}, \beta_{1}, \beta_{2}, \sigma_{u}^{2}\right)=(-2.53,0.5,-0.25,0.5)$.

\begin{tabular}{|c|c|c|c|c|c|c|c|c|c|c|c|}
\hline \multirow[b]{2}{*}{$\lambda$} & \multirow[b]{2}{*}{$\sigma_{u}^{2}$} & \multirow{2}{*}{$\begin{array}{c}\text { number of } \\
\text { clusters }\end{array}$} & \multirow{2}{*}{$\begin{array}{c}\text { cluster } \\
\text { size }\end{array}$} & \multicolumn{4}{|c|}{ Bias } & \multicolumn{4}{|c|}{ MSE } \\
\hline & & & & $\beta_{0}$ & $\beta_{1}$ & $\beta_{2}$ & $\sigma_{u}^{2}$ & $\beta_{0}$ & $\beta_{1}$ & $\beta_{2}$ & $\sigma_{u}^{2}$ \\
\hline \multirow[t]{12}{*}{2} & 2 & 100 & 2 & -0.12563 & 0.01219 & -0.00669 & 0.51069 & 0.05112 & 0.05980 & 0.02160 & 0.96063 \\
\hline & & & 5 & -0.07517 & 0.00709 & -0.00617 & 0.38146 & 0.03211 & 0.01634 & 0.00566 & 0.51026 \\
\hline & & & 10 & -0.04488 & 0.00181 & -0.00161 & 0.26112 & 0.02404 & 0.00694 & 0.00237 & 0.33718 \\
\hline & & & 40 & -0.01982 & 0.00306 & 0.00068 & 0.03851 & 0.02522 & 0.00160 & 0.00052 & 0.21606 \\
\hline & & 200 & 2 & -0.12386 & 0.01762 & -0.00566 & 0.52447 & 0.03433 & 0.03079 & 0.01020 & 0.61126 \\
\hline & & & 5 & -0.07868 & 0.00688 & -0.00277 & 0.40176 & 0.01824 & 0.00765 & 0.00252 & 0.36406 \\
\hline & & & 10 & -0.04952 & 0.00469 & 0.00028 & 0.25259 & 0.01454 & 0.00344 & 0.00114 & 0.19793 \\
\hline & & & 40 & -0.00982 & 0.00082 & 0.00001 & 0.07148 & 0.01180 & 0.00074 & 0.00026 & 0.11340 \\
\hline & & 300 & 2 & -0.11873 & 0.02377 & -0.00691 & 0.51274 & 0.02661 & 0.02194 & 0.00723 & 0.48787 \\
\hline & & & 5 & -0.08060 & 0.00955 & -0.00384 & 0.38736 & 0.01501 & 0.00597 & 0.00188 & 0.27393 \\
\hline & & & 10 & -0.05438 & 0.00627 & -0.00041 & 0.23927 & 0.01110 & 0.00245 & 0.00076 & 0.14888 \\
\hline & & & 40 & -0.01146 & 0.00047 & -0.00021 & 0.07572 & 0.00745 & 0.00050 & 0.00017 & 0.07102 \\
\hline
\end{tabular}




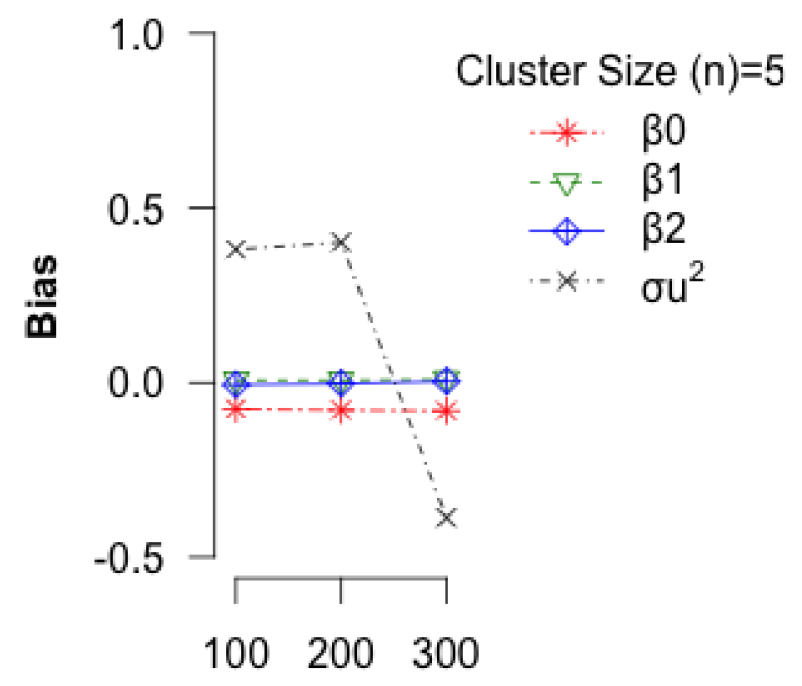

Number of Cluster (N)

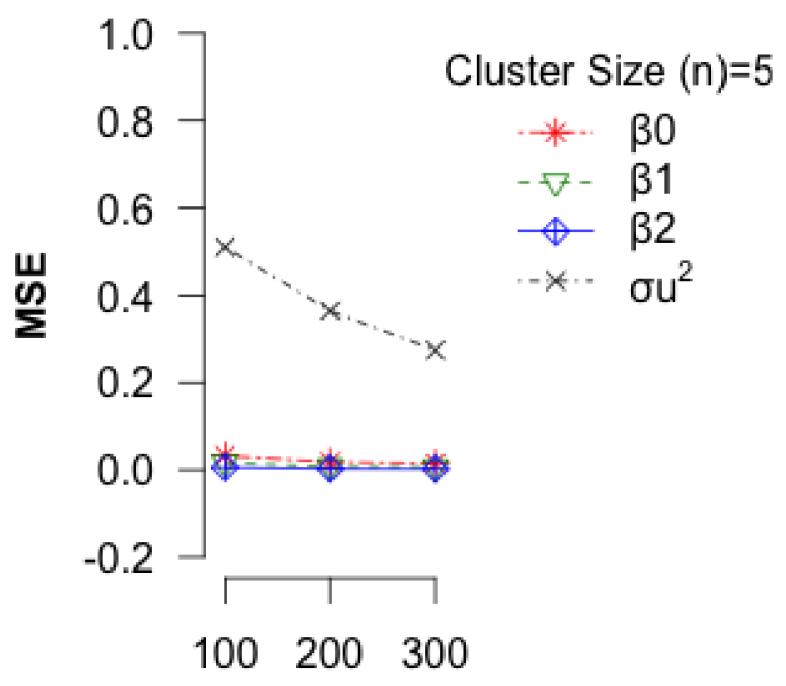

Number of Cluster (N)

Figure 5.11: Empirical biases and mean squared Errors (MSEs) of the estimators of both the regression parameters $\left(\beta_{0}, \beta_{1}, \beta_{2}\right)$ and the frailty variance component $\left(\sigma_{u}^{2}\right)$ under normal frailty model, when true $u_{i}=\sigma_{u}\left(\frac{a_{i}-\lambda}{\sqrt{\lambda}}\right)$, with $a_{i} \sim G a(\lambda, 1)$ and $\left(\lambda, \sigma_{u}^{2}\right)=(2,2)$ and the cluster size $(n)=5$. Regression parameters and variance component are fixed at $\left(\beta_{0}, \beta_{1}, \beta_{2}, \sigma_{u}^{2}\right)=(-2.53,0.5,-0.25,0.5)$. 


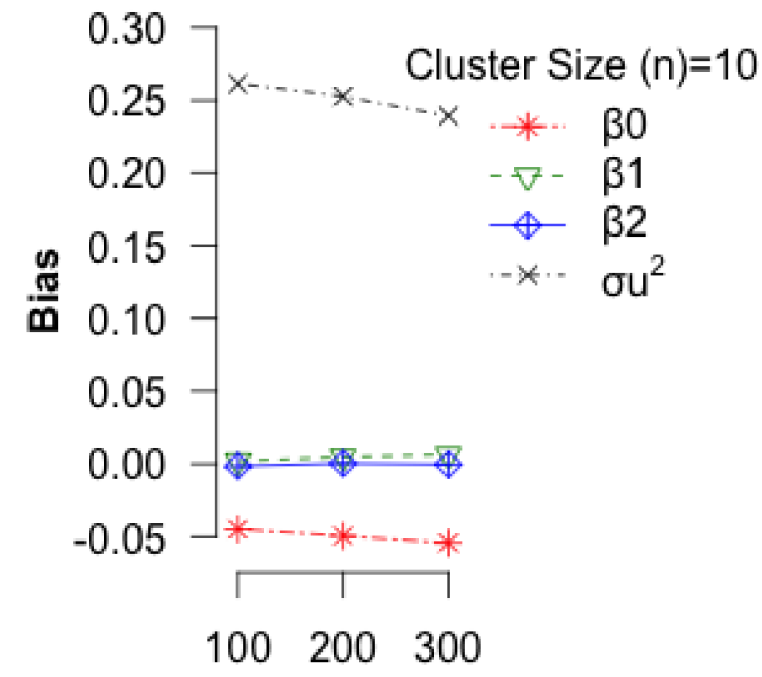

Number of Cluster (N)

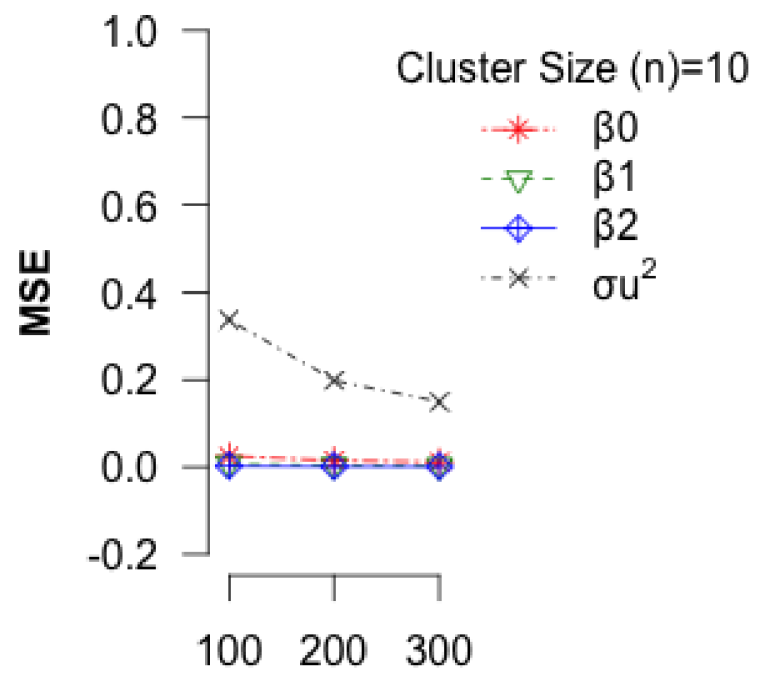

Number of Cluster (N)

Figure 5.12: Empirical biases and mean squared errors (MSEs) of the estimators of both the regression parameters $\left(\beta_{0}, \beta_{1}, \beta_{2}\right)$ and the frailty variance component $\left(\sigma_{u}^{2}\right)$ under normal frailty model, when true $u_{i}=\sigma_{u}\left(\frac{a_{i}-\lambda}{\sqrt{\lambda}}\right)$, with $a_{i} \sim G a(\lambda, 1)$ and $\left(\lambda, \sigma_{u}^{2}\right)=(2,2)$ and the cluster size $(n)=10$. Regression parameters and variance component are fixed at $\left(\beta_{0}, \beta_{1}, \beta_{2}, \sigma_{u}^{2}\right)=(-2.53,0.5,-0.25,0.5)$. 


\section{Chapter 6}

\section{Application: Bladder Cancer Data}

\subsection{Bladder Cancer Study}

In this section, we present an analysis of bladder cancer data obtained from a clinical trial conducted by the Veterans Administration Co-operative Urological Research Group (VACURG) in order to examine the hazard of numerous recurrence of tumours for patients with bladder cancer (details are given in Wei, Lin and Weissfeld [30]). This experiment was based on data collected from patients who had superficial bladder tumours before they entered the trial. At the beginning of the trial, tumours for those patients were removed transurethrally and then each patient was randomized to receive one type of three treatments (pills): placebo, pyridoxine, and thiotepa. Subsequently, any new or successive tumors found were recorded, removed each time the patients visited the clinic, and then the patients continued the treatment. According to Andrews and Herzberg [3] and Sinha [26], the term recurrence denotes the frequency of new tumours that appear in the bladder of a patient at a given visit during the study, and the time-to-recurrence of tumors were measured in month from the removal of previous tumors at a certain visit until the diagnosis of new tumours. The objective of this experiment is to investigate which type of the treatments is the most effective in reducing the recurrences of tumours in patients. In this thesis, we followed Byar [6], Wei, Lin and 
Weissfeld [30], and Sinha [26] and considered only the analysis of the first four recurrence times of tumours for patients in both placebo and thiotepa groups.

\subsubsection{The Model}

We consider modelling the first four recurrence times of tumors for 85 patients, where 47 and 38 of them were randomly assigned to take placebo and thiotepa treatments, respectively (see the actual data in Tables 7.1 and 7.2. We assume that the number of patients is the number of clusters $(i=1,2, \ldots \ldots, 85)$ and the four observations in each cluster represent recurrence times of tumour $(j=1, \ldots, 4)$. The recurrence time $T_{i j}$ for the $i^{\text {th }}$ patient were measured in months from removing the previous tumours until detecting the $j^{\text {th }}$ new tumour at a given visit. The status of those recurrence times $T_{i j}{ }^{\prime} s$ are presented by a censoring indicator $\delta_{i j}$ (which is 1 if $T_{i j}$ is observed and 0 if $T_{i j}$ is right-censored). We assume that the censoring mechanism is independent of the recurrent event process. We are interested in examining the effect of the following explanatory variables on the time-to-recurrence of tumours.

Treat $_{i}=\left(1\right.$ if the $i^{\text {th }}$ patient is from thiotepa group and 0 if the $i^{\text {th }}$ patient is from placepo group).

Number $_{i}=$ number of initial tumors for the $i^{\text {th }}$ patient ranged from 1 to 8 , where 8 denotes eight or more initial tumors.

$S i z e_{i}=$ diameter of the largest initial tumor for the $i^{\text {th }}$ patient measured in centimetres.

For the given data, we fit a proportional hazards model with a shared frailty in the form

$$
h_{i j}(t)=\exp \left(\beta_{0}+\beta_{1} \text { Treat }_{i}+\beta_{2} \text { Number }_{i}+\beta_{3} \text { Size }_{i}+u_{i}\right)
$$

for $i=1, \ldots ., 85 ; j=1, \ldots, 4$; where $t$ is time in month from the beginning of the study

until each new recurrence of tumors and $u_{i}$ is the random effect for the $i^{\text {th }}$ patient assumed to be independent and normally distributed with mean 0 and variance component $\sigma_{u}^{2}$. 
Here analyses of bladder cancer data is based on waiting times or gap times. We defined $K_{j}=T_{j}-T_{j-1}$ as the gap time between the $(j-1)^{t h}$ and $j^{t h}$ event. As patients undergo transurethral resection to remove any recent diagnostic tumors, and then protective treatment is given to slow down the occurrence of new tumors, this type of analysis based or gap times is reasonable (see actual data given in Appendix 8.2).

\subsubsection{Analysis of the Recurrent Events}

Initially, we are interested in testing the homogeneity of individuals by setting the null hypothesis that time-to-events (recurrence times of tumors) are not different between subjects for the given predictor variables $\left(H_{0}: \sigma_{u}^{2}=0\right)$, versus the alternative hypothesis that event times (recurrence times of tumors) for the same patient share an unobserved common frailty $\left(H_{a}: \sigma_{u}^{2}>0\right)$.

Table 6.1: Analysis of bladder cancer data using a standard proportional hazards model without frailty $\left(\sigma_{u}^{2}=0\right)$.

\begin{tabular}{rcccc}
\hline \hline Parameter & Estimate & SE & $\begin{array}{c}\text { Score Statistics } \\
T=\frac{U_{0}^{2}}{D_{0}}\end{array}$ & P-value \\
\hline Intercept & -2.7191 & 0.3320 & 12.0012 & 0.00027 \\
Treat & -0.5142 & 0.1992 & & \\
Number & 0.1896 & 0.0472 & & \\
Size & -0.0446 & 0.0682 & & \\
\hline \hline
\end{tabular}

For testing the hypothesis, we performed the proposed score test following Silvapulle and Silvapulle [24] and Sinha [26]. We use the score statistics $T$ given in the form (4.7). To perform the proposed test, we fit the model under the null hypothesis of no frailties ( i.e., $H_{0}: \sigma_{u}^{2}=0$ ) by using the maximum likelihood approach explained in section 4.2.2. Table 6.1 presents the value of score statistics as 12.0012 and a small p-value of 0.00027 based on the mixture of chi-square distributions $(0.5,0.5)$, which indicates clearly that there is a strong 
evidence against the null hypothesis. Thus the recurrence times of tumors between subjects for the given explanatory variables are significantly different at $5 \%$ level.

Table 6.2: Analysis of bladder cancer data using a proportional hazards model with a shared frailty $\left(\sigma_{u}^{2} \neq 0\right)$.

\begin{tabular}{cccc}
\hline \hline & & & \\
Parameters & Estimate & SE & z value \\
\hline & & & \\
Intercept & -3.0478 & 0.5624 & -5.42 \\
Treat & -0.6265 & 0.3239 & -1.93 \\
Number & 0.2485 & 0.0867 & 2.87 \\
Size & -0.0176 & 0.1086 & -0.16 \\
$\sigma_{u}^{2}$ & 0.9221 & 0.3441 & 2.68 \\
\hline \hline
\end{tabular}

Since the heterogeneity parameter $\sigma_{u}^{2}$ is significant, we fit the proportional hazards model with frailty (6.1). As mentioned before, we use the "optim" package in $\mathrm{R}$ to find maximum likelihood estimates of the regression parameters $\boldsymbol{\beta}$ and variance component $\sigma_{u}^{2}$. Table 6.2 shows the maximum likelihood estimates of the model parameters and their corresponding standard errors. In brief, it is clear that the treatment thiothepa has a positive impact on reducing the risk of recurrence of tumors, while there is an increase of this risk as the number of initial tumors increases. 


\section{Chapter 7}

\section{Conclusion}

The purpose of the thesis was to study the use of the score test for testing the significance of a frailty variance component in clustered survival data. We test the homogeneity of survival times of subjects within a cluster under the null hypothesis $H_{0}: \sigma_{u}^{2}=0$ using a shared frailty model.

The empirical level of the score test gets closer to the nominal $5 \%$ level when the number

of clusters $(N)$ and the cluster size $(n)$ increase. Also, the power of this test increases when the frailty variance component $\sigma_{u}^{2}$ increases as well as the number of clusters $(N)$ and the cluster size $(n)$ increase. We also observe similar pattern when the random effects are misspecified and obtained from a gamma distribution. The empirical level and power of the score test are not much affected by this misspecification.

For estimating the model parameters under no frailty variance component, the simulation study shows that the ML method provides estimates with smaller biases, but the biases increase as $\sigma_{u}^{2}$ under "true" frailty model increases. But under misspecified random effects, it appears that both biases and MSEs of the estimators are affected by the scale parameter $\lambda$, since they increase by the large values of $\lambda$.

When estimating under a shared frailty, the model with correctly specified random effects generally provides unbiased estimates of both the regression parameters and variance 
component. The MSEs increase as $\sigma_{u}^{2}$ increases, but they tend to decrease as the number of clusters $(N)$ and cluster size $(n)$ increase. However, for fixed $\sigma_{u}^{2}$, the biases and MSEs decrease with increased values of the scale parameter $\lambda$ in the case of misspecified random effects.

\subsection{Future Research}

As a future research, we intend to explore a parametric bootstrap method that can provide an approximate p-value of the one-sided score test for testing a frailty variance component in recurrent event data as suggested by Sinha [26]. As observed in our simulation study, the asymptotic score test based on the mixture of chi-square distributions generally provides incorrect level of significance when the sample size is small. To overcome this problem, we can adopt the parametric bootstrap method, which is a permutation score test based on randomly permuting the indices associated with the individuals of a survival model. Sinha [26] shows empirically that the bootstrap test has approximately the correct level of significance and generally more powerful than the asymptotic score test. 


\section{Chapter 8}

\section{Appendix}

\subsection{Derivation of the Score Test Statistic}

The marginal likelihood for the $i^{t h}$ individual may be expressed as

$$
\begin{aligned}
L_{i}\left(\boldsymbol{\beta}, \boldsymbol{\theta}, \sigma_{u}^{2}\right) & =E_{u_{i}}\left\{\prod_{j=1}^{n_{i}} g\left(t_{i j} \mid u_{i}\right)\right\} \\
& =E_{u_{i}}\left\{g^{*}\left(t_{i} \mid u_{i}\right)\right\} \\
& =E_{u_{i}}\left\{g^{*}\left(t_{i} \mid u_{i}=0\right)+\left.\frac{\partial g^{*}\left(t_{i} \mid u_{i}\right)}{\partial u_{i}}\right|_{u_{i}=0}\left(u_{i}-0\right)+\left.\sum_{k=2}^{\infty} \frac{\partial^{k} g^{*}\left(t_{i} \mid u_{i}\right)}{u_{i}^{k}}\right|_{u_{i}=0} \frac{\left(u_{i}-0\right)^{k}}{k !}\right\} \\
& =\left\{g^{*}\left(t_{i} \mid u_{i}=0\right)+\left.\frac{\partial g^{*}\left(t_{i} \mid u_{i}\right)}{\partial u_{i}}\right|_{u_{i}=0} E\left(u_{i}\right)+\left.\sum_{k=2}^{\infty} \frac{\partial^{k} g^{*}\left(t_{i} \mid u_{i}\right)}{u_{i}^{k}}\right|_{u_{i}=0} \frac{E\left(u_{i}\right)^{k}}{k !}\right\} \\
& =\left\{g^{*}\left(t_{i} \mid u_{i}=0\right)+\sum_{k=2}^{\infty} \frac{a_{k}}{k !} \frac{\partial^{k} g^{*}\left(t_{i} \mid u_{i}\right)}{u_{i}^{k}}\right\},
\end{aligned}
$$

where

$$
g^{*}\left(t_{i} \mid u_{i}\right)=\prod_{j=1}^{n_{i}} g\left(t_{i j} \mid u_{i}\right), E\left(u_{i}\right)=0, E\left(u_{i}^{k}\right)=a_{k} .
$$

Then the logarithm of the marginal likelihood for the $i^{\text {th }}$ individual evaluated at $\sigma_{u}^{2}=0$ is obtained as 


$$
\begin{aligned}
\left.\log L_{i}\left(\boldsymbol{\beta}, \boldsymbol{\theta}, \sigma_{u}^{2}\right)\right|_{\sigma_{u}^{2}=0} & =\log \left\{g^{*}\left(t_{i} \mid u_{i}=0\right)+\sum_{k=2}^{\infty} \frac{a_{k}}{k !} \frac{\partial^{k}}{\partial u_{i}^{k}} g^{*}\left(t_{i} \mid u_{i}\right)\right\} \\
& =\log \left\{g^{*}\left(t_{i} \mid u_{i}=0\right)\right\}
\end{aligned}
$$

where

$$
\begin{aligned}
\log \left\{g^{*}\left(t_{i} \mid u_{i}=0\right)\right\} & =\sum_{j=1}^{n_{i}} \log g\left(t_{i j} \mid u_{i}\right) \\
& =\sum_{j=1}^{n_{i}}\left\{\delta_{i j} \log h_{i j}\left(t_{i j}\right)+\log S_{i j}\left(t_{i j}\right)\right\} \\
& =\sum_{j=1}^{n_{i}}\left\{\delta_{i j}\left[\log h_{0}(t, \boldsymbol{\theta})+\boldsymbol{x}_{i j}^{\prime} \boldsymbol{\beta}+u_{i}\right]-\int_{0}^{t_{i j}} h_{i j}(i j) d t\right\} \\
& =\sum_{j=1}^{n_{i}}\left\{\delta_{i j} \log h_{0}(t, \boldsymbol{\theta})+\delta_{i j} \boldsymbol{x}_{i j}^{\prime} \boldsymbol{\beta}+\delta_{i j} u_{i}-\exp \left(\boldsymbol{x}_{i j}^{\prime} \boldsymbol{\beta}+u_{i}\right) \int_{0}^{t_{i j}} h_{0}(t, \boldsymbol{\theta}) d t\right\} \\
& =\sum_{j=1}^{n_{i}}\left\{\delta_{i j} \log h_{0}(t, \boldsymbol{\theta})+\delta_{i j} \boldsymbol{x}_{i j}^{\prime} \boldsymbol{\beta}+\delta_{i j} u_{i}-\exp \left(\boldsymbol{x}_{i j}^{\prime} \boldsymbol{\beta}+u_{i}\right) H_{0}(t, \boldsymbol{\theta})\right\} . \quad(8.2)
\end{aligned}
$$

The score function of $\sigma_{u}^{2}$ evaluated at $\sigma_{u}^{2}=0$ is obtained as

$$
U(\boldsymbol{\gamma})=\sum_{i=1}^{N} S_{i}^{*}(\boldsymbol{\gamma})
$$


where

$$
\begin{aligned}
& S_{i}^{*}(\gamma) \\
& =\left.\frac{\partial \log L_{i}\left(\boldsymbol{\beta}, \boldsymbol{\theta}, \sigma_{u}^{2}\right)}{\partial \sigma_{u}^{2}}\right|_{\sigma_{u}^{2}=0} \\
& =\left.\frac{1}{L_{i}\left(\boldsymbol{\beta}, \boldsymbol{\theta}, \sigma_{u}^{2}\right)} \frac{\partial}{\partial \sigma_{u}^{2}} L_{i}\left(\boldsymbol{\beta}, \boldsymbol{\theta}, \sigma_{u}^{2}\right)\right|_{\sigma_{u}^{2}=0} \\
& =\frac{1}{L_{i}\left(\boldsymbol{\beta}, \boldsymbol{\theta}, \sigma_{u}^{2}\right)} \frac{\partial}{\partial \sigma_{u}^{2}}\left[\left.\prod_{j=1}^{n_{i}} g\left(t_{i j} \mid u_{i}\right)\right|_{u_{i}=0}+\left.\sum_{k=2}^{\infty} \frac{a_{k}}{k !} \frac{\partial^{k}}{u_{i}^{k}} \prod_{j=1}^{n_{i}} g\left(t_{i j} \mid u_{i}\right)\right|_{u_{i}=0}\right] \\
& =\frac{1}{g^{*}\left(t_{i} \mid u_{i}\right)} \frac{\partial}{\partial \sigma_{u}^{2}}\left[\left.g^{*}\left(t_{i} \mid u_{i}\right)\right|_{u_{i}=0}+\left.\sum_{k=2}^{\infty} \frac{a_{k}}{k !} \frac{\partial^{k}}{\partial u_{i}^{k}} g^{*}\left(t_{i} \mid u_{i}\right)\right|_{u_{i}=0}\right] \\
& =\frac{1}{g^{*}\left(t_{i} \mid u_{i}\right)} \frac{\partial}{\partial \sigma_{u}^{2}}\left[\left.\sum_{k=2}^{\infty} \frac{a_{k}}{k !} \frac{\partial^{k}}{\partial u_{i}^{k}} g^{*}\left(t_{i} \mid u_{i}\right)\right|_{u_{i}=0}\right] \\
& =\frac{1}{g^{*}\left(t_{i} \mid u_{i}\right)} \frac{1}{2}\left[\frac{\partial}{\partial \sigma_{u}^{2}}\left\{\left.a_{2} \frac{\partial^{2}}{\partial u_{i}^{2}} g^{*}\left(t_{i} \mid u_{i}\right)\right|_{u_{i}=0}\right\}\right] \\
& =\frac{1}{g^{*}\left(t_{i} \mid u_{i}\right)} \frac{1}{2}\left[\frac{\partial}{\partial \sigma_{u}^{2}}\left\{\left.\sigma_{u}^{2} \frac{\partial^{2}}{\partial u_{i}^{2}} g^{*}\left(t_{i} \mid u_{i}\right)\right|_{u_{i}=0}\right\}\right] \\
& =\frac{1}{g^{*}\left(t_{i} \mid u_{i}\right)} \frac{1}{2}\left[\left.\frac{\partial^{2}}{\partial u_{i}{ }^{2}} g^{*}\left(t_{i} \mid u_{i}\right)\right|_{u_{i}=0}+0\right] \\
& =\frac{1}{g^{*}\left(t_{i} \mid u_{i}\right)} \frac{1}{2}\left[\frac{\partial}{\partial u_{i}}\left\{\left.\frac{\partial}{\partial u_{i}} g^{*}\left(t_{i} \mid u_{i}\right)\right|_{u_{i}=0}\right\}\right] \\
& =\frac{1}{g^{*}\left(t_{i} \mid u_{i}\right)} \frac{1}{2}\left[\frac{\partial}{\partial u_{i}}\left\{\left.g^{*}\left(t_{i} \mid u_{i}\right) \frac{\partial}{\partial u_{i}} \log g^{*}\left(t_{i} \mid u_{i}\right)\right|_{u_{i}=0}\right\}\right] \\
& =\frac{1}{g^{*}\left(t_{i} \mid u_{i}\right)} \frac{1}{2}\left[\left.\frac{\partial}{\partial u_{i}} g^{*}\left(t_{i} \mid u_{i}\right) \frac{\partial}{\partial u_{i}} \log g^{*}\left(t_{i} \mid u_{i}\right)\right|_{u_{i}=0}+\left.g^{*}\left(t_{i} \mid u_{i}\right) \frac{\partial^{2}}{\partial u_{i}^{2}} \log g^{*}\left(t_{i} \mid u_{i}\right)\right|_{u_{i}=0}\right] \\
& =\frac{1}{g^{*}\left(t_{i} \mid u_{i}\right)} \frac{1}{2}\left[g^{*}\left(t_{i} \mid u_{i}\right)\left\{\left.\frac{\partial}{\partial u_{i}} \log g^{*}\left(t_{i} \mid u_{i}\right)\right|_{u_{i}=0}\right\}\left\{\left.\frac{\partial}{\partial u_{i}} \log g^{*}\left(t_{i} \mid u_{i}\right)\right|_{u_{i}=0}\right\}\right. \\
& \left.+g^{*}\left(t_{i} \mid u_{i}\right)\left\{\left.\frac{\partial^{2}}{\partial u_{i}^{2}} \log g^{*}\left(t_{i} \mid u_{i}\right)\right|_{u_{i}=0}\right\}\right]
\end{aligned}
$$




$$
\begin{aligned}
& =\frac{1}{2}\left[\left\{\left.\frac{\partial}{\partial u_{i}} \log g^{*}\left(t_{i} \mid u_{i}\right)\right|_{u_{i}=0}\right\}^{2}+\left.\frac{\partial^{2}}{\partial u_{i}{ }^{2}} \log g^{*}\left(t_{i} \mid u_{i}\right)\right|_{u_{i}=0}\right] \\
& =\frac{1}{2}\left[\left\{\left.\sum_{j=1}^{n_{i}} \frac{\partial}{\partial u_{i}} \log g\left(t_{i j} \mid u_{i}\right)\right|_{u_{i}=0}\right\}^{2}+\left.\sum_{j=1}^{n_{i}} \frac{\partial^{2}}{\partial u_{i}{ }^{2}} \log g\left(t_{i j} \mid u_{i}\right)\right|_{u_{i}=0}\right] \\
& =\frac{1}{2}\left[\left\{\sum_{j=1}^{n_{i}} \delta_{i j}-\exp \left(\boldsymbol{x}_{i j}^{\prime} \boldsymbol{\beta}\right) H_{0}\left(t_{i j}\right)\right\}^{2}-\sum_{j=1}^{n_{i}} \exp \left(\boldsymbol{x}_{i j}^{\prime} \boldsymbol{\beta}\right) H_{0}\left(t_{i j}\right)\right] .
\end{aligned}
$$

Then

$$
\begin{aligned}
U(\boldsymbol{\gamma}) & =\sum_{i=1}^{N} S_{i}^{*}(\boldsymbol{\gamma}) \\
& =\sum_{i=1}^{N} \frac{1}{2}\left[\left\{\sum_{j=1}^{n_{i}} \delta_{i j}-\exp \left(\boldsymbol{x}_{i j}^{\prime} \boldsymbol{\beta}\right) H_{0}\left(t_{i j}\right)\right\}^{2}-\sum_{j=1}^{n_{i}} \exp \left(\boldsymbol{x}_{i j}^{\prime} \boldsymbol{\beta}\right) H_{0}\left(t_{i j}\right)\right]
\end{aligned}
$$

where

$$
\begin{aligned}
\left.\frac{\partial}{\partial u_{i}} \log g^{*}\left(t_{i} \mid u_{i}\right)\right|_{u_{i}=0} & =\left\{\delta_{i j}-\left.\exp \left(\boldsymbol{x}_{i j}^{\prime} \boldsymbol{\beta}+u_{i}\right) H_{0}\left(t_{i j}\right)\right|_{u_{i}=0}\right\} \\
& =\left[\delta_{i j}-\exp \left(\boldsymbol{x}_{i j}^{\prime} \boldsymbol{\beta}\right) H_{0}\left(t_{i j}\right)\right],
\end{aligned}
$$

$$
\left.\frac{\partial^{2}}{\partial u_{i}{ }^{2}} \log g^{*}\left(t_{i} \mid u_{i}\right)\right|_{u_{i}=0}=-\left.\exp \left(\boldsymbol{x}_{i j}^{\prime} \boldsymbol{\beta}+u_{i}\right) H_{0}\left(t_{i j}\right)\right|_{u_{i}=0}=-\exp \left(\boldsymbol{x}_{i j}^{\prime} \boldsymbol{\beta}\right) H_{0}\left(t_{i j}\right)
$$

The Fisher information matrix $I(\gamma)$ evaluated at $\sigma_{u}^{2}=0$ can be expressed in the form 


$$
I(\gamma)=\left(\begin{array}{cc}
I_{\gamma \gamma} & I_{\gamma \sigma} \\
I_{\sigma \gamma} & I_{\sigma \sigma}
\end{array}\right),
$$

where

$$
\begin{array}{r}
I_{\boldsymbol{\gamma} \boldsymbol{\gamma}}=-\left.\sum_{i=1}^{N} \frac{\partial^{2} \log L_{i}\left(\boldsymbol{\gamma}, \sigma_{u}^{2}\right)}{\partial \boldsymbol{\gamma} \partial \boldsymbol{\gamma}^{\prime}}\right|_{\sigma_{u}^{2}=0}, \\
I_{\boldsymbol{\gamma} \boldsymbol{\sigma}}=I_{\boldsymbol{\sigma} \gamma}^{\prime}=-\left.\sum_{i=1}^{N} \frac{\partial^{2} \log L_{i}\left(\boldsymbol{\gamma}, \sigma_{u}^{2}\right)}{\partial \boldsymbol{\gamma} \partial \sigma_{u}^{2}}\right|_{\sigma_{u}^{2}=0},
\end{array}
$$

and

$$
I_{\boldsymbol{\sigma} \boldsymbol{\sigma}}=-\left.\sum_{i=1}^{N} \frac{\partial^{2} \log L_{i}\left(\boldsymbol{\gamma}, \sigma_{u}^{2}\right)}{\partial\left(\sigma_{u}^{2}\right)^{2}}\right|_{\sigma_{u}^{2}=0}
$$

using (8.2), we have

$$
\begin{aligned}
\left.\frac{\partial \log L_{i}\left(\boldsymbol{\gamma}, \sigma_{u}^{2}\right)}{\partial \boldsymbol{\beta}}\right|_{\sigma_{u}^{2}=0} & =\frac{\partial}{\partial \boldsymbol{\beta}} \log g^{*}\left(t_{i} \mid u_{i}\right) \\
& =\sum_{j=1}^{n_{i}} \frac{\partial}{\partial \boldsymbol{\beta}} \log g\left(t_{i j} \mid u_{i}\right) \\
& =\sum_{j=1}^{n_{i}} \frac{\partial}{\partial \boldsymbol{\beta}}\left\{\delta_{i j} \log h_{0}(t, \boldsymbol{\theta})+\delta_{i j} \boldsymbol{x}_{i j}^{\prime} \boldsymbol{\beta}+\delta_{i j} u_{i}-\exp \left(\boldsymbol{x}_{i j}^{\prime} \boldsymbol{\beta}+u_{i}\right) H_{0}(t, \boldsymbol{\theta})\right\} \\
& =\sum_{j=1}^{n_{i}}\left\{\delta_{i j} \boldsymbol{x}_{i j}-\left.\boldsymbol{x}_{i j} \exp \left(\boldsymbol{x}_{i j}^{\prime} \boldsymbol{\beta}+u_{i}\right)\right|_{u_{i}=0} H_{0}\left(t_{i j}\right)\right\},
\end{aligned}
$$




$$
\begin{aligned}
\left.\frac{\partial^{2} \log L_{i}\left(\boldsymbol{\gamma}, \sigma_{u}^{2}\right)}{\partial \boldsymbol{\beta} \partial \boldsymbol{\beta}^{\prime}}\right|_{\sigma_{u}^{2}=0} & =-\left.\sum_{j=1}^{n_{i}} \boldsymbol{x}_{i j} \boldsymbol{x}_{i j}^{\prime} \exp \left(\boldsymbol{x}_{i j}^{\prime} \boldsymbol{\beta}+u_{i}\right)\right|_{u_{i}=0} H_{0}\left(t_{i j}\right) \\
& =-\sum_{j=1}^{n_{i}} \boldsymbol{x}_{i j} \boldsymbol{x}_{i j}^{\prime} \exp \left(\boldsymbol{x}_{i j}^{\prime} \boldsymbol{\beta}\right) H_{0}\left(t_{i j}\right)
\end{aligned}
$$

$$
\begin{aligned}
\left.\frac{\partial \log L_{i}\left(\boldsymbol{\gamma}, \sigma_{u}^{2}\right)}{\partial \boldsymbol{\theta}}\right|_{\sigma_{u}^{2}=0} & =\frac{\partial}{\partial \boldsymbol{\theta}} \log g^{*}\left(t_{i} \mid u_{i}\right) \\
& =\sum_{j=1}^{n_{i}} \frac{\partial}{\partial \boldsymbol{\theta}} \log g\left(t_{i j} \mid u_{i}\right) \\
& =\sum_{j=1}^{n_{i}} \frac{\partial}{\partial \boldsymbol{\theta}}\left\{\delta_{i j} \log h_{0}(t, \boldsymbol{\theta})+\delta_{i j} \boldsymbol{x}_{i j}^{\prime} \boldsymbol{\beta}+\delta_{i j} u_{i}-\exp \left(\boldsymbol{x}_{i j}^{\prime} \boldsymbol{\beta}+u_{i}\right) H_{0}(t, \boldsymbol{\theta})\right\} \\
& =\sum_{j=1}^{n_{i}}\left\{\delta_{i j} \frac{\partial \log h_{0}(t, \boldsymbol{\theta})}{\partial \boldsymbol{\theta}}-\left.\exp \left(\boldsymbol{x}_{i j}^{\prime} \boldsymbol{\beta}+u_{i}\right)\right|_{u_{i}=0} \frac{\partial H_{0}(t, \boldsymbol{\theta})}{\partial \boldsymbol{\theta}}\right\},
\end{aligned}
$$

$$
\begin{aligned}
\left.\frac{\partial \log L_{i}\left(\boldsymbol{\gamma}, \sigma_{u}^{2}\right)}{\partial \boldsymbol{\theta} \partial \boldsymbol{\beta}^{\prime}}\right|_{\sigma_{u}^{2}=0} & =-\left.\sum_{j=1}^{n_{i}} \boldsymbol{x}_{i j} \exp \left(\boldsymbol{x}_{i j}^{\prime} \boldsymbol{\beta}+u_{i}\right)\right|_{u_{i}=0} \frac{\partial H_{0}(t, \boldsymbol{\theta})}{\partial \theta} \\
& =-\sum_{j=1}^{n_{i}} \frac{\partial H_{0}(t, \boldsymbol{\theta})}{\partial \boldsymbol{\theta}} \exp \left(\boldsymbol{x}_{i j}^{\prime} \boldsymbol{\beta}\right) \boldsymbol{x}_{i j}
\end{aligned}
$$

$$
\begin{aligned}
\left.\frac{\partial^{2} \log L_{i}\left(\boldsymbol{\gamma}, \sigma_{u}^{2}\right)}{\partial \boldsymbol{\theta} \partial \boldsymbol{\theta}^{\prime}}\right|_{\sigma_{u}^{2}=0} & =\sum_{j=1}^{n_{i}}\left[\delta_{i j} \frac{\partial^{2} \log h_{0}(t, \boldsymbol{\theta})}{\partial \boldsymbol{\theta} \partial \boldsymbol{\theta}^{\prime}}-\left.\exp \left(\boldsymbol{x}_{i j}^{\prime} \boldsymbol{\beta}+u_{i}\right)\right|_{u_{i}=0} \frac{\partial^{2} H_{0}\left(t_{i j}\right)}{\partial \boldsymbol{\theta} \partial \boldsymbol{\theta}^{\prime}}\right] \\
& =\sum_{j=1}^{n_{i}}\left[\delta_{i j} \frac{\partial^{2} \log h_{0}(t, \boldsymbol{\theta})}{\partial \boldsymbol{\theta} \partial \boldsymbol{\theta}^{\prime}}-\exp \left(\boldsymbol{x}_{i j}^{\prime} \boldsymbol{\beta}\right) \frac{\partial^{2} H_{0}\left(t_{i j}\right)}{\partial \boldsymbol{\theta} \partial \boldsymbol{\theta}^{\prime}}\right]
\end{aligned}
$$




$$
\begin{aligned}
& \left.\frac{\partial \log L_{i}\left(\boldsymbol{\gamma}, \sigma_{u}^{2}\right)}{\partial \sigma_{u}^{2}}\right|_{\sigma_{u}^{2}=0}=\left.\frac{1}{2}\left[\left\{\sum_{j=1}^{n_{i}}\left[\delta_{i j}-H_{0}\left(t_{i j}\right) \exp \left(\boldsymbol{x}_{i j}^{\prime} \boldsymbol{\beta}\right)\right]\right\}^{2}-\sum_{j=1}^{n_{i}} H_{0}\left(t_{i j}\right) \exp \left(\boldsymbol{x}_{i j}^{\prime} \boldsymbol{\beta}\right)\right]\right|_{u_{i}=0}(8.12) \\
& \left.\frac{\partial \log L_{i}\left(\boldsymbol{\gamma}, \sigma_{u}^{2}\right)}{\partial \sigma_{u}^{2} \partial \boldsymbol{\beta}^{\prime}}\right|_{\sigma_{u}^{2}=0} \\
& =\frac{1}{2}\left[2 \sum_{j=1}^{n_{i}}\left\{\delta_{i j}-H_{0}\left(t_{i j}\right) \exp \left(\boldsymbol{x}_{i j}^{\prime} \boldsymbol{\beta}\right)\right\}-\left\{\sum_{j=1}^{n_{i}} H_{0}\left(t_{i j}\right) \boldsymbol{x}_{i j} \exp \left(\boldsymbol{x}_{i j}^{\prime} \boldsymbol{\beta}\right)\right\}-\sum_{j=1}^{n_{i}} H_{0}\left(t_{i j}\right) \boldsymbol{x}_{i j} \exp \left(\boldsymbol{x}_{i j}^{\prime} \boldsymbol{\beta}\right)\right] \\
& =\sum_{j=1}^{n_{i}}\left\{\delta_{i j}-H_{0}\left(t_{i j}\right) \exp \left(\boldsymbol{x}_{i j}^{\prime} \boldsymbol{\beta}\right)\right\}-\frac{1}{2}\left\{\sum_{j=1}^{n_{i}} \boldsymbol{x}_{i j} \exp \left(\boldsymbol{x}_{i j}^{\prime} \boldsymbol{\beta}\right) H_{0}\left(t_{i j}\right)\right\}-\frac{1}{2} \sum_{j=1}^{n_{i}} \boldsymbol{x}_{i j} \exp \left(\boldsymbol{x}_{i j}^{\prime} \boldsymbol{\beta}\right) H_{0}\left(t_{i j}\right) \\
& =-\left\{\sum_{j=1}^{n_{i}} \boldsymbol{x}_{i j} \exp \left(\boldsymbol{x}_{i j}^{\prime} \boldsymbol{\beta}\right) H_{0}\left(t_{i j}\right)\right\}\left\{\sum_{j=1}^{n_{i}} \delta_{i j}-\sum_{j=1}^{n_{i}} \exp \left(\boldsymbol{x}_{i j}^{\prime} \boldsymbol{\beta}\right) H_{0}\left(t_{i j}\right)+\frac{1}{2}\right\},
\end{aligned}
$$

$$
\begin{aligned}
& \left.\frac{\partial^{2} \log L_{i}\left(\boldsymbol{\gamma}, \sigma_{u}^{2}\right)}{\partial \sigma_{u}^{2} \partial \boldsymbol{\theta}^{\prime}}\right|_{\sigma_{u}^{2}=0} \\
& =\frac{1}{2}\left[2 \sum_{j=1}^{n_{i}}\left\{\delta_{i j}-H_{0}\left(t_{i j}\right) \exp \left(\boldsymbol{x}_{i j}^{\prime} \boldsymbol{\beta}\right)\right\}-\left\{\sum_{j=1}^{n_{i}} \frac{\partial H_{0}\left(t_{i j}\right)}{\partial \boldsymbol{\theta}} \exp \left(\boldsymbol{x}_{i j}^{\prime} \boldsymbol{\beta}\right)\right\}-\left\{\sum_{j=1}^{n_{i}} \frac{\partial H_{0}\left(t_{i j}\right)}{\partial \boldsymbol{\theta}} \exp \left(\boldsymbol{x}_{i j}^{\prime} \boldsymbol{\beta}\right)\right\}\right] \\
& =\left[\sum_{j=1}^{n_{i}}\left\{\delta_{i j}-H_{0}\left(t_{i j}\right) \exp \left(\boldsymbol{x}_{i j}^{\prime} \boldsymbol{\beta}\right)\right\}-\frac{1}{2}\left\{\sum_{j=1}^{n_{i}} \frac{\partial H_{0}\left(t_{i j}\right)}{\partial \boldsymbol{\theta}} \exp \left(\boldsymbol{x}_{i j}^{\prime} \boldsymbol{\beta}\right)\right\}-\frac{1}{2}\left\{\sum_{j=1}^{n_{i}} \frac{\partial H_{0}\left(t_{i j}\right)}{\partial \boldsymbol{\theta}} \exp \left(\boldsymbol{x}_{i j}^{\prime} \boldsymbol{\beta}\right)\right\}\right] \\
& =-\left\{\sum_{j=1}^{n_{i}} \frac{\partial H_{0}\left(t_{i j}\right)}{\partial \boldsymbol{\theta}} \exp \left(\boldsymbol{x}_{i j}^{\prime} \boldsymbol{\beta}\right)\right\}\left\{\sum_{j=1}^{n_{i}} \delta_{i j}-\sum_{j=1}^{n_{i}} H_{0}\left(t_{i j}\right) \exp \left(\boldsymbol{x}_{i j}^{\prime} \boldsymbol{\beta}\right)+\frac{1}{2}\right\},
\end{aligned}
$$




$$
\begin{aligned}
& \left.\frac{\partial^{2} \log L_{i}\left(\boldsymbol{\gamma}, \sigma_{u}^{2}\right)}{\partial\left(\sigma_{u}^{2}\right)^{2}}\right|_{\sigma_{u}^{2}=0} \\
& =\left.\frac{\partial}{\partial \sigma_{u}^{2}}\left\{\frac{\partial \log L_{i}\left(\boldsymbol{\gamma}, \sigma_{u}^{2}\right)}{\partial \sigma_{u}^{2}}\right\}\right|_{\sigma_{u}^{2}=0} \\
& =\left.\frac{\partial}{\partial \sigma_{u}^{2}}\left\{\frac{1}{L_{i}\left(\boldsymbol{\gamma}, \sigma_{u}^{2}\right)} \frac{\partial}{\partial \sigma_{u}^{2}} L_{i}\left(\boldsymbol{\gamma}, \sigma_{u}^{2}\right)\right\}\right|_{\sigma_{u}^{2}=0} \\
& =\frac{\partial}{\partial \sigma_{u}^{2}}\left\{\frac{1}{L_{i}\left(\gamma, \sigma_{u}^{2}\right)} \frac{\partial}{\partial \sigma_{u}^{2}}\left[\left.\frac{a_{2}}{2 !} \frac{\partial^{2}}{\partial u_{i}^{2}} g^{*}\left(t_{i} \mid u_{i}\right)\right|_{u_{i}=0}+\left.\frac{a_{4}}{4 !} \frac{\partial^{4}}{\partial u_{i}{ }^{4}} g^{*}\left(t_{i} \mid u_{i}\right)\right|_{u_{i}=0}\right]\right\} \\
& =\frac{\partial}{\partial \sigma_{u}^{2}}\left\{\frac{1}{L_{i}\left(\boldsymbol{\gamma}, \sigma_{u}^{2}\right)}\left[\left.\frac{1}{2} \frac{\partial^{2}}{\partial u_{i}^{2}} g^{*}\left(t_{i} \mid u_{i}\right)\right|_{u_{i}=0}+\left.\frac{3 \sigma^{4}}{24} \frac{\partial^{4}}{\partial u_{i}{ }^{4}} g^{*}\left(t_{i} \mid u_{i}\right)\right|_{u_{i}=0}\right]\right\} \\
& =\frac{\partial}{\partial \sigma_{u}^{2}}\left\{\frac{1}{L_{i}\left(\boldsymbol{\gamma}, \sigma_{u}^{2}\right)}\left[\left.\frac{1}{2} \frac{\partial^{2}}{\partial u_{i}{ }^{2}} g^{*}\left(t_{i} \mid u_{i}\right)\right|_{u_{i}=0}\right]+\frac{1}{L_{i}\left(\boldsymbol{\gamma}, \sigma_{u}^{2}\right)}\left[\left.\frac{3\left(\sigma^{2}\right)^{2}}{24} \frac{\partial^{4}}{\partial u_{i}{ }^{4}} g^{*}\left(t_{i} \mid u_{i}\right)\right|_{u_{i}=0}\right]\right\} \\
& =\left\{\frac{\partial}{\partial \sigma_{u}^{2}} \frac{1}{L_{i}\left(\boldsymbol{\gamma}, \sigma_{u}^{2}\right)}\right\}\left\{\frac{1}{2} \frac{\partial^{2}}{\partial u_{i}{ }^{2}} g^{*}\left(t_{i} \mid u_{i}\right)\right\}+\left\{\frac{1}{L_{i}\left(\boldsymbol{\gamma}, \sigma_{u}^{2}\right)}\right\}\left\{\frac{\partial}{\partial \sigma_{u}^{2}}\left[\frac{1}{2} \frac{\partial^{2}}{\partial u_{i}{ }^{2}} g^{*}\left(t_{i} \mid u_{i}\right)\right]\right\} \\
& +\left.\left\{\left[\left\{\frac{\partial}{\partial \sigma_{u}^{2}} \frac{1}{L_{i}\left(\boldsymbol{\gamma}, \sigma_{u}^{2}\right)}\right\}\left\{\frac{\left(\sigma_{u}^{2}\right)^{2}}{8}\right\}+\left\{\frac{1}{L_{i}\left(\boldsymbol{\gamma}, \sigma_{u}^{2}\right)}\right\}\left\{\frac{\partial}{\partial \sigma_{u}^{2}} \frac{\sigma_{u}^{2}}{4}\right\}\right] \frac{\partial^{4}}{\partial u_{i}{ }^{4}} g^{*}\left(t_{i} \mid u_{i}\right)\right\}\right|_{u_{i}=0} \\
& =\left.\left\{\left\{\frac{\partial}{\partial \sigma_{u}^{2}} \frac{1}{L_{i}\left(\boldsymbol{\gamma}, \sigma_{u}^{2}\right)}\right\}\left\{\frac{1}{2} \frac{\partial^{2}}{\partial u_{i}{ }^{2}} g^{*}\left(t_{i} \mid u_{i}\right)\right\}+0+\left\{0+\frac{1}{L_{i}\left(\boldsymbol{\gamma}, \sigma_{u}^{2}\right)} \frac{1}{4} \frac{\partial^{4}}{\partial u_{i}{ }^{4}} g^{*}\left(t_{i} \mid u_{i}\right)\right\}\right\}\right|_{u_{i}=0} \\
& =\left.\left\{\frac{\partial}{\partial \sigma_{u}^{2}} \frac{1}{L_{i}\left(\boldsymbol{\gamma}, \sigma_{u}^{2}\right)}\right\}\left\{\frac{1}{2} \frac{\partial^{2}}{\partial u_{i}{ }^{2}} g^{*}\left(t_{i} \mid u_{i}\right)\right\}\right|_{u_{i}=0}+\left.\left\{\frac{1}{L_{i}\left(\boldsymbol{\gamma}, \sigma_{u}^{2}\right)} \frac{1}{4} \frac{\partial^{4}}{\partial u_{i}{ }^{4}} g^{*}\left(t_{i} \mid u_{i}\right)\right\}\right|_{u_{i}=0}(8.1
\end{aligned}
$$


where

$$
\begin{aligned}
& \left\{\frac{\partial}{\partial \sigma_{u}^{2}} \frac{1}{L_{i}\left(\boldsymbol{\gamma}, \sigma_{u}^{2}\right)}\right\}\left\{\frac{1}{2} \frac{\partial^{2}}{\partial u_{i}{ }^{2}} g^{*}\left(t_{i} \mid u_{i}\right)\right\} \\
& =\left\{-\frac{1}{\left(L_{i}\left(\boldsymbol{\gamma}, \sigma_{u}^{2}\right)\right)^{2}} \frac{\partial\left(L_{i}\left(\boldsymbol{\gamma}, \sigma_{u}^{2}\right)\right.}{\partial \sigma_{u}^{2}}\right\}\left\{\frac{1}{2} \frac{\partial^{2}}{\partial u_{i}^{2}} g^{*}\left(t_{i} \mid u_{i}\right)\right\} \\
& =\left\{-\frac{1}{\left(L_{i}\left(\boldsymbol{\gamma}, \sigma_{u}^{2}\right)\right)^{2}} \frac{1}{2} \frac{\partial^{2}}{\partial u_{i}{ }^{2}} g^{*}\left(t_{i} \mid u_{i}\right)\right\}\left\{\frac{1}{2} \frac{\partial^{2}}{\partial u_{i}{ }^{2}} g^{*}\left(t_{i} \mid u_{i}\right)\right\} \\
& =-\frac{1}{\left(L_{i}\left(\boldsymbol{\gamma}, \sigma_{u}^{2}\right)\right)^{2}}\left\{\frac{1}{2} \frac{\partial^{2}}{\partial u_{i}{ }^{2}} g^{*}\left(t_{i} \mid u_{i}\right)\right\}^{2} \\
& =-\frac{1}{4}\left\{\frac{1}{L_{i}\left(\boldsymbol{\gamma}, \sigma_{u}^{2}\right)} \frac{\partial^{2}}{\partial u_{i}{ }^{2}} g^{*}\left(t_{i} \mid u_{i}\right)\right\}^{2} \\
& =-\frac{1}{4}\left[\left\{\left.\frac{\partial}{\partial u_{i}} \log g^{*}\left(t_{i} \mid u_{i}\right)\right|_{u_{i}=0}\right\}^{2}+\left.\frac{\partial^{2}}{\partial u_{i}{ }^{2}} \log g^{*}\left(t_{i} \mid u_{i}\right)\right|_{u_{i}=0}\right]^{2} \\
& =-\frac{1}{4}\left[\left\{\delta_{i j}-\exp \left(\boldsymbol{x}_{i j}^{\prime} \boldsymbol{\beta}\right) H_{0}\left(t_{i j}\right)\right\}^{2}-\exp \left(\boldsymbol{x}_{i j}^{\prime} \boldsymbol{\beta}\right) H_{0}\left(t_{i j}\right)\right]^{2} \\
& =-\frac{1}{4}\left[\left\{\delta_{i j}-H_{i j}\left(t_{i j}\right)\right\}^{2}-H_{i j}\left(t_{i j}\right)\right]^{2} \\
& =-\frac{1}{4}\left[\left\{\delta_{i j}-H_{i j}\left(t_{i j}\right)\right\}^{4}-2\left\{\delta_{i j}-H_{i j}\left(t_{i j}\right)\right\}^{2} H_{i j}\left(t_{i j}\right)+\left\{H_{i j}\left(t_{i j}\right)\right\}^{2}\right] \\
& =-\frac{1}{4}\left\{\delta_{i j}-H_{i j}\left(t_{i j}\right)\right\}^{4}+\frac{1}{2}\left\{\delta_{i j}-H_{i j}\left(t_{i j}\right)\right\}^{2} H_{i j}\left(t_{i j}\right)-\frac{1}{4}\left\{H_{i j}\left(t_{i j}\right)\right\}^{2},
\end{aligned}
$$




$$
\begin{aligned}
& \frac{1}{L_{i}\left(\boldsymbol{\gamma}, \sigma_{u}^{2}\right)} \frac{1}{4} \frac{\partial^{4}}{\partial u_{i}{ }^{4}} g^{*}\left(t_{i} \mid u_{i}\right) \\
& =\left.\frac{1}{L_{i}\left(\boldsymbol{\gamma}, \sigma_{u}^{2}\right)} \frac{1}{4}\left[\frac{\partial^{2}}{\partial u_{i}}\left\{\frac{\partial^{2}}{\partial u_{i}} g^{*}\left(t_{i} \mid u_{i}\right)\right\}\right]\right|_{u_{i}=0} \\
& =\left.\frac{1}{L_{i}\left(\boldsymbol{\gamma}, \sigma_{u}^{2}\right)} \frac{1}{4}\left[\frac{\partial^{2}}{\partial u_{i}}\left\{g^{*}\left(t_{i} \mid u_{i}\right)\left\{\frac{\partial}{\partial u_{i}} \log g^{*}\left(t_{i} \mid u_{i}\right)\right\}^{2}+g^{*}\left(t_{i} \mid u_{i}\right) \frac{\partial^{2}}{\partial u_{i}{ }^{2}} \log g^{*}\left(t_{i} \mid u_{i}\right)\right\}\right]\right|_{u_{i}=0} \\
& =\left.\frac{1}{L_{i}\left(\boldsymbol{\gamma}, \sigma_{u}^{2}\right)} \frac{1}{4}\left[\frac{\partial}{\partial u_{i}}\left\{\frac{\partial}{\partial u_{i}}\left\{g^{*}\left(t_{i} \mid u_{i}\right)\left\{\frac{\partial}{\partial u_{i}} \log g^{*}\left(t_{i} \mid u_{i}\right)\right\}^{2}+g^{*}\left(t_{i} \mid u_{i}\right) \frac{\partial^{2}}{\partial u_{i}{ }^{2}} \log g^{*}\left(t_{i} \mid u_{i}\right)\right\}\right\}\right]\right|_{u_{i}=0} \\
& =\frac{1}{L_{i}\left(\boldsymbol{\gamma}, \sigma_{u}^{2}\right)} \frac{1}{4}\left[\frac { \partial } { \partial u _ { i } } \left[\frac{\partial}{\partial u_{i}} g^{*}\left(t_{i} \mid u_{i}\right)\left\{\frac{\partial}{\partial u_{i}} \log g^{*}\left(t_{i} \mid u_{i}\right)\right\}^{2}+g^{*}\left(t_{i} \mid u_{i}\right) 2\left\{\frac{\partial}{\partial u_{i}} \log g^{*}\left(t_{i} \mid u_{i}\right)\right\}\right.\right. \\
& \left\{\frac{\partial^{2}}{\partial u_{i}{ }^{2}} \log g^{*}\left(t_{i} \mid u_{i}\right)\right\}+\frac{\partial}{\partial u_{i}} g^{*}\left(t_{i} \mid u_{i}\right)\left\{\frac{\partial^{2}}{\partial u_{i}{ }^{2}} \log g^{*}\left(t_{i} \mid u_{i}\right)\right\}+g^{*}\left(t_{i} \mid u_{i}\right) \\
& \left.\left.\left\{\frac{\partial^{3}}{\partial u_{i}{ }^{3}} \log g^{*}\left(t_{i} \mid u_{i}\right)\right\}\right]\right]\left.\right|_{u_{i}=0} \\
& =\frac{1}{L_{i}\left(\boldsymbol{\gamma}, \sigma_{u}^{2}\right)} \frac{1}{4}\left[\frac { \partial } { \partial u _ { i } } \left[g^{*}\left(t_{i} \mid u_{i}\right)\left\{\frac{\partial}{\partial u_{i}} \log g^{*}\left(t_{i} \mid u_{i}\right)\right\}\left\{\frac{\partial}{\partial u_{i}} \log g^{*}\left(t_{i} \mid u_{i}\right)\right\}^{2}+g^{*}\left(t_{i} \mid u_{i}\right)\right.\right. \\
& 2\left\{\frac{\partial}{\partial u_{i}} \log g^{*}\left(t_{i} \mid u_{i}\right)\right\}\left\{\frac{\partial^{2}}{\partial u_{i}{ }^{2}} \log g^{*}\left(t_{i} \mid u_{i}\right)\right\}+g^{*}\left(t_{i} \mid u_{i}\right)\left\{\frac{\partial}{\partial u_{i}} \log g^{*}\left(t_{i} \mid u_{i}\right)\right\} \\
& \left.\left\{\frac{\partial^{2}}{\partial u_{i}{ }^{2}} \log g^{*}\left(t_{i} \mid u_{i}\right)\right\}+g^{*}\left(t_{i} \mid u_{i}\right)\left\{\frac{\partial^{3}}{\partial u_{i}{ }^{3}} \log g^{*}\left(t_{i} \mid u_{i}\right)\right\}\right]\left.\right|_{u_{i}=0} \\
& =\frac{1}{L_{i}\left(\gamma, \sigma_{u}^{2}\right)} \frac{1}{4}\left[\frac { \partial } { \partial u _ { i } } \left[g^{*}\left(t_{i} \mid u_{i}\right)\left\{\frac{\partial}{\partial u_{i}} \log g^{*}\left(t_{i} \mid u_{i}\right)\right\}^{3}+g^{*}\left(t_{i} \mid u_{i}\right) 2\left\{\frac{\partial}{\partial u_{i}} \log g^{*}\left(t_{i} \mid u_{i}\right)\right\}\right.\right. \\
& \left\{\frac{\partial^{2}}{\partial u_{i}{ }^{2}} \log g^{*}\left(t_{i} \mid u_{i}\right)\right\}+g^{*}\left(t_{i} \mid u_{i}\right)\left\{\frac{\partial}{\partial u_{i}} \log g^{*}\left(t_{i} \mid u_{i}\right)\right\}\left\{\frac{\partial^{2}}{\partial u_{i}{ }^{2}} \log g^{*}\left(t_{i} \mid u_{i}\right)\right\} \\
& \left.\left.+g^{*}\left(t_{i} \mid u_{i}\right)\left\{\frac{\partial^{3}}{\partial u_{i}{ }^{3}} \log g^{*}\left(t_{i} \mid u_{i}\right)\right\}\right]\right]\left.\right|_{u_{i}=0}
\end{aligned}
$$




$$
\begin{aligned}
& =\frac{1}{L_{i}\left(\boldsymbol{\gamma}, \sigma_{u}^{2}\right)} \frac{1}{4}\left[\frac{\partial}{\partial u_{i}} g^{*}\left(t_{i} \mid u_{i}\right)\left\{\frac{\partial}{\partial u_{i}} \log g^{*}\left(t_{i} \mid u_{i}\right)\right\}^{3}+g^{*}\left(t_{i} \mid u_{i}\right) 3\left\{\frac{\partial}{\partial u_{i}} \log g^{*}\left(t_{i} \mid u_{i}\right)\right\}^{2}\right. \\
& \left\{\frac{\partial^{2}}{\partial u_{i}{ }^{2}} \log g^{*}\left(t_{i} \mid u_{i}\right)\right\}+\left\{\frac{\partial}{\partial u_{i}} \log g^{*}\left(t_{i} \mid u_{i}\right)\right\} 2\left\{\frac{\partial}{\partial u_{i}} \log g^{*}\left(t_{i} \mid u_{i}\right)\right\}\left\{\frac{\partial^{2}}{\partial u_{i}{ }^{2}} \log g^{*}\left(t_{i} \mid u_{i}\right)\right\} \\
& +g^{*}\left(t_{i} \mid u_{i}\right) 2\left\{\frac{\partial^{2}}{\partial u_{i}{ }^{2}} \log g^{*}\left(t_{i} \mid u_{i}\right)\right\}\left\{\frac{\partial^{2}}{\partial u_{i}{ }^{2}} \log g^{*}\left(t_{i} \mid u_{i}\right)\right\}+g^{*}\left(t_{i} \mid u_{i}\right) 2\left\{\frac{\partial}{\partial u_{i}} \log g^{*}\left(t_{i} \mid u_{i}\right)\right\} \\
& \left\{\frac{\partial^{3}}{\partial u_{i}{ }^{3}} \log g^{*}\left(t_{i} \mid u_{i}\right)\right\}+\left\{\frac{\partial}{\partial u_{i}} \log g^{*}\left(t_{i} \mid u_{i}\right)\right\}\left\{\frac{\partial}{\partial u_{i}} \log g^{*}\left(t_{i} \mid u_{i}\right)\right\}\left\{\frac{\partial^{2}}{\partial u_{i}{ }^{2}} \log g^{*}\left(t_{i} \mid u_{i}\right)\right\} \\
& +g^{*}\left(t_{i} \mid u_{i}\right)\left\{\frac{\partial^{2}}{\partial u_{i}{ }^{2}} \log g^{*}\left(t_{i} \mid u_{i}\right)\right\}\left\{\frac{\partial^{2}}{\partial u_{i}{ }^{2}} \log g^{*}\left(t_{i} \mid u_{i}\right)\right\}+g^{*}\left(t_{i} \mid u_{i}\right)\left\{\frac{\partial}{\partial u_{i}} \log g^{*}\left(t_{i} \mid u_{i}\right)\right\} \\
& \left\{\frac{\partial^{3}}{\partial u_{i}{ }^{3}} \log g^{*}\left(t_{i} \mid u_{i}\right)\right\}+\left\{\frac{\partial}{\partial u_{i}} \log g^{*}\left(t_{i} \mid u_{i}\right)\right\}\left\{\frac{\partial^{3}}{\partial u_{i}{ }^{3}} g^{*}\left(t_{i} \mid u_{i}\right)\right\}+g^{*}\left(t_{i} \mid u_{i}\right) \\
& \left.\left\{\frac{\partial^{4}}{\partial u_{i}{ }^{4}} \log g^{*}\left(t_{i} \mid u_{i}\right)\right\}\right]\left.\right|_{u_{i}=0} \\
& =\frac{1}{L_{i}\left(\boldsymbol{\gamma}, \sigma_{u}^{2}\right)} \frac{1}{4}\left[g^{*}\left(t_{i} \mid u_{i}\right)\left\{\frac{\partial}{\partial u_{i}} \log g^{*}\left(t_{i} \mid u_{i}\right)\right\}\left\{\frac{\partial}{\partial u_{i}} \log g^{*}\left(t_{i} \mid u_{i}\right)\right\}^{3}+g^{*}\left(t_{i} \mid u_{i}\right)\right. \\
& 3\left\{\frac{\partial}{\partial u_{i}} \log g^{*}\left(t_{i} \mid u_{i}\right)\right\}^{2}\left\{\frac{\partial^{2}}{\partial u_{i}{ }^{2}} \log g^{*}\left(t_{i} \mid u_{i}\right)\right\}+g^{*}\left(t_{i} \mid u_{i}\right)\left\{\frac{\partial}{\partial u_{i}} \log g^{*}\left(t_{i} \mid u_{i}\right)\right\} \\
& 2\left\{\frac{\partial}{\partial u_{i}} \log g^{*}\left(t_{i} \mid u_{i}\right)\right\}\left\{\frac{\partial^{2}}{\partial u_{i}{ }^{2}} \log g^{*}\left(t_{i} \mid u_{i}\right)\right\}+g^{*}\left(t_{i} \mid u_{i}\right) 2\left\{\frac{\partial^{2}}{\partial u_{i}{ }^{2}} \log g^{*}\left(t_{i} \mid u_{i}\right)\right\} \\
& \left\{\frac{\partial^{2}}{\partial u_{i}{ }^{2}} \log g^{*}\left(t_{i} \mid u_{i}\right)\right\}+g^{*}\left(t_{i} \mid u_{i}\right) 2\left\{\frac{\partial}{\partial u_{i}} \log g^{*}\left(t_{i} \mid u_{i}\right)\right\}\left\{\frac{\partial^{3}}{\partial u_{i}{ }^{3}} \log g^{*}\left(t_{i} \mid u_{i}\right)\right\} \\
& +g^{*}\left(t_{i} \mid u_{i}\right)\left\{\frac{\partial}{\partial u_{i}} \log g^{*}\left(t_{i} \mid u_{i}\right)\right\}\left\{\frac{\partial}{\partial u_{i}} \log g^{*}\left(t_{i} \mid u_{i}\right)\right\}\left\{\frac{\partial^{2}}{\partial u_{i}{ }^{2}} \log g^{*}\left(t_{i} \mid u_{i}\right)\right\} \\
& +g^{*}\left(t_{i} \mid u_{i}\right)\left\{\frac{\partial^{2}}{\partial u_{i}{ }^{2}} \log g^{*}\left(t_{i} \mid u_{i}\right)\right\}\left\{\frac{\partial^{2}}{\partial u_{i}{ }^{2}} \log g^{*}\left(t_{i} \mid u_{i}\right)\right\}+g^{*}\left(t_{i} \mid u_{i}\right)\left\{\frac{\partial}{\partial u_{i}} \log g^{*}\left(t_{i} \mid u_{i}\right)\right\} \\
& \left\{\frac{\partial^{3}}{\partial u_{i}{ }^{3}} \log g^{*}\left(t_{i} \mid u_{i}\right)\right\}+g^{*}\left(t_{i} \mid u_{i}\right)\left\{\frac{\partial}{\partial u_{i}} \log g^{*}\left(t_{i} \mid u_{i}\right)\right\}\left\{\frac{\partial^{3}}{\partial u_{i}{ }^{3}} \log g^{*}\left(t_{i} \mid u_{i}\right)\right\} \\
& \left.+g^{*}\left(t_{i} \mid u_{i}\right)\left\{\frac{\partial^{4}}{\partial u_{i}{ }^{4}} \log g^{*}\left(t_{i} \mid u_{i}\right)\right\}\right]\left.\right|_{u_{i}=0}
\end{aligned}
$$




$$
\begin{aligned}
& =\frac{1}{g^{*}\left(t_{i} \mid u_{i}\right)} \frac{1}{4}\left[g^{*}\left(t_{i} \mid u_{i}\right)\left\{\frac{\partial}{\partial u_{i}} \log g^{*}\left(t_{i} \mid u_{i}\right)\right\}^{4}+g^{*}\left(t_{i} \mid u_{i}\right) 3\left\{\frac{\partial}{\partial u_{i}} \log g^{*}\left(t_{i} \mid u_{i}\right)\right\}^{2}\right. \\
& \left\{\frac{\partial^{2}}{\partial u_{i}{ }^{2}} \log g^{*}\left(t_{i} \mid u_{i}\right)\right\}+g^{*}\left(t_{i} \mid u_{i}\right) 2\left\{\frac{\partial}{\partial u_{i}} \log g^{*}\left(t_{i} \mid u_{i}\right)\right\}^{2}\left\{\frac{\partial^{2}}{\partial u_{i}{ }^{2}} \log g^{*}\left(t_{i} \mid u_{i}\right)\right\} \\
& +g^{*}\left(t_{i} \mid u_{i}\right) 2\left\{\frac{\partial^{2}}{\partial u_{i}{ }^{2}} \log g^{*}\left(t_{i} \mid u_{i}\right)\right\}^{2}+g^{*}\left(t_{i} \mid u_{i}\right) 2\left\{\frac{\partial}{\partial u_{i}} \log g^{*}\left(t_{i} \mid u_{i}\right)\right\} \\
& \left\{\frac{\partial^{3}}{\partial u_{i}{ }^{3}} \log g^{*}\left(t_{i} \mid u_{i}\right)\right\}+g^{*}\left(t_{i} \mid u_{i}\right)\left\{\frac{\partial}{\partial u_{i}} \log g^{*}\left(t_{i} \mid u_{i}\right)\right\}^{2}\left\{\frac{\partial^{2}}{\partial u_{i}{ }^{2}} \log g^{*}\left(t_{i} \mid u_{i}\right)\right\}+g^{*}\left(t_{i} \mid u_{i}\right) \\
& \left\{\frac{\partial^{2}}{\partial u_{i}{ }^{2}} \log g^{*}\left(t_{i} \mid u_{i}\right)\right\}^{2}+g^{*}\left(t_{i} \mid u_{i}\right)\left\{\frac{\partial}{\partial u_{i}} \log g^{*}\left(t_{i} \mid u_{i}\right)\right\}\left\{\frac{\partial^{3}}{\partial u_{i}{ }^{3}} \log g^{*}\left(t_{i} \mid u_{i}\right)\right\}+g^{*}\left(t_{i} \mid u_{i}\right) \\
& \left.\left\{\frac{\partial}{\partial u_{i}} \log g^{*}\left(t_{i} \mid u_{i}\right)\right\}\left\{\frac{\partial^{3}}{\partial u_{i}{ }^{3}} \log g^{*}\left(t_{i} \mid u_{i}\right)\right\}+g^{*}\left(t_{i} \mid u_{i}\right)\left\{\frac{\partial^{4}}{\partial u_{i}{ }^{4}} \log g^{*}\left(t_{i} \mid u_{i}\right)\right\}\right]\left.\right|_{u_{i}=0} \\
& =\frac{1}{4}\left[\left\{\frac{\partial}{\partial u_{i}} \log g^{*}\left(t_{i} \mid u_{i}\right)\right\}^{4}+3\left\{\frac{\partial}{\partial u_{i}} \log g^{*}\left(t_{i} \mid u_{i}\right)\right\}^{2}\left\{\frac{\partial^{2}}{\partial u_{i}{ }^{2}} \log g^{*}\left(t_{i} \mid u_{i}\right)\right\}\right. \\
& +2\left\{\frac{\partial}{\partial u_{i}} \log g^{*}\left(t_{i} \mid u_{i}\right)\right\}^{2}\left\{\frac{\partial^{2}}{\partial u_{i}{ }^{2}} \log g^{*}\left(t_{i} \mid u_{i}\right)\right\}+2\left\{\frac{\partial^{2}}{\partial u_{i}{ }^{2}} \log g^{*}\left(t_{i} \mid u_{i}\right)\right\}^{2} \\
& +2\left\{\frac{\partial}{\partial u_{i}} \log g^{*}\left(t_{i} \mid u_{i}\right)\right\}\left\{\frac{\partial^{3}}{\partial u_{i}{ }^{3}} \log g^{*}\left(t_{i} \mid u_{i}\right)\right\}+\left\{\frac{\partial}{\partial u_{i}} \log g^{*}\left(t_{i} \mid u_{i}\right)\right\}^{2}\left\{\frac{\partial^{2}}{\partial u_{i}{ }^{2}} \log g^{*}\left(t_{i} \mid u_{i}\right)\right\} \\
& +\left\{\frac{\partial^{2}}{\partial u_{i}{ }^{2}} \log g^{*}\left(t_{i} \mid u_{i}\right)\right\}^{2}+\left\{\frac{\partial}{\partial u_{i}} \log g^{*}\left(t_{i} \mid u_{i}\right)\right\}\left\{\frac{\partial^{3}}{\partial u_{i}{ }^{3}} \log g^{*}\left(t_{i} \mid u_{i}\right)\right\}+\left\{\frac{\partial}{\partial u_{i}} \log g^{*}\left(t_{i} \mid u_{i}\right)\right\} \\
& \left.\left\{\frac{\partial^{3}}{\partial u_{i}{ }^{3}} \log g^{*}\left(t_{i} \mid u_{i}\right)\right\}+\left\{\frac{\partial^{4}}{\partial u_{i}{ }^{4}} \log g^{*}\left(t_{i} \mid u_{i}\right)\right\}^{2}\right]\left.\right|_{u_{i}=0} \\
& =\frac{1}{4}\left[\left\{\delta_{i j}-H_{i j}\left(t_{i j}\right)\right\}^{4}+3\left\{\delta_{i j}-H_{i j}\left(t_{i j}\right)\right\}^{2}\left\{-H_{i j}\left(t_{i j}\right)\right\}+2\left\{\delta_{i j}-H_{i j}\left(t_{i j}\right)\right\}^{2}\left\{-H_{i j}\left(t_{i j}\right)\right\}\right. \\
& +2\left\{-H_{i j}\left(t_{i j}\right)\right\}^{2}+2\left\{\delta_{i j}-H_{i j}\left(t_{i j}\right)\right\}\left\{-H_{i j}\left(t_{i j}\right)\right\}+\left\{\delta_{i j}-H_{i j}\left(t_{i j}\right)\right\}^{2}\left\{-H_{i j}\left(t_{i j}\right)\right\} \\
& \left.+\left\{-H_{i j}\left(t_{i j}\right)\right\}^{2}+\left\{\delta_{i j}-H_{i j}\left(t_{i j}\right)\right\}\left\{-H_{i j}\left(t_{i j}\right)\right\}+\left\{\delta_{i j}-H_{i j}\left(t_{i j}\right)\right\}\left\{-H_{i j}\left(t_{i j}\right)\right\}-\left\{H_{i j}\left(t_{i j}\right)\right\}\right] \\
& =\frac{1}{4}\left[\left\{\delta_{i j}-H_{i j}\left(t_{i j}\right)\right\}^{4}-3\left\{\delta_{i j}-H_{i j}\left(t_{i j}\right)\right\}^{2}\left\{H_{i j}\left(t_{i j}\right)\right\}-2\left\{\delta_{i j}-H_{i j}\left(t_{i j}\right)\right\}^{2}\left\{H_{i j}\left(t_{i j}\right)\right\}\right. \\
& +2\left\{H_{i j}\left(t_{i j}\right)\right\}^{2}-2\left\{\delta_{i j}-H_{i j}\left(t_{i j}\right)\right\}\left\{H_{i j}\left(t_{i j}\right)\right\}-\left\{\delta_{i j}-H_{i j}\left(t_{i j}\right)\right\}^{2}\left\{H_{i j}\left(t_{i j}\right)\right\}+\left\{H_{i j}\left(t_{i j}\right)\right\}^{2} \\
& \left.-\left\{\delta_{i j}-H_{i j}\left(t_{i j}\right)\right\}\left\{H_{i j}\left(t_{i j}\right)\right\}-\left\{\delta_{i j}-H_{i j}\left(t_{i j}\right)\right\}\left\{H_{i j}\left(t_{i j}\right)\right\}-\left\{H_{i j}\left(t_{i j}\right)\right\}\right]
\end{aligned}
$$




$$
\begin{aligned}
= & \left.\left.\left.\frac{1}{4}\left[\left\{\delta_{i j}-H_{(} t_{i j}\right)\right\}^{4}-6\left\{\delta_{i j}-H_{(} t_{i j}\right)\right\}^{2}\left\{H_{(} t_{i j}\right)\right\}-4\left\{\delta_{i j}-H_{(} t_{i j}\right)\right\}\left\{H_{(}\left(t_{i j}\right)\right\} \\
& \left.\left.+3\left\{H_{(}\left(t_{i j}\right)\right\}^{2}-\left\{H_{(} t_{i j}\right)\right\}\right] \\
= & \left.\left.\left.\frac{1}{4}\left\{\delta_{i j}-H_{(}\left(t_{i j}\right)\right\}^{4}-\frac{3}{2}\left\{\delta_{i j}-H_{(} t_{i j}\right)\right\}^{2}\left\{H_{(} t_{i j}\right)\right\}-\left\{\delta_{i j}-H_{(} t_{i j}\right) H_{i j}\left(t_{i j}\right)\right\} \\
& +\frac{3}{4}\left\{H_{(}\left(t_{i j}\right)\right\}^{2}-\frac{1}{4}\left\{H_{(}\left(t_{i j}\right)\right\}
\end{aligned}
$$

$$
\begin{aligned}
= & -\frac{1}{4}\left\{\delta_{i j}-H_{i j}\left(t_{i j}\right)\right\}^{4}+\frac{1}{2}\left\{\delta_{i j}-H_{i j}\left(t_{i j}\right)\right\}^{2} H_{i j}\left(t_{i j}\right)-\frac{1}{4}\left\{H_{i j}\left(t_{i j}\right)\right\}^{2}+\frac{1}{4}\left\{\delta_{i j}-H_{i j}\left(t_{i j}\right)\right\}^{4} \\
& -\frac{3}{2}\left\{\delta_{i j}-H_{i j}\left(t_{i j}\right)\right\}^{2}\left\{H_{i j}\left(t_{i j}\right)\right\}-\left\{\delta_{i j}-H_{i j}\left(t_{i j}\right)\right\}\left\{H_{i j}\left(t_{i j}\right)\right\}+\frac{3}{4}\left\{H_{i j}\left(t_{i j}\right)\right\}^{2}-\frac{1}{4}\left\{H_{i j}\left(t_{i j}\right)\right\} \\
= & -\left\{\delta_{i j}-H_{i j}\left(t_{i j}\right)\right\}^{2}\left\{H_{i j}\left(t_{i j}\right)\right\}-\left\{\delta_{i j}-H_{i j}\left(t_{i j}\right)\right\}\left\{H_{i j}\left(t_{i j}\right)\right\}+\frac{1}{2}\left\{H_{i j}\left(t_{i j}\right)\right\}^{2}-\frac{1}{4}\left\{H_{i j}\left(t_{i j}\right)\right\} \\
= & -\left\{H_{i j}\left(t_{i j}\right)\right\}\left\{\delta_{i j}-H_{i j}\left(t_{i j}\right)\right\}\left\{\delta_{i j}-H_{i j}\left(t_{i j}\right)+1\right\}+\frac{1}{2}\left\{H_{i j}\left(t_{i j}\right)\right\}^{2}-\frac{1}{4}\left\{H_{i j}\left(t_{i j}\right)\right\} \\
= & -\left\{\exp \left(\boldsymbol{x}_{i j}^{\prime} \boldsymbol{\beta}+u_{i}\right) h_{0}(t)\right\}\left\{\delta_{i j}-\exp \left(\boldsymbol{x}_{i j}^{\prime} \boldsymbol{\beta}+u_{i}\right) h_{0}(t)\right\}\left\{\left[\delta_{i j}-\exp \left(\boldsymbol{x}_{i j}^{\prime} \boldsymbol{\beta}+u_{i}\right) h_{0}(t)\right]+1\right\} \\
& +\frac{1}{2}\left\{\exp \left(\boldsymbol{x}_{i j}^{\prime} \boldsymbol{\beta}+u_{i}\right) h_{0}(t)\right\}^{2}-\frac{1}{4}\left\{\exp \left(\boldsymbol{x}_{i j}^{\prime} \boldsymbol{\beta}+u_{i}\right) h_{0}(t)\right\} .
\end{aligned}
$$

\subsection{Blader Cancer Data}


Table 8.1: Multiple Tumour Recurrence Data for Placebo \& Thiotepa Groups (Wei [30])

\begin{tabular}{|c|c|c|c|c|c|c|c|c|c|c|c|c|}
\hline $\begin{array}{l}\text { Patient } \\
\text { number }\end{array}$ & $\begin{array}{l}\text { Treat } \\
\text { group }\end{array}$ & $\begin{array}{c}\text { Initial } \\
\text { number }\end{array}$ & $\begin{array}{c}\text { Initial } \\
\text { size }\end{array}$ & $\begin{array}{c}\text { ollow-u } \\
\text { time }\end{array}$ & $\begin{array}{c}\text { Recurren } \\
123\end{array}$ & tim & $\begin{array}{l}\mathrm{e} \\
\mathrm{NO} \text { of } \\
\text { recurrences }\end{array}$ & $\begin{array}{l}\text { Survival } \\
\text { status }\end{array}$ & $\begin{array}{c}\mathrm{G} \\
1\end{array}$ & $\begin{array}{c}\mathrm{apt} \\
2 \\
\end{array}$ & $\begin{array}{l}\operatorname{tim} \\
3\end{array}$ & $\begin{array}{r}\mathrm{me} \\
4 \\
\end{array}$ \\
\hline 1 & 1 & 1 & 3 & 1 & & & 0 & 0 & 1 & 0 & 0 & $\overline{0}$ \\
\hline 2 & 1 & 2 & 1 & 4 & & & 0 & 0 & 4 & 0 & 0 & 0 \\
\hline 3 & 1 & 1 & 1 & 7 & & & 0 & 0 & 7 & 0 & 0 & 0 \\
\hline 4 & 1 & 5 & 1 & 10 & & & 0 & 0 & 10 & 0 & 0 & 0 \\
\hline 5 & 1 & 4 & 1 & 10 & 6 & & 1 & 1 & 6 & 4 & 0 & 0 \\
\hline 6 & 1 & 1 & 1 & 14 & & & 0 & 0 & 14 & 0 & 0 & 0 \\
\hline 7 & 1 & 1 & 1 & 18 & & & 0 & 0 & & 0 & 0 & 0 \\
\hline 8 & 1 & 1 & 3 & 18 & 5 & & 1 & 1 & 5 & 13 & 0 & 0 \\
\hline 9 & 1 & 1 & 1 & 18 & 1216 & & 2 & 1 & 12 & 4 & 2 & 0 \\
\hline 10 & 1 & 3 & 3 & 23 & & & 0 & 0 & 23 & 0 & 0 & 0 \\
\hline 11 & 1 & 1 & 3 & 23 & 1015 & & 2 & 1 & 10 & 5 & 8 & 0 \\
\hline 12 & 1 & 1 & 1 & 23 & 316 & & 2 & 1 & & 13 & 7 & 0 \\
\hline 13 & 1 & 3 & 1 & 23 & $\begin{array}{lll}3 & 9 & 21\end{array}$ & & 3 & 1 & 3 & 61 & 12 & 2 \\
\hline 14 & 1 & 2 & 3 & 24 & 71016 & & 3 & 1 & 7 & 3 & 6 & 8 \\
\hline 15 & 1 & 1 & 1 & 25 & 315 & & 2 & 1 & 3 & & 10 & 0 \\
\hline 16 & 1 & 1 & 2 & 26 & & & 0 & 0 & 26 & 0 & 0 & 0 \\
\hline 17 & 1 & 8 & 1 & 26 & 1 & & 1 & 1 & 1 & 25 & 0 & 0 \\
\hline 18 & 1 & 1 & 4 & 26 & 2 & & 1 & 1 & 2 & 24 & 0 & 0 \\
\hline 19 & 1 & 1 & 2 & 28 & 25 & & 1 & 1 & 25 & 3 & 0 & 0 \\
\hline 20 & 1 & 1 & 4 & 29 & & & 0 & 0 & 29 & 0 & 0 & 0 \\
\hline 21 & 1 & 1 & 2 & 29 & & & 0 & 0 & 29 & 0 & 0 & 0 \\
\hline 22 & 1 & 4 & 1 & 29 & & & 0 & 0 & 29 & 0 & 0 & 0 \\
\hline 23 & 1 & 1 & 6 & 30 & 28 & & 1 & 1 & 28 & 2 & 0 & 0 \\
\hline 24 & 1 & 1 & 5 & 30 & 21722 & & 3 & 1 & & 15 & 5 & 8 \\
\hline 25 & 1 & 2 & 1 & 30 & $\begin{array}{lll}3 & 6 & 8\end{array}$ & 12 & 4 & 1 & 3 & 3 & 2 & 4 \\
\hline 26 & 1 & 1 & 3 & 31 & 121524 & & 3 & 1 & 12 & 3 & 9 & 7 \\
\hline 27 & 1 & 1 & 2 & 32 & & & 0 & 0 & 32 & 0 & 0 & 0 \\
\hline 28 & 1 & 2 & 1 & 34 & & & 0 & 0 & 34 & 0 & 0 & 0 \\
\hline 29 & 1 & 2 & 1 & 36 & & & 0 & 0 & 36 & 0 & 0 & 0 \\
\hline 30 & 1 & 3 & 1 & 36 & 29 & & 1 & 1 & 29 & 9 & 0 & 0 \\
\hline 31 & 1 & 1 & 2 & 37 & & & 0 & 0 & 37 & 0 & 0 & 0 \\
\hline 32 & 1 & 4 & 1 & 40 & 91722 & 24 & 4 & 1 & 9 & 8 & 5 & 2 \\
\hline 33 & 1 & 5 & 1 & 40 & 161923 & 29 & 4 & 1 & 16 & 3 & 4 & 6 \\
\hline 34 & 1 & 1 & 2 & 41 & & & 0 & 0 & & 0 & 0 & 0 \\
\hline 35 & 1 & 1 & 1 & 43 & 3 & & 1 & 1 & 3 & 40 & 0 & 0 \\
\hline 36 & 1 & 2 & 6 & 43 & 6 & & 1 & 1 & 6 & 37 & 0 & 0 \\
\hline 37 & 1 & 2 & 1 & 44 & $\begin{array}{lll}3 & 6 & 9\end{array}$ & & 3 & 1 & 3 & 3 & 3 & 35 \\
\hline 38 & 1 & 1 & 1 & 45 & 91120 & 26 & 4 & 1 & 9 & 2 & 9 & 6 \\
\hline 39 & 1 & 1 & 1 & 48 & 18 & & 1 & 1 & & 330 & 0 & 0 \\
\hline 40 & 1 & 1 & 3 & 49 & & & 0 & 0 & & 0 & 0 & 0 \\
\hline 41 & 1 & 3 & 1 & 51 & 35 & & 1 & 1 & & 16 & 0 & 0 \\
\hline 42 & 1 & 1 & 7 & 53 & 17 & & 1 & 1 & & 36 & 0 & 0 \\
\hline 43 & 1 & 3 & 1 & 53 & 31546 & 51 & 4 & 1 & & $12:$ & 31 & 5 \\
\hline
\end{tabular}


Table 8.2: Multiple Tumour Recurrence Data for Placebo \& Thiotepa Groups (Wei [30])

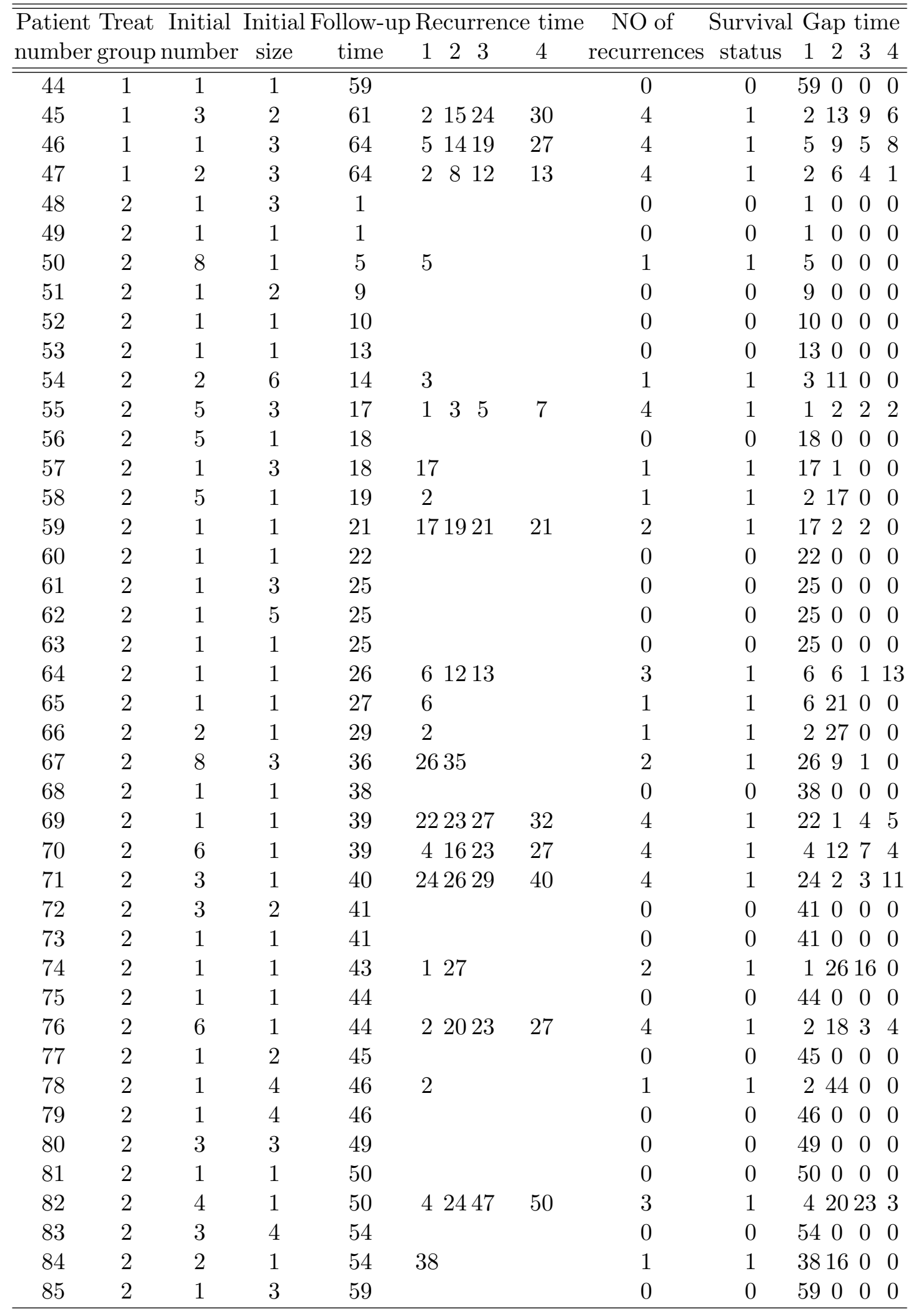




\section{Bibliography}

[1] Aalen O (1978). Nonparametric inference for a family of counting processes. Ann.Statist., 6(4): 701-726, 07. URL http://dx.doi.org/10.1214/aos/1176344247.

[2] Aalen O (1988). Heterogeneity in survival analysis. Statistics in medicine, 7(11): 11211137.

[3] Andrews DF and Herzberg AM (1985). Multiple tumour recurrence data for patients with bladder cancer. In Data, 253-260. Springer.

[4] Blossfeld HP and Hamerle A (1989). Unobserved heterogeneity in hazard rate models: A test and an illustration from a study of career mobility. Quality and Quantity, 23(2): 129-141.

[5] Box-Steffensmeier JM, De Boef S, and Joyce KA (2007). Event dependence and heterogeneity in duration models: the conditional frailty model. Political Analysis, 15(3): 237-256.

[6] Byar DP (1980). The veterans administration study of chemoprophylaxis for recurrent stage I bladder tumours: comparisons of placebo, pyridoxine and topical thiotepa. In Bladder tumors and other topics in urological oncology, 363-370. Springer.

[7] Claeskens G, Nguti R, and Janssen P (2008). One-sided tests in shared frailty models. Test,17(1): 69-82.

[8] Crainiceanu CM and Ruppert D (2004). Likelihood ratio tests in linear mixed models with one variance component. Journal of the Royal Statistical Society: Series B, 66(1):165-185.

[9] Collett D (2015). Modelling survival data in medical research. CRC press. 
[10] Commenges D and Andersen PK (1995). Score test of homogeneity for survival data. Lifetime Data Analysis, 1(2): 145-156.

[11] Cook RJ and Lawless JF (2007). The statistical analysis of recurrent events. Springer Science \& Business Media.

[12] Cox DR (1972). Regression models and life-tables. Journal of the Royal Statistical Society. Series B (Methodological), 34(2): 187-220. URL http://www.jstor.org/stable/2985181.

[13] Duchateau L and Janssen P (2007). The Frailty Model. Springer Science \& Business Media.

[14] Fitzmaurice GM, Lipsitz SR, and Ibrahim JG (2007). A note on permutation tests for variance components in multilevel generalized linear mixed models. Biometrics, 63(3): $942-946$.

[15] Harrington DP and Fleming TR (1982). A class of rank test procedures for censored survival data. Biometrika, 69(3): 553-566.

[16] Harrell F (2001). Regression modelling strategies: with applications to linear models, logistic regression, and survival analysis. Springer-Verlag New York.

[17] Hougaard P (1995). Frailty models for survival data. Lifetime data analysis, 1(3): 255-273.

[18] Hougaard P (2012). Analysis of multivariate survival data. Springer Science \& Business Media.

[19] Kaplan EL and Meier P (1958). Nonparametric estimation from incomplete observations. Journal of the American statistical association, 53(282): 457-481.

[20] Kleinbaum DG and Klein M (2012). Survival analysis: a self-learning text, Third Edition. New York, NY : Springer Science \& Business Media. 
[21] McGilchrist CA and Aisbett CW (1991). Regression with frailty in survival analysis. Biometrics, pages 461- 466 .

[22] Murthy DP, Xie M, and Jiang R (2004). Weibull models, volume 505. John Wiley \& Sons.

[23] Nelson W (1972). Theory and applications of hazard plotting for censored failure data. Technometrics, 14(4): 945-966.

[24] Silvapulle M and Silvapulle P (1995). A score test against one-sided alternatives. Journal of the American Statistical Association, 90(429): 342-349.

[25] Sinha SK (2009). Bootstrap tests for variance components in generalized linear mixed models. Canadian Journal of Statistics, 37(2):219-234.

[26] Sinha SK (2012). The use of score tests for frailty variance components in recurrent event data. Journal of Biometrics $\&$ Biostatistics.

[27] Therneau TM and Grambsch PM (2000). Modeling survival data: extending the Cox model. Springer Science \& Business Media.

[28] Vaida F and Ronghui X (2000). Proportional hazards model with random effects. Statistics in medicine, 19(24):3309-3324.

[29] Verbeke G and Molenberghs G (2003). The use of score tests for inference on variance components. Biometrics, 59(2): 254262.

[30] Wei LJ, Lin DY, and Weissfeld L (1989). Regression analysis of multivariate incomplete failure time data by modeling marginal distributions. Journal of the American statistical association, 84(408):1065-1073.

[31] Wienke A (2011). Frailty Models in Survival Analysis. Chapman and Hall/CRC. 